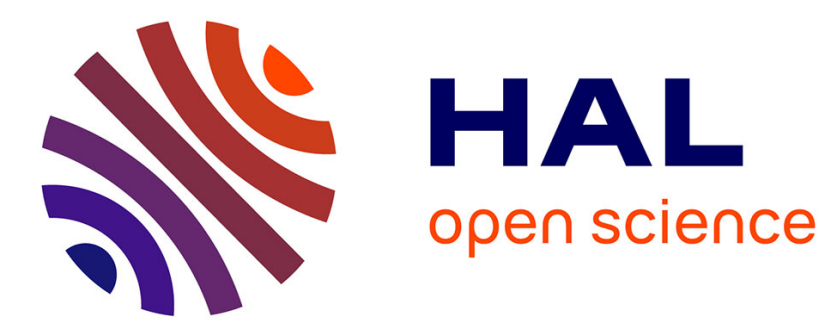

\title{
Loss of Polycomb Protein EZH2 causes major depletion of H3K27 and H3K9 tri-methylation and developmental defects in the fungus Podospora anserina
}

F Carlier, R Debuchy, L Maroc, C Souaid, Daan Noordermeer, P Grognet, F Malagnac

\section{To cite this version:}

F Carlier, R Debuchy, L Maroc, C Souaid, Daan Noordermeer, et al.. Loss of Polycomb Protein EZH2 causes major depletion of H3K27 and H3K9 tri-methylation and developmental defects in the fungus Podospora anserina. 2020. hal-02991015

\section{HAL Id: hal-02991015 https://hal.science/hal-02991015}

Preprint submitted on 5 Nov 2020

HAL is a multi-disciplinary open access archive for the deposit and dissemination of scientific research documents, whether they are published or not. The documents may come from teaching and research institutions in France or abroad, or from public or private research centers.
L'archive ouverte pluridisciplinaire HAL, est destinée au dépôt et à la diffusion de documents scientifiques de niveau recherche, publiés ou non, émanant des établissements d'enseignement et de recherche français ou étrangers, des laboratoires publics ou privés. 
Loss of Polycomb Protein EZH2 causes major depletion of H3K27 and H3K9 trimethylation and developmental defects in the fungus Podospora anserina

Carlier $F^{1,3}$, Debuchy $R^{1}$, Maroc $L^{2}$, Souaid $C^{1,4}$, Noordermeer $D^{1}$, Grognet $P^{1} \&$ Malagnac F'.

1 Université Paris-Saclay, CEA, CNRS, Institute for Integrative Biology of the Cell (I2BC), 91198, Gif-sur-Yvette, France

2 Génétique Quantitative et Évolution-Le Moulon, INRA-Université Paris-Saclay-CNRSAgroParisTech,

Batiment 400, UFR des Sciences, F 91405, Orsay, CEDEX, France.

3 Present address: Group Fungal Epigenomics, Department of Mycology, Institut Pasteur, Paris, France

4 Present address: Aix-Marseille University, Inserm, Theories and Approaches of Genomic Complexity (TAGC), UMR1090, 13288 Marseille, France

Corresponding authors:

fabienne.malagnac@universite-paris-saclay.fr

Key words: Histone methylation, sexual development, ChIP-seq, Podospora anserina 


\section{Abstract}

Selective gene silencing is key to development. The H3K27me3 enriched heterochromatin maintains transcription repression established during early development and regulates cell fate. Conversely, H3K9me3 enriched heterochromatin prevents differentiation but constitutes a permanent protection against transposable element. We exploited the fungus Podospora anserina, a valuable alternative to higher eukaryote models to question the biological relevance and interplay of these two distinct heterochromatin conformations. We found that H3K27me3 and H3K9me3 modifications are mutually exclusive within gene-rich regions but not within repeats. Lack of PaKmt6 EZH2-like enzyme resulted in loss of H3K27me3 and in significant H3K9me3 reduction, whereas lack of PaKmt1 SU(VAR)3-9-like enzyme caused loss of $\mathrm{H} 3 \mathrm{~K} 9 \mathrm{me} 3$ only. We established that $P$. anserina developmental programs require H3K27me3 mediated silencing unlike most fungi studied to date. Our findings provide new insight into roles of these histone marks and into the relationship between chromatin modifications and development. 


\section{Introduction}

Histones are subjected to a variety of post-translational covalent modifications (Strahl and Allis 2000) that impact the overall degree of packing of the genome. In most organisms, the opened euchromatin is enriched in methylation of H3K4 (Lysine 4 of histone 3) nearby the transcriptional start sites (TSS) of the active genes and is associated with methylated H3K36 located in the body of the transcribed genes (Kouzarides 2007). By contrast, histones present in the compacted heterochromatin are enriched in either tri-methylated H3K27 (H3K27me3) or tri-methylated H3K9 (H3K9me3) (Bhaumik, Smith, and Shilatifard 2007). The compact architecture of heterochromatin limits the accessibility of the transcription machinery to the embedded DNA, thereby silencing gene expression. Repeat-rich genomic regions enriched in H3K9me3 are referred to as 'constitutive' heterochromatin because the subsequent silencing is constant across development (Saksouk, Simboeck, and Déjardin 2015). In contrast, the 'facultative' heterochromatin corresponds to the deposition of H3K27me3 on gene-rich regions, whose silencing is transient and dynamic across developmental processes, allowing cell type-specific differentiation and rapid adaptation of gene expression (Trojer and Reinberg 2007)

H3K9me3 is catalyzed by the SET-domain SU(VAR)3-9 enzymes (Martens et al. 2005; Nakayama et al. 2001; Rea et al. 2000; Rice et al. 2003) and bound by the chromodomain of the Heterochromatin Protein 1 (HP1, (James and Elgin 1986; Eissenberg et al. 1990; Singh et al. 1991). Subsequent HP1 oligomerization, which results in nucleosome binding (Canzio et al. 2011) and phase separation (Larson et al. 2017; Sanulli et al. 2019; Strom et al. 2017) further enhances chromatin compaction and spreads this structure over large genomic compartments. When present, high level of DNA cytosine methylation is found in $\mathrm{H} 3 \mathrm{~K} 9 \mathrm{me} 3$ enriched regions. This modification is essential to genome stability by preventing either expression of transposable elements or illicit mitotic recombination. As demonstrated in mouse and drosophila models, when H3K9me3 enzymatic activity is absent or reduced, the embryos died because of various developmental 
defects (Liu et al. 2014) Moreover, since H3K9me3-dependant heterochromatin prevents the DNA binding of a large palette of transcription factors, it has long been considered as a barrier to cell fate changes (Becker, Nicetto, and Zaret 2016).

First identified in Drosophila, the conserved Polycomb group (PcG) protein complexes were shown to be both writers (Polycomb Repressive Complex 2, PRC2) and readers (Polycomb Repressive Complex 1, PRC1) of H3K27me3 (Lewis 1978; Ringrose and Paro 2004; Schuettengruber and Cavalli 2009). The catalytic subunit of the PRC2 is made of the SET domain protein enhancer of zest homolog 2 (EZH2), which tri-methylates H3K27. The H3K27me3-polycomb modification is instrumental for maintaining transcription repression established during early development and therefore has been linked to biological function related to cell fate, as different as X-inactivation (Wang et al. 2001) and hematopoiesis (Majewski et al. 2008). Conversely, when PCR2 functions are impaired, development of plants (Goodrich et al. 1997), insects (Birve et al. 2001) and mammals (O'Carroll et al. 2001a; Pasini et al. 2004) is disrupted, presumably because lineage specific programs are no longer accurate. Moreover, mutations affecting EZH2 leads to cell proliferation in cancer

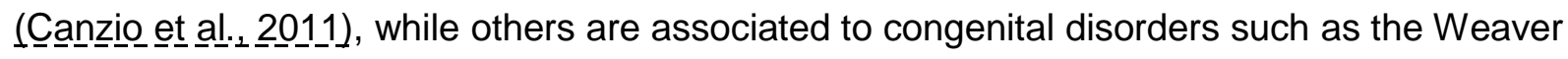
syndrome (Gibson et al. 2012) or the Ataxia-telangiectasia disease (Li et al. 2013).

In animals and flowering plants, H3K9me3 and H3K27me3 marks are quite mutually exclusive (Wiles and Selker 2017) and although these two histone modifications both result in gene expression silencing it is likely through different routes. Given the complexity and the essential nature of either of these pathways in metazoans, their respective mechanisms remain poorly understood. Even yeasts, although being otherwise powerful model systems, have some limitation in terms of chromatin remodeling studies. Indeed, if Schizosaccharomyces pombe possesses the Clr4 enzyme that catalyzes H3K9me3 (Rea et al. 2000), no such modification exists in Saccharomyces cerevisiae. As for the PRC2 complex, it is absent in all ascomycete yeasts. By contrast, filamentous fungi of the Pezizomycotina clade present canonic features for both H3K9me3- and H3K27me3dependent heterochromatin (Shaver et al., 2010). Unlike yeasts, filamentous fungi 
differentiate complex multicellular structures that require mobilization of specific lineage programs. In addition, the possibility to set up very efficient and straightforward genetic approaches (i.e. construction of mutants bearing multiple deletions) was already proven to be instrumental to address complex questions such as functional redundancy and pathway interplay. These characteristics make filamentous fungi a valuable experimental alternative to higher eukaryote models.

Genetic screens performed in Neurospora crassa, established that H3K9me3 methyltransferase DIM-5, and HP1-homolog are both essential for developmental programs that ensure normal growth, full fertility and DNA methylation (Tamaru and Selker, 2001, Freitag et al., 2004). By contrast, in Aspergillus nidulans, deletions of genes encoding either the DIM-5-homolog ClrD and the HP1-homolog HepA result in enhanced expression of a subset of secondary metabolite (SM) clusters but not in developmental defect (ReyesDominguez et al. 2010). However in the closely related maize pest Fusarium verticillioides, FvDIM5 deletion mutants display defects in both developmental programs (i.e. growth, conidiation, perithecium production) and overproduction of some SM clusters (Gu et al. 2017). The role of H3K27me3-dependant heterochromatin is also contrasted. In N. crassa, disruption of H3K27me3 methyltransferase activity did not lead to developmental defects (Jamieson et al. 2013) but to abnormal chromosome conformation (Klocko et al. 2016). By contrast, in the pathogenic fungus Zymoseptoria tritici absence of H3K27me3 reduces the loss of its accessory chromosomes, whereas loss of H3K9me3 induced many genomic rearrangements (Möller et al., 2019). Moreover, in N. crassa and Z. tritici, depletion of $\mathrm{H} 3 \mathrm{~K} 9 \mathrm{me} 3$ resulted in massive redistribution of $\mathrm{H} 3 \mathrm{~K} 27 \mathrm{me} 3$ in genomic compartments that are embedded in constitutive $\mathrm{H} 3 \mathrm{~K} 9 \mathrm{me} 3$ heterochromatin in their respective wild-type backgrounds (Jamieson et al., 2016, Möller et al., 2019). In contrast, the absence of H3K27me3 machinery did not impair the H3K9me3 genomic localization in either of them. H3K27me3 loss in the plant pathogens Fusarium graminearum and Fusarium fujikoroi resulted in growth defects, sterility and in constitutive expression of genes encoding mycotoxins, pigments-producing enzymes and other SM (Connolly et al., 2013, Studt et al., 
2016b). Hence, although described as being distributed in distinct genomic compartments in fungal wild-type backgrounds, both $\mathrm{H} 3 \mathrm{~K} 9 \mathrm{me} 3$ and $\mathrm{H} 3 \mathrm{~K} 27 \mathrm{me} 3$ can cooperate to regulate complex traits such as symbiosis (Chujo and Scott 2014a).

To further explore the functions and interplay of the constitutive versus facultative heterochromatin, we used the model system $P$. anserina to establish the genome-wide distribution of H3K4me3, H3K9me3 and H3K27me3. Surprisingly, most of the H3K9me3 are found in regions also enriched in $\mathrm{H} 3 \mathrm{~K} 27 \mathrm{me} 3$, while both the $\mathrm{H} 3 \mathrm{~K} 9 \mathrm{me} 3$ and $\mathrm{H} 3 \mathrm{~K} 27 \mathrm{me} 3$ are co-exclusive with the H3K4me3. We demonstrated that absence of PaKmt1, the H3K9me3 methyltransferase, resulted in loss of $\mathrm{H} 3 \mathrm{~K} 9 \mathrm{me} 3$, which had a limited impact on $P$. anserina growth, differentiation and sexual reproduction. Surprisingly we observed that absence of PaKmt6, the H3K27me3 methyltransferase, resulted in both H3K27me3 and H3K9me3 drastic depletion and caused severe defects in most of the aspects of its life cycle including growth, differentiation processes and sexual reproduction. 


\section{Results}

Heterochromatin remodelers are present in $P$. anserina genome and expressed during its life cycle

$P$. anserina genome contains a single gene ( $\left.P a \_6 \_990\right)$ encoding a putative H3K9me3 methyltransferase, named PaKmt1 (342 aa) and a single gene (Pa_1_6940) encoding a putative H3K27me3 methyltransferase, named PaKmt6 (1090 aa). Both of these highly conserved proteins show a canonical SET domain typical of the bona fide histone methyltransferases (Fig. 1A, Fig S1A, B and C). PaKmt1 displays a pre and post-SET domains (PS50867 and PS50868). PaKmt6 harbors a CXC domain (PS51633), which consists of two copies of a cysteine-rich motif frequently found in polycomb group (Pc-G) proteins.

RNA-seq experiments detected PaKmt6 transcripts of predicted structure in both vegetative mycelium and fruiting bodies (P. Silar et al. 2019). However, while annotation of the PaKmt1 gene predicted that its ORF was made of two exons, RNA-seq experiments showed ambiguous profiles for PaKmt1 transcripts (P. Silar et al. 2019). Dedicated reversetranscription polymerase chain reaction (RT-PCR) experiments revealed two distinct transcripts (Fig. S2). From one-day-old mycelium RNA extract, we amplified a single 1108-bp fragment corresponding to the PaKmt1 spliced transcript. However, from either four-day-old mycelium RNA extract or fruiting bodies RNA extracts (two days and four days postfertilization fruiting bodies), we amplified an additional 1170-bp fragment, which corresponds to a PaKmt1 unspliced transcript. If translated, the 1170-bp fragment would result in a truncated protein of 76 amino acids lacking the conserved functional motifs (pre-SET and SET domains). This observation suggests that, in addition to the functional full length PaKmt1 H3K9 methyltransferase, $P$. anserina may produce a truncated version of this protein at specific developmental stages of its life cycle.

Genome-wide distribution of H3K4me3, H3K9me3 and H3K27me3 histone modifications in P. anserina genome 
By using western-blotting, we first showed that H3K4me3, H3K9me3 and H3K27me3 marks were readily present in $P$. anserina genome (Fig. 1B, see WT line). Then, to further characterize the genomic patterns of these three histone modifications, we performed ChIPseq experiments on chromatin samples collected from vegetative mycelia grown $60 \mathrm{~h}$ at $27^{\circ} \mathrm{C}$ (Fig. 2A \& 2B, Fig. S3, Table S1 \& S2).

The H3K4me3 modification covered nearly $11 \%$ of the $P$. anserina genome (Fig. S4). With 3050 detected peaks (Table S1), this mark appeared to be present in blocks of approximately $1.3 \mathrm{~kb}$ in gene-rich regions (Fig. 2A, Fig. 3A), mostly located in 5' of CDS (Fig. 3B). As in other eukaryotes, H3K4me3 showed a positive correlation with active transcriptional activity (Fig. $3 \mathrm{C}$ ). On rare instances, when H3K4me3 peaks appeared to be localized in repeats, it mainly corresponded to transposable elements (TE) relics or transposase genes inserted into CDSs (49 out of the $63 \mathrm{H} 3 \mathrm{~K} 4 \mathrm{me} 3$ peaks found in repeats).

Conversely, H3K9me3 (Fig. 2A \& 2B, Fig. S3) was the less abundant mark found in P. anserina genome (1090 detected peaks, $2.94 \%$ of the genome (Table S1 and Fig. S4)) where it formed approximately 0.9 kb-long blocks (Fig. 3A), mainly found at repeat-rich regions, i.e. TE (728 peaks), centromeric regions and telomeric regions (Fig. 2A, Fig. 3B). Although low amount of H3K9me3 could be observed in some CDS (Fig. 3B), MACS2 peak calling analysis did not detect any significant enrichment (Table S1). In accordance with its genomic distribution, the H3K9me3 modifications showed a negative correlation with active transcriptional activity (Fig. 3C).

Being the second most common mark found in the $P$. anserina genome in terms of peak numbers (1941 detected peaks, Table S1), H3K27me3 (Fig. 2A \& 2B, Fig. S3) covered none of the less the most sizeable part of it (18\%, Fig. S4), showing large blocks of approximately $3.5 \mathrm{~kb}$-long (Fig. 3A). This mark was found enriched at sub-telomeric regions and on a fraction of TE clusters (Fig. 2B \& 3B), but also on about one third of the annotated CDSs (2992 detected peaks, Table S2). As such, most of these H3K27me3-marked CDS were likely not expressed during the vegetative growth phase (Fig. 3C). 
On a broad scale, we noticed that H3K4me3, H3K9me3 and H3K27me3 modifications were not equally distributed on $P$. anserina chromosomes as shown in Fig. S4. Intriguingly, H3K27 methylation appeared enriched on chromosomes four and six and even more so on chromosome five. However, by contrast to Zymoseptoria tritici (Schotanus et al. 2015), the $P$. anserina's chromosomes enriched in H3K27me3 are not dispensable. The same trend was observed for $\mathrm{H} 3 \mathrm{~K} 9 \mathrm{me} 3$ on chromosome five, which is indeed enriched in TEs and repeats. Conversely, H3K4me3 appeared less abundant on chromosome five.

Zooming in $P$. anserina's genomic patterns, it appeared that distribution of H3K4me3 was found mutually exclusive with both $\mathrm{H} 3 \mathrm{~K} 9 \mathrm{me} 3$ and H3K27me3 (Fig. 2B see close up, Fig. 3B \& 3D and Fig. S3). However, we did find genes that harbored both H3K4me3 and H3H27me3 marks in these experimental conditions (Table S2). Remarkably, this set was significantly enriched ( $p$-value $<0.001$ ) in genes encoding proteins involved in non-allelic heterokaryon incompatibility $(\mathrm{HI})$, transcription and chromatin remodeling (Table S2). Notably, patterns of H3K27me3 and H3K9me3 modifications could be found overlapping in gene-poor compartments scattered across the genome (Fig. 2A and Fig. S3), especially on repeats, which included transposase promoters and CDS (Fig. 3B: cluster_1, and Table S2). Indeed, we identified $954 \mathrm{H} 3 \mathrm{~K} 27 \mathrm{me} 3$ and H3K9me3 common peaks, which represented 90\% of the H3K9me3 peaks (Table S1).

\section{PaKmt1 and PaKmt6 are histone methyltransferases and their deletion impact both H3K9me3 and H3K27me3 distribution.}

To determine the impact of the lack of heterochromatin marks, we constructed the $\Delta$ PaKmt1 and the $\Delta$ PaKmt6 deletion mutants (Fig. S5). Double $\Delta$ PaKmt1 $\Delta$ PaKmt6 mutant strains were obtained by crossing the two corresponding single mutants. All three mutants were alive, showing that neither PaKmt1 nor PaKmt6 are essential genes. Western-blotting showed that $\mathrm{H} 3 \mathrm{~K} 9 \mathrm{me} 3$ was almost absent in proteins extracted from the single $\triangle P a K m t 1$ mutant (Fig. 1B). Likewise, the H3K27me3 was almost absent in extracts from the single $\Delta$ PaKmt6 mutant. Neither mark was present in the double $\Delta$ PaKmt1 $\Delta$ PaKmt6 mutants. As expected, H3K4me3 was detected in all samples. These observations clearly show that 
PaKmt1 displays a H3K9me3 methyltransferase enzymatic activity, while PaKmt6 is displays a H3K27me3 methyltransferase enzymatic activity.

ChIP-seq experiments performed in the $\triangle P a K m t 1$ background showed low amount of H3K9me3 (Fig. 4A and 4C, Fig. S3 and Fig S4), however MACS2 peak calling analysis did not detected any significant peak enrichment (Fig 4A, Table S1 and Fig. S4), by contrast to the same analysis performed in the wild-type background. This would mean that PaKmt1 is responsible for the wild-type distribution of $\mathrm{H} 3 \mathrm{~K} 9 \mathrm{me} 3$ modifications at the specific locations described above. Together with the western-blot analysis, these results would further suggest that PaKmt1 is the main P. anserina's H3K9me3 methyltransferase. Unexpectedly, our data revealed variations of the $\mathrm{H} 3 \mathrm{~K} 27 \mathrm{me} 3$ patterns in the $\Delta P a K m t 1$ mutant background (Fig. 4A, 4B and Fig. S4). Firstly, the average length of the H3K27me3 blocks was reduced by two third (Fig. 4C). Secondly, about $30 \%$ of the H3K27me3 peaks were missing, especially those located in the gene-rich blocks (Fig. 4A, 4B, Table S1 and Fig. S3), although chromosomes four, five and six still were enriched in H3K27me3 compared to the others (Fig. S4). Thirdly, the remaining H3K27me3 peaks did not show any extensive re-localization when compared with the H3K27me3 wild-type genome-wide distribution (Fig 4A, Fig. 4B CDS and Fig. S3). However, some H3K27me3 enrichment was observed in one cluster of repeated elements (Fig. 4B cluster_3). This observation is in stern contrast to the extensive re-localization of the H3K27me3 marks that was reported as a consequence of H3K9me3 loss in both N. crassa and Z. tritici (Jamieson et al. 2013; 2016; Möller et al. 2019).

ChIP-seq experiments performed in the $\Delta$ PaKmt6 mutant strains revealed that this deletion drastically reduced the overall H3K27me3 (Fig. 4A, 4B \& 4C and Fig. S3) content (significant genome coverage dropped from $18.95 \%$ to $0.04 \%$, Table S1 and Fig. S4). The presence of these residual H3K27me3 marks was reminiscent of what we observed by western-blot analysis, where a faint band was still visible in the $\Delta P a K m t 6$ mutants but disappeared in the $\triangle P a K m t 1 \triangle P a K m t 6$ double mutants (Fig. 1B). We performed a dot-blot analysis that ruled out a potential cross-hybridization, because the H3K27me3 antibody used in this study cannot detect the H3K9me3 epitopes (Fig. S6). Altogether, these findings 
suggest that PaKmt1 may have a residual H3K27me3 methyltransferase activity. But intriguingly, most of the remaining $\mathrm{H} 3 \mathrm{~K} 27 \mathrm{me} 3$ marks were no longer localized into the H3K27me3 blocks detected in the wild-type genetic background (Fig. 4A and Fig. S3) and even found enriched in one cluster of repeated elements (Fig. 4B cluster 3). To our surprise, we also noticed that the loss of PaKmt6 severely impacted the amount of H3K9me3 marks, since in the $\triangle P a K m t 6$ background, its genome coverage dropped from $2.94 \%$ to $0.18 \%$ (Fig. 4A, Table S1 and Fig. S4). This means that the diminution of the H3K9me3 peaks was as strong as that of the $\mathrm{H} 3 \mathrm{~K} 27 \mathrm{me} 3$ in this mutant background. Nevertheless, the remaining H3K9me3 peaks in the $\triangle P a K m t 6$ background were not displaced, nor the missing H3K9me3 blocks replaced by H3K27me3 ones (Fig. 4A).

Finally, H3K4me3 patterns stayed unchanged in all the mutant backgrounds, suggesting that loss of either H3K27me3 or H3K9me3 has no impact on the H3K4 methyltransferase activity (Fig. 4A \& 4C, Table S1 and Fig. S4) and global chromatin relaxation did not interfere with genome-wide H3K4me3 patterns.

\section{Loss of H3K27me3 in $\triangle P a K m t 6$ background modifies gene expression}

To assay the impact of $\mathrm{H} 3 \mathrm{~K} 27 \mathrm{me} 3$ or/and H3K9me3 loss on gene expression, we performed qRT-PCR experiments on vegetative mycelia grown 60 hours at $27^{\circ} \mathrm{C}$, of either wild-type genotype (5 independent samples) or mutant genotypes ( $\triangle P a K m t 1$ and $\triangle P a K m t 6$, 5 independent samples each). We first focused on six genes that harbor H3K27me3 marks in wild-type background (Fig. 5A, Fig S7and Table S2). In the $\triangle$ PaKmt6 background (Fig. 5A, Table S2), all of them lost their H3K27me3 marks and were over expressed (fold change $\geq 2$ ). This result is consistent with $\mathrm{H} 3 \mathrm{~K} 27 \mathrm{me} 3$ being involved in regulation of gene expression during development programs since $\mathrm{Pa}$ _1_6263, which showed the most dramatic up-regulation effect (fold change $>25$, p_value $=0.001 \mathrm{Fig} .5 \mathrm{~A}$ ), is supposed to be expressed during the sexual phase only (P. Silar et al. 2019). Expression of genes encoding secondary metabolites (SM) is known to be sensitive to chromatin compaction (Gacek and Strauss 2012; Connolly, Smith, and Freitag 2013; Studt, Janevska, et al. 2016; Studt, Rösler, 
et al. 2016). Indeed, when we assayed the expression of two such genes ( $P a \_6 \_7270$ and Pa_6_7370) in the $\triangle$ PaKmt6 background, we found them both up regulated (Fig. 5A, Table S2). Notably, loss of PaKmt1 had no significant impact (fold change $\leq 2$ ) on the expression of this set of genes, even if some of the H3K27me3 marks were lost in this mutant background (Fig. S7 and Table S2).

\section{Loss of PaKmt6 has stronger impact on vegetative growth than loss of PaKmt1}

We then assessed the impact of loss of either H3K27me3 or H3K9me3, or both, on $P$. anserina's life cycle. Pigmentation, branching and the overall aspect of $\Delta P a K m t 1$ mycelia were similar to those of the wild-type strains, except for reduced production of aerial hyphae (Fig. 5B). The $\triangle$ PaKmt1 mutants also displayed a slight growth defect at optimal temperature $\left(27^{\circ} \mathrm{C}\right)(6 \%$ decrease for $\triangle \mathrm{PaKmt1}$, Fig. $5 \mathrm{C})$. This growth defect was stronger when the $\triangle$ PaKmt1 mutants were grown at cold temperature $\left(11^{\circ} \mathrm{C}, 28 \%\right.$ decrease, Table 1$)$. Upon cultivation on solid media, we noticed that the $\triangle P a K m t 1$ mutants showed a delay in resuming vegetative development after a period of growth arrest (cells in stationary phase or storage at $4^{\circ} \mathrm{C}$ ). To further explore this phenotype, we set up a stop and re-start growth assay described in (Fig. S8A). The wild-type strain was able to resume proper mycelium growth either from implants cut out of the apex of the colony (Plug\#1, Fig. S8A) or out of two distinct locations of the inner stationary phase (Plug\#2 \& Plug\#3, Fig. S8A). By contrast, $\triangle$ PaKmt1 mycelia developing from implants cut out of the inner stationary phase of the colony (Plug\#3, Fig. S8A) were growing slow and generated irregular colony margins and almost no aerial hyphae. This already defective and erratic growth was further degraded after five days of culture. However, mycelia of $\triangle P a K m t 1$ mutants that developed from implants cut out of the apex of the colony (Plug\#1, Fig. S8A) grew ordinarily. When plugs of any of these thalli where cut out of the growing edge, they all regenerated normally (Fig. S8A), confirming that this defect was not permanent but rather linked to the disability of the $\Delta P a K m t 1$ strains to resume growth properly. Because these observations were reminiscent of those of Crippled Growth (CG), an epigenetically triggered cell degeneration (Nguyen et al. 2017), we made sure that the $\triangle P a K m t 1$ mutation did not promote $C G$ (Fig. S8B) but behaved as the wild-type 
strain in that respect. Therefore, this reduced capability to resume growth may be indicative of a lesser plasticity to perform adaptive developmental program under changing environmental conditions.

Pinkish pigmentation (see the following paragraph), crooked branching pattern and the overall aspect of $\Delta P a K m t 6$ mycelia were quite different to those of the wild-type strains (Fig. 5B). First of all, $\triangle$ PaKmt6 mutant growth-rate was $43 \%$ lower than wild-type strains during the first 60 hours of growth on $\mathrm{M} 2$ at $27^{\circ} \mathrm{C}$ (Fig. 5C. Remarkably, after this time point, the mycelium development became so erratic and irregular that the standard assay consisting in thallus radius measures seemed no more accurate. Indeed, $\Delta$ PaKmt6 mycelia showed "stop-restart" growth resulting in patchy mycelium sectors, which literally sprouted out of the colony margins like cauliflower inflorescences (Fig. 5B). Large error bars of the corresponding growth-curve represented these outgrowths (Fig. 5C). Surprisingly, we were able to grow the $\triangle P a K m t 6$ mutants in race tubes to determine that this mutation had no effect on senescence (Fig. S9). Finally, when we constructed the $\Delta$ PaKmt1 $\Delta$ PaKmt6 double mutant, the phenotypes generated by the $\Delta$ PaKmt6 null allele were epistatic upon those generated by the $\triangle P a K m t 1$ allele.

\section{The $\Delta$ PaKmt6 mutant produces more male gametes than the wild-type strain}

In addition to the growth defect, the $\triangle P a K m t 6$ mutant mycelium showed a powdery aspect that was different from that of the wild-type strain. When examined under the microscope, we observed a striking over-production of male gametes (spermatia) that accounted for the powdery phenotype of the $\Delta$ PaKmt6 mutants. In this mutant context, male gametes production was about 130 times higher than in wild-type background (Fig. 6A). We then tested if these mutant male gametes were as fertile as the wild-type ones. To do so, we fertilized wild-type female gametes (ascogonia) with a defined quantity of either mutant or wild-type male gametes of compatible mating type. After seven days of growth at $27^{\circ} \mathrm{C}$, fruiting bodies were numbered, given that each of them originated from a single fertilization event. Fertilization capabilities of the mutant male gametes were as efficient as the wild-type ones, since similar numbers of fruiting bodies were obtained in both conditions (4 
independent experiments). This indicates that the $\Delta P a K m t 6$ mutant male gamete over production had no impact on their biological properties.

The $\Delta$ PaKmt6 mutant formed crippled fruiting bodies yielding a reduced number of ascospores

We then investigated the ability of the histone methyltransferase mutant strains to perform sexual reproduction. Sexual development and ascospore production of crosses involving two $\triangle$ PaKmt1 parental strains were indistinguishable from those of wild-type strains. By contrast, when we performed homozygous $\triangle P a K m t 6$ crosses, fruiting bodies were formed, which means that fertilization occurred, but their developmental time frame was delayed. They also displayed a large palette of morphological defects e.g., lack of necks or extra necks, lack of ostioles, upward/downward mis-orientation, development into agar, fusion, etc. (Fig. 6B) and their ascospore production was reduced. Heterozygous orientated crosses showed that when the $\Delta P a K m t 6$ null allele was present in the female gamete genome and the wild-type allele was present in the male gamete genome, the fruiting bodies were crippled as in the homozygous $\triangle P a K m t 6$ crosses, which resulted in a reduced ascus production (Fig. S10A). In contrast, a heterozygous cross involving a female wild-type strain produced fully fertile fruiting bodies, indicating a $\Delta$ PaKmt6 maternal effect.

Crosses involving the double $\Delta P a K m t 1 \Delta P a K m t 6$ mutant strains displayed a typical $\triangle$ PaKmt6 phenotype with respect to the perithecium number, morphology and ascospore production, demonstrating that $\triangle P a K m+6$ null allele was epistatic. In addition, one third of the asci recovered from homozygous $\triangle P a K m t 1 \triangle P a K m t 6$ crosses showed less pigmented ascospores than those of wild-type crosses (with a color panel spanning from white to green). In general, deficit in pigmentation is indicative of incomplete maturation during the ascospore formation process.

\section{PaKmt6 is essential to the development of the envelope of fruiting bodies}

To check whether the $\triangle P a K m t 6$ mutant's reduced fertility resulted from either a fruiting body envelope defect or a zygotic tissue defect, we set up tricaryon crosses involving the $\Delta$ mat strain (E. Coppin et al. 1993; Jamet-Vierny et al. 2007). Because the $\Delta$ mat strain 
lacks the genes required for fertilization, it do not participate either as male or female in the sexual reproduction. However, the $\Delta$ mat mycelium is able to provide maternal hyphae required to build fruiting bodies. Consequently, the $\Delta$ mat strain can complement mutants defective for fruiting body tissues formation but not for zygotic tissue defect. We observed that the $\Delta$ mat ; $\triangle$ PaKmt6 mat+ ; $\triangle P a K m t 6$ mat- tricaryon yielded few but fully matured and well-formed fruiting bodies only that produced as many asci as the wild-type ones (Fig. S10B). This experiment demonstrates that $\triangle P a K m t 6$ mutation compromised the fruiting body envelope formation but had no impact on zygotic development.

\section{The PaKmt6 null allele reduces the ascospore germination efficiency}

Haploid ascospores originating from homozygous $\Delta P a K m t 1$ crosses germinated as those of wild-type crosses (> 96\% germination rate, $\mathrm{N}=100$, Table 2). However, about two third of the wild-type looking ascospores produced by the homozygous $\Delta$ PaKmt6 crosses did not germinate ( 3 independent experiments, $\mathrm{N}=100$ for each experiment, Table 2 ). The reduced germination efficiency was further enhanced in $\Delta$ PaKmt1 $\triangle$ PaKmt6 double mutant (Table 2). On germination medium, wild-type ascospores germination occurs at a germ pore located at the anterior side of the ascospore, by the extrusion of a spherical structure. This transient structure, called a germination peg, gives rise to a polarized hypha that develops into mycelium. In the $\triangle P a K m t 6$ mutants, the ascospore germination process was stopped at an early stage, since germination pegs were not formed. However, the $\Delta$ PaKmt6 defect was ascospore autonomous since ascospores from wild-type progeny obtained in WT $\mathrm{x} \Delta$ PaKmt6 crosses germinated normally, whereas those from $\Delta$ PaKmt6 progeny did not. However, $\mathrm{PaKmt6}^{+} / \triangle \mathrm{PaKmt6}$ dikaryotic ascospores had a wild-type germination rate showing that the $\Delta$ PaKmt6 defect was recessive (2 independent experiments, $\mathrm{N}=80$ dikaryotic ascospores). It has been previously shown that unpigmented ascospores carrying the pks1-193 mutant allele germinate at high frequency (over 95\%) on both inducing and non-inducing media (Malagnac et al. 2004). Indeed, the $P$. anserina pks1-193 mutants carry a recessive null mutation in the gene encoding the polyketide synthase involved in the first step of DHN melanin biosynthesis (Evelyne Coppin and Silar 2007). Therefore, these mutants form 
unmelanized ascospores that germinate spontaneously. When crosses between pks1-193 $\triangle$ PaKmt6 mat- and $p k s 1^{+} \Delta$ PaKmt6 mat+ strains were performed, they generated the expected asci made of two unpigmented and two pigmented ascospores. If the pigmented ascospores (pks $\left.1^{+} \Delta P a K m t 6\right)$ had to be transferred to inducing germination medium for some of them to germinate (app. 30\%), the unpigmented ones (pks1-193 $\Delta$ PaKmt6) germinated efficiently (over $90 \%$ ) even on non-inducing medium. This result shows that the absence of melanin suppressed the $\triangle P a K m t 6$ ascospore germination defect, as for those of the $\Delta P a P l s 1$ and the $\triangle$ PaNox2 mutants (Lambou et al. 2008).

\section{H3K9me3 reader HP1 is present in $P$. anserina}

In fungi, the PRC1 complex is not present (Wiles and Selker 2017). However, we searched the $P$. anserina genome for a homolog of the heterochromatin protein 1 (HP1, (Honda and Selker 2008)) which is known to bind the H3K9me3 marks of the constitutive heterochromatin. We identified one gene ( $\mathrm{Pa} \_$4_7200) encoding the protein PaHP1 (252 aa, $57 \%$ identity, e-value $\left.=4 e^{-78}\right)($ Fig. 1A). It displays conserved chromo domain (PS00598) and chromo shadow domain (PS50013) (Fig. S11A \& S11B). By self-aggregation, chromo shadow domain containing proteins can bring together nucleosomes and thus condense the corresponding chromatin region (Canzio et al. 2011). As for PaKmt6, RNA-seq data showed that the PaHP1 gene is expressed in both vegetative mycelium and fruiting bodies tissues $(P$. Silar et al. 2019).

\section{Loss of PaHP1 results in fewer but broader H3K9me3 domains}

To explore potential role(s) of PaHP1, we deleted the corresponding Pa_4_7200 gene (Fig. S12A \& 12B). No difference in H3K4me3, H3K9me3 or H3K27me3 was detected by western-blotting performed on nuclear proteins extracted from either the wild-type strains or the single $\triangle P a H P 1$ mutant strains (Fig. 1B). When we performed ChIP-seq experiment in the $\triangle P a H P 1$ background, the H3K27me3 pattern was similar to that of $\triangle P a K m t 1$ strains, while the H3K4me3 pattern was similar to that of the wild-type strains (Fig. 7A, 7B and Fig S3). However, about half of H3K9me3 genome coverage was lost (Fig. S3 and S4, Table S1), whereas some $\mathrm{H} 3 \mathrm{~K} 9 \mathrm{me} 3$ enrichment was observed in one cluster of repeated elements 
(Fig. 7B cluster_4, Table S1). Yet, if the density of H3K9me3 was reduced, the average size of the peaks was quite larger (Fig. 7C). This suggests that, in the absence of the recruitment platform mediated by the binding of PaHP1 to the constitutive heterochromatin, the compact structuration may be partly relaxed and the histone modifications lost, but also that the boundaries between heterochromatin and euchromatin may be less clear-cut. However, in this mutant context the $\mathrm{H} 3 \mathrm{~K} 9 \mathrm{me} 3$ reduction has no impact on gene expression (Fig. S13).

\section{Loss of PaHP1 has no more impact than that of PaKmt1}

Throughout all the $P$. anserina's life cycle, the $\triangle P a H P 1$ mutants behave similarly to the $\triangle$ PaKmt1 ones (Table 1, Fig. S12A, S12C, S12D, S12E). Importantly, the double $\triangle P a K m t 1 \triangle P a H P 1$ mutants did not show any aggravated phenotypes (Table 1, Fig. S13A). When the $\triangle P a H P 1$ allele was associated to the $\triangle P a K m t 6$ one, all the phenotypes displayed by the single $\triangle P a K m t 6$ mutants were epistatic upon those of the $\triangle P a H P 1$ mutants. The only exception is related to the germination efficiency of the triple $\triangle P a K m t 1 \Delta P a H P 1 \Delta P a K m t 6$, which was similar to the double $\triangle P a H P 1 \triangle P a K m t 6$ rather than the one of the double $\Delta$ PaKmt1 $\triangle$ PaKmt6 mutants (Table 2). This may be due to a slightly reduced pigmentation of the ascospores produced by the homozygous $\triangle P a K m t 1 \triangle P a H P 1 \triangle P a K m t 6$ crosses. Indeed, even if only the darker wild-type looking ones were used in this test, slightly reduced pigmentation facilitates germination.

\section{Subcellular localization of PaKmt1-mCherry and PaHP1-GFP}

Because enzymatic activity was fully restored in the $\triangle P a K m t 1$ strain by introduction of the PaKmt1-mCherry allele encoding the PaKmt1-mCherry chimeric protein (See Fig. 1B, Material and Method section and below), we explored its cellular localization in two-day-old growing mycelium, along with that of PaHP1-GFP. In both PaKmt1-mCherry and PaHP1GFP expressing strains, the fluorescence signal was nuclear and punctuated (Fig. 8). When merged, the two fluorescent signals co-localized. However, when the PaHP1-GFP allele was present in a $\triangle P a K m t 1$ background, the GFP signal was present but delocalized (Fig. 8) as already observed in N. crassa (Freitag et al. 2004). Instead of forming foci, the GFP fluorescence was still nuclear but fuzzy, suggesting that, without H3K9me3 modification, the 
PaHP1 protein is no longer recruited by the appropriate heterochromatic genomic territories. On the contrary, in the same experimental conditions (two-day-old mycelium), when present in a $\triangle$ PaKmt6, the PaHP1-GFP protein was localized as in a wild-type background. Therefore, PaKmt6, by itself or through H3K27me3 modification, is not involved in targeting the PaHP1 to its proper genomic compartments. 


\section{Discussion}

In eukaryotes, the deposition of histone modification is essential to chromatin features and regulation of gene expression that impacts cell fate. Because $P$. anserina unlike $N$. crassa displays no DNA methylation but carries conserved SET-domain SU(VAR)3-9 enzyme and PRC2 complex, we aimed at understanding the impact of heterochromatin assembly on $P$. anserina life cycle in relation with its genome organization.

\section{H3K27me3 and H3K9me3 modifications are not mutually exclusive}

To get the first hints of $P$. anserina's genome organization, we took advantage of ChIP-seq experiments to uncover genome-wide chromatin landscapes in a wild-type background. Our results highlighted two uncommon features of both the constitutive and the facultative heterochromatin. Firstly, in most animals and plants, the H3K9me3 modification covers fairly large domains, while the H3K27me3 modification forms shorter ones. But in $P$. anserina's genome we observed the opposite, since H3K27me3 blocks were in average three times longer than the H3K9me3 blocks. Secondly, if as expected the H3K9me3 modification was restricted to repeats-rich regions, mostly located near telomeres and centromeres (Freitag 2017), the H3K27me3 modification encompass both annotated CDS and H3K9me3 marked repeats. Co-occurrence of $\mathrm{H} 3 \mathrm{~K} 9 \mathrm{me} 3$ and $\mathrm{H} 3 \mathrm{~K} 27 \mathrm{me} 3$ at same genomic loci is of particular interest because these two heterochromatic marks were long considered mutually exclusive in flowering plants, mammals, and fungi (Simon and Kingston 2013; Wiles and Selker 2017). Yet recently this dogma has been challenged, since, as demonstrated by mass spectrometry experiments, H3K27me3 and H3K9me3 can coexist within the same histone octamer in embryonic stem cells (Voigt et al. 2012). Furthermore, in the ciliate Paramecium tetraurelia, H3K9me3 and H3K27me3 co-occur at TEs loci (Frapporti et al. 2019), as well as in the nematode Caenorhabditis elegans (Ho et al. 2014) and in the bryophyte Marchantia polymorpha (Montgomery et al. 2020). In mammals, these two marks can be found together, in association with DNA methylation, at a subset of developmentally regulated genes in mouse extra-embryonic early embryo lineages (Alder et al. 2010). 
Notably, some of the human cancer cells also display both of these repressive marks (Widschwendter et al. 2007; Ohm et al. 2007; Schlesinger et al. 2007). This co-occurrence can also be found in plants, as such H3K9me3 and H3K27me3 overlap, exclusively associated with DNA methylation, had also been described at $A$. thaliana's ETs loci (Deleris et al. 2012). The results of this study further reinforce the biological relevance of the dual H3K27/H3K9 methylation in specific heterochromatin loci and show that these superpositions of heterochromatin marks can also happen in a genomic context devoid of DNA methylation.

\section{Hypothetical bivalent-like genes in fungi?}

As expected, the H3K4me3 modification, typically associated with active chromatin, was found in gene-rich segments of $P$. anserina's genome. As described in most eukaryotes, this modification was shown to be mutually exclusive with both of the repressive marks H3K9me3 and H3K27me3. However in mammals, totipotent embryonic stem cells show bivalent genes marked by both active H3K4me3 and repressive H3K27me3 chromatin modification (Thalheim et al. 2017). Such bivalent genes are proposed to play a central role in developmental and lineage specification processes. Remarkably, fungal nuclei conserved their totipotency throughout their whole life cycle. Indeed, almost any hyphal fragment can give raise to complete and totipotent new individuals (Roper et al. 2011; Money 2002). The molecular basis of this peculiar feature is not yet understood. In this study, we did find genes harboring both $\mathrm{H} 3 \mathrm{~K} 4 \mathrm{me} 3$ and $\mathrm{H} 3 \mathrm{H} 27$ me3 marks and moreover a sizeable part of them belonging to the het gene family. In $P$. anserina, the HET domain has been shown to be essential in inducing the program cell death as a consequence of $\mathrm{HI}$ reaction (Paoletti, Saupe, and Clavé 2007). Being members of the conserved STAND proteins (Signaltransducing ATPases with Numerous Domains), they also display NACHT and WD40 repeat

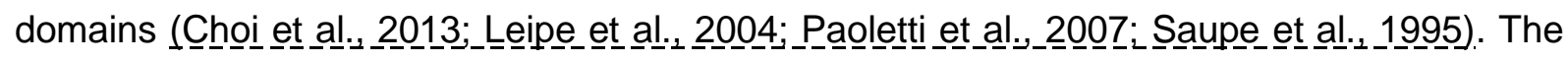
proteins of this family, which can oligomerize, are innate immune receptors, including NODlike receptors in animals and NB-LRR resistance proteins in plants (Rairdan and Moffett 2006). Likewise, in fungi, STAND proteins have been proposed to act as receptors to mediate the $\mathrm{HI}$ allorecognition process (Paoletti, Saupe, and Clavé 2007; Paoletti and Saupe 
2009). More generally, the $\mathrm{HI}$ may derive from a fungal innate immune system, where the STAND proteins could mediate non-self-recognition during interactions involving either individuals of the same species or of different species. By definition, the HI reaction must be quick and massive upon contacts. Therefore, expression of genes involved in this process could be finely tuned by marking them with both repressing and activating modifications.

\section{Chromatin status may confer distinct epigenetic identity to $\boldsymbol{P}$. anserina chromosomes}

On a broad scale, the patterns of histone modifications that we described in this study suggest that $P$. anserina chromosomes harbor distinct combinations of epigenomic features that may be related to functional compartmentalization of its genome.

The first combination, composed of high content of H3K4me3 and low content of both H3K27me3 and H3K9me3, is shared by chromosomes one, two, and seven. The second combination, made of reduced content of $\mathrm{H} 3 \mathrm{~K} 4 \mathrm{me} 3$ and enhanced content of both H3K27me3 and H3K9me3, is that of chromosomes four and six. The last combination, which corresponds to the highest contents in the repressive H3K27me3 and H3K9me3 marks and the lowest content in the active H3K4me3 mark concerns the chromosome five only. Remarkably, this chromosome contains a TE embedded spore-killer locus, responsible for drastic distortion of meiotic segregation (Grognet, Lalucque, et al. 2014a). Chromosome three showed composite features since it grouped with chromosomes one, two and seven regarding its $\mathrm{H} 3 \mathrm{~K} 4 \mathrm{me} 3$ and $\mathrm{H} 3 \mathrm{~K} 27 \mathrm{me} 3$ contents but with chromosomes four and six regarding H3K9me3 content. Drop of H3K4me3 on chromosome three was due to the presence of the rDNA array on this chromosome. This non-random distribution of repressive mark cannot only be accounted for by the TE/repeat content of these chromosomes. Indeed, if the fifth chromosome is enriched in repetitive sequences, the fourth and sixth are not. These observations are reminiscent of what had been described in Z. tritici (Schotanus et al. 2015). This fungus is endowed with 13 core chromosomes and up to eight accessory chromosomes, the latter showing significantly higher H3K9me3 and H3K27me3 enrichments, in line with the twofold higher proportion of repetitive DNA (Möller et al. 2019). In this context, it has been proposed that the epigenetic imprint of these accessory chromosomes would 
signal that they could be lost, in contrast to the core chromosomes that have to be retained ((Möller et al. 2019) and Table 3). No such dispensable chromosomes are present in the $P$. anserina genome.

\section{PaKmt1 and PaKmt6 are not independent actors of chromatin remodeling}

The ChIP-seq carried out here can allow us to test for potential crosstalk between constitutive and facultative heterochromatin. Elimination of PaKmt1 suppressed H3K9me3, which demonstrates that there is no other H3K9 methyltransferase activity in $P$. anserina. In the absence of the PaHP1, half of the H3K9me3 peaks identified in the wild-type background were missing, indicating that some positive feedback between the $\mathrm{H} 3 \mathrm{~K} 9 \mathrm{me} 3$ reader and its methylation enzyme may be needed to install or/and maintain the constitutive chromatin. Unexpectedly, our results also show that complete or partial loss of constitutive heterochromatin in $\triangle P a K m t 1$ mutants and in $\triangle P a H P 1$ mutants impacted facultative heterochromatin properties, since in these mutant backgrounds we identified fewer H3K27me3 peaks of reduced average size. Our data suggest that initiation and propagation of the PRC2 complex are inter-dependent processes in $P$. anserina. Alternatively, if, as shown in $A$. thaliana for the FLC locus (Angel et al. 2011), PRC2 complex initiation and propagation are independent in $P$. anserina, both of them are impaired when the H3K9me3heterochromatin pathway is damaged. However, $\mathrm{H} 3 \mathrm{~K} 27 \mathrm{me} 3$ reduction in both $\triangle P a K m t 1$ and $\triangle P a H P 1$ mutants was not associated with any substantial genomic redistribution by contrast to what has been described in the corresponding $N$. crassa and Z. tritici mutants (Jamieson et al. 2013; 2016; Möller et al. 2019) (Table3). This latter result means that although impaired in both $\triangle P a K m t 1$ and $\triangle P a H P 1$ mutants, the H3K27me3-heterochromatin is none of the less properly localized and does not spread over the vacant H3K9me3 genomic compartments.

Conversely, the PaKmt6 mutants showed massive loss of H3K27me3 but also some significant reduction of $\mathrm{H} 3 \mathrm{~K} 9 \mathrm{me} 3$, which could be explained if PaKmt6 targets PaKmt1 to its genomic compartment. Again theses data are in sharp contrast with those obtained in $N$. crassa (Jamieson et al. 2013; 2016) and Z. tritici (Möller et al. 2019) for which H3K9me3 content is not affected by loss of H3K27me3 (Table 3). Moreover, the few H3K27me3 marks 
that remained in the $\triangle P a K m t 6$ mutants were found delocalized when compared to their wildtype distribution. But importantly, these new locations did not overlap with the otherwise H3K9me3-marked genomic segments.

In general, two distinct enzymes catalyze methylation of either H3K9 or H3K27 residues. However, the ciliate $P$. tetraurelia is endowed with only one class of histone methlytransferase, the Enhancer-of-zeste-like protein Ezl1, which methylates both H3K9 and H3K27 residues (Frapporti et al. 2019). This observation suggests that during the course of evolution H3K9 and H3K27 can be substrates of the same methyltransferase. In the light of the above assertion, we can hypothesize that (i) the $P$. anserina's SU(VAR)3-like homolog (PaKmt1) may have a residual H3K27 histone-methyltransferase activity, that would account for the remaining delocalized $\mathrm{H} 3 \mathrm{~K} 27 \mathrm{me} 3$ marks in the $\triangle P a K m t 6$ mutants, (ii) SET-domain Enhancer-of-zeste-like PaKmt6 cannot be a catalytic substitute to PaKmt1, since H3K9me3 peaks were no longer detected in the $\Delta P a K m t 1$ mutants.

\section{Constitutive chromatin is dispensable whereas facultative chromatin is required for} most of the $P$. anserina developmental programs

Assessing the phenotype of $\triangle P a K m t 1, \triangle P a H P 1$, and $\triangle P a K m t 6$ single mutants, we established that the loss of both PaKmt1 and PaHP1 has almost no effects on P. anserina life cycle, whereas the absence of PaKmt6 drastically impairs every developmental step. Hence, if briefly recapitulated, the $\triangle P a K m t 6$ inactivation resulted in (i) slow, irregular, and thermo-sensitive mycelium growth, (ii) absence of aerial hyphae, (iii) striking over-production of male gametes, (iv) reduced fertility of the female gametes and/or of fertilization efficiency, (v) formation of crippled and misorientated fruiting bodies, and (vi) reduced yields of ascospores (vii) reduced germination efficiency of ascospores (Table 3). This means that almost every aspects of $P$. anserina life cycle are impacted by the loss of most of H3K27me3 marks and consequently of the facultative heterochromatin (see below). By contrast, the single $\triangle P a K m t 1$ and $\triangle P a H P 1$ mutants showed only marginal defects on aerial hyphae production and growth resuming after a period of arrest (Table 3). More importantly, the single $\triangle P a K m t 1$ and $\triangle P a H P 1$ and double $\triangle P a K m t 1 \triangle P a H P 1$ mutants are fully fertile. These 
phenotypes are the complete opposite of what had been described for those of $N$. crassa, as well as most filamentous fungi studied to date, i.e. Aspergillus fumigatus, Epichloë festucae, Leptoshaeria maculans, Penicillium oxalicum, Z. tritici (Table 3). Conversely, for F. graminearum and F. fujikuroi, Enhancer-of-zeste-like enzymes are key for their developmental integrity, as in P. anserina (Table 3). F. graminearum kmt6 mutants show aberrant germination patterns, over-pigmented mycelium, restricted growth, reduced pathogenicity, over-production of several SM clusters, and sterility, while loss of Kmt1 or HP1 orthologues does not lead to any noticeable phenotypes (Connolly, Smith, and Freitag 2013). Similarly, F. fujikuroi kmt6 mutants form stunted mycelia that produce a reduced yield of conidia (Studt, Rösler, et al. 2016).

\section{Conserved function of PRC2 complex in fungal development}

This intriguing contrasted picture suggests that two categories of fungi may be distinguished with respect to facultative heterochromatin functions (Table 3 ). In $P$. anserina, F. graminearum, and F. fujikuroi, methylation of $\mathrm{H} 3 \mathrm{~K} 27$ by the PRC2 complex represses developmentally regulated genes, especially by establishing and maintaining long-term gene silencing memory accordingly to cell fate specification. This is in line with what has been reported for flowering plants (Goodrich et al. 1997), insects (Birve et al. 2001), and higher eukaryotes (O'Carroll et al. 2001b) where disruption of PRC2 activity leads to abnormal differentiation of embryos and abnormal cell proliferation in cancer (Conway, Healy, and Bracken 2015). In other fungi, as in N. crassa (Basenko et al. 2015), E. festucae (Chujo and Scott 2014b), Z. tritici (Möller et al. 2019) and in Cryptococcus neoformans (Dumesic et al. 2015), the role of facultative heterochromatin-mediated gene silencing is no longer required for proper development. Intriguingly, these two categories are not congruent with the fungal evolution history. It does not correlate either with DNA methylation contents. Furthermore, abnormal development of Kmt1/HP1 mutants in a panel of fungi, including $N$. crassa (Table 3), may point toward a transfer of reversible gene silencing function from facultative to constitutive heterochromatin. Previous studies already suggested that the ancestral role of Enhancer-of-zeste enzymes, along with the SET-domain SU(VAR)3-9 enzymes may be to 
silence TEs, through the concerted action of both H3K27me3 and H3K9me3 (Frapporti et al. 2019; Montgomery et al. 2020; Veluchamy et al. 2015). The frequent overlap of these two histone modifications we uncovered in $P$. anserina's chromatin could be a relic of this genome defense procedure. Under this hypothesis, specialization of the Enhancer-of-zeste proteins toward developmental and lineage-specific roles may have evolved latter on during evolution or may have been lost as in Mucor, Rhizopus and Aspergillus (Freitag 2017). What makes PCR2 machinery dispensable for some fungi while it is mandatory to achieve their life cycle for others? What are the evolutionary drives that selected for PCR2-dependant versus PCR2-independent regulation of gene expression? These questions remain to be further addressed. Moreover, in fungi showing a conserved function of PRC2, the PRC1 complex is not present. This implies that if H3K27me still serves as a repressive mark, alternative unknown mechanisms should replace the downstream PRC1 readers, although its $\mathrm{H} 2 \mathrm{~A}$ mono-ubiquitylation activity is not always required for silencing (Pengelly et al. 2015).

Altogether, our findings using $P$. anserina as model organism shed new light on interactions of facultative and constitutive heterochromatin in eukaryotes. 


\section{Materials and Methods}

\section{Strains and culture conditions}

The strains used in this study derived from the " $S$ " wild-type strain of $P$. anserina that was used for sequencing (Espagne et al. 2008; Grognet, Bidard, et al. 2014). Standard culture conditions, media and genetic methods for $P$. anserina have been described (Rizet and Engelmann 1949; Philippe Silar 2013) and the most recent protocols can be accessed at http://podospora.i2bc.paris-saclay.fr. Mycelium growth is performed on M2 minimal medium, in which carbon is supplied as dextrin and nitrogen as urea. Ascospores germinate on a specific germination medium ( $G$ medium) containing ammonium acetate. The methods used for nucleic acid extraction and manipulation have been described

(Ausubel et al. 1987; Lecellier and Silar 1994). Transformations of $P$. anserina protoplasts were carried out as described previously (Brygoo and Debuchy 1985).

\section{Identification and deletions of the PaKmt1, PaKmt6 and PaHP1 genes}

PaKmt1, PaKmt6 and PaHP1 genes were identified by searching the complete genome of $P$. anserina with tblastn (Altschul et al. 1990), using the N. crassa proteins DIM-5 (NCU04402), SET-7 (NCU07496) and HP1 (NCU04017). We identified three genes: Pa_6_990, Pa_1_6940 and Pa_4_7200 renamed PaKmt1, PaKmt6 and PaHP1 respectively. To confirm gene annotation, PaKmt1 transcripts were amplified by RT-PCR experiments performed on total RNA extracted from growing mycelium (1-day- and 4-day-old mycelium) and developing fruiting bodies ( 2 and 4 days post-fertilization) using primers 5s-DIM5 and 3s-DIM5 (Table S4A). Functional annotation was performed using InterProScan analysis (http://www.ebi.ac.uk/interpro/search/sequence-search), Panther Classification System (http://www.pantherdb.org/panther/), PFAM (http://pfam.xfam.org/) and Prosite (http://prosite.expasy.org/).

Deletions were performed on a $\Delta$ mus $51::$ bleoR strain lacking the mus-51 subunit of the complex involved in end-joining of DNA double strand breaks as described in (Lambou et al. 2008). In this strain, DNA integration mainly proceeds through homologous recombination. 
Replacement of the PaKmt1 wild-type allele with hygromycin-B resistance marker generated viable mutants carrying the $\Delta$ PaKmt1 null allele. Replacement of the PaKmt6 wild-type allele with nourseothricin resistance marker generated viable but severely impaired mutants. Replacement of the PaHP1 wild-type allele with hygromycin-B resistance marker also generated viable mutants carrying the $\triangle P a H P 1$ null allele. All deletions were verified by Southern blot (Fig. S5 and Fig S12A).

Constructing of the fluorescent-HA-tagged chimeric proteins PaHP1-GFP-HA, PaKmt1mCherry-HA, PaKmt6-GFP-HA.

The pAKS106 and pAKS120 plasmids were described in (Grognet et al. 2019). All the cloned inserts were sequenced before transformation.

To gain insight into PaHP1 localization, we constructed a PaHP1-GFP-HA fusion chimeric protein. To this end, PaHP1 gene was PCR amplified from the S strain genomic DNA using primers FC3-BamH1 and FC4-GFP (Table S4A). The resulting amplicon harbors 520 bp of promoter sequence followed by PaHP1 CDS cleared from its stop codon. In parallel, eGFP CDS was amplified from peGFP plasmid using FC5-HP1 and FC6-HindIII primers. The two DNA segments were fused by PCR using primers FC3-BamH1 and FC6-BamH1. This chimeric DNA fragment was digested with BamHI and Hindll and cloned into the pAKS106 plasmid linearized with the same enzymes. When transformed into $\triangle P a H P 1$ mutant strain, the PaHP1-GFP-HA allele is able to restore a wild-type phenotype (growth rate and aerial mycelium), showing that the tagged version of PaHP1 protein is functional.

PaKmt1-mCherry-HA allele construction followed the same experimental design used for PaHP1-GFP-HA construction. First, PaKmt1 was PCR amplified using Dim5mChFBamH1 and Dim5mChR and resulted in a DNA amplicon composed of 454 bp of promoter followed by the PaKmt1 CDS. The mCherry CDS was amplified from pmCherry plasmid using mChDim5F and mChDim5RHindIII primers. Fragments were fused by PCR, digested and cloned in pAKS106. Kmt1-mCherry-HA allele was able to restore wild-type growth phenotype when transformed in $\triangle P a K m t 1$ mutant strains, suggesting that this chimeric protein is functional. 
PaKmt6 (Pa_1_6940) was PCR amplified from S genomic DNA using FC73-GA1KMT6 and FC74-GA1KMT6 primers, resulting in 4232 bp DNA amplicons composed of 912 bp of promoter followed by PaKmt6 CDS cleared from its stop codon. In parallel, GFP allele was amplified from peGFP plasmid using FC75-GA1GFP and FC76-GA1GFP. Both fragments were cloned together in one step in the pAKS-106 plasmid previously digested with Notl and BamHI restriction enzymes. This cloning step was performed using Gibson Assembly kit (New England Biolabs). This yielded the chimeric PaKmt6-GFP-HA allele. An additional PaKmt6-HA allele was generated by PCR amplification from the wild-type strain DNA of PaKmt6 CDS along with its own promoter, using FC65-Notl and FC72-BamHI primers. These amplicons were digested and cloned in pAKS106 plasmid using Notl and BamHI restriction enzymes. When transformed into $\triangle$ PaKmt6 mutant strain, both PaKmt6-GFP-HA and PaKmt6-HA alleles were able to restore wild-type growth and sexual development, suggesting that this chimeric version of the PaKmt6 protein is functional. However, no GFP fluorescence signal was detected in the $\triangle$ PaKmt6, PaKmt6-GFP-HA complemented strains.

To overexpress PaHP1, the endogenous promoter were replaced by the AS4 promoter that drives high and constitutive expression throughout the life cycle (P Silar and Picard 1994). PaHP1-GFP allele was PCR amplified from pAKS106-HP1-GFP (this study) using primers FC21-BamH1 and FC6-HindIII. The resulting 1877 bp amplicons harbor the chimeric construction cleared from its stop codon and promoter. The PCR product was digested with BamHI and Hindll and then cloned in pAKS120 previously digested with the same enzymes. The pAS4-PaHP1-GFP-HA resulting allele was introduced in $\triangle P a H P 1$ strains by transformation.

pAS4-PaKmt1-mCherry-HA over-expressed allele construction followed the same experimental procedures as the one used for pAS4-HP1-GFP-HA construction. PaKmt1mCherry CDS was PCR amplified using FC22-BamHI and mChDim5R-Hindll using pAKS106-PaKmt1-mCherry-HA plasmid as DNA template. Amplified DNA fragments were then digested and cloned in pAKS120 plasmid using HindllI and BamHI restriction enzymes. This allele was transformed in $\triangle P a K m t 1$ strain. 


\section{Complementation experiments with GFP-tagged proteins}

To perform complementation experiments, PaKmt1-mCherry allele was transformed into the $\triangle$ PaKmt1 strain. Because PaKmt1-mCherry allele was linked to a phleomycin marker, 55 phleomycin resistant transformants were recovered. Among those, five wild-type looking independent transgenic strains were selected and crossed to a wild-type strain. In the progeny, all the phleomycin and hygromycin resistant haploid strains behaved similarly to wild-type strain. Two of these independent transformants were selected for further phenotypic characterization. To ask whether a constitutively over-expressed PaKmt1 allele could have an impact on the physiology of $P$. anserina, we introduced the AS4-PaKmt1mCherry allele into the $\Delta P a K m t 1$ strain. All the phleomycin resistant transformants looked like a wild-type strain ( $\mathrm{N}=30$ phleomycin resistant). Two of them were crossed to a wild-type strain. In their progeny, all the phleomycin resistant strains carrying either the PaKmt1+ allele or the $\Delta$ PaKmt1 were indistinguishable from the wild-type strains.

Similarly, we transformed a PaHP1-GFP-HA allele, linked to a phleomycin marker, into a mutant $\triangle P a H P 1$ strain. Among the 20 phleomycin resistant transformants that were recovered, five independent transformants displaying a wild-type phenotype were selected and crossed to the wild-type strain. In the progeny, all the phleomycin and hygromycin resistant haploid strains behaved similarly to the wild-type strain. Transformation of the AS4PaHP1-GFP-HA allele into the $\triangle P a H P 1$ strain generated phleomycin resistant transformants $(\mathrm{N}=19)$ showing intermediate to full complementation but no extra phenotypes. Two transformants presenting full complementation were crossed to a wild-type strain. In their progeny, all the phleomycin resistant strains carrying either the $\mathrm{PaHP}^{+}$allele or the $\triangle \mathrm{PaHP1}$ behaved like the wild-type strains.

Finally, the PaKmt6-HA allele and the PaKmt6-GFP-HA allele were independently transformed into a $\Delta P a K m t 6$ strain. For the two transformation experiments, among the phleomycin resistant transformants that were recovered ( $\mathrm{N}=20$ and $\mathrm{N}=32$, respectively), two independent transgenic strains displaying a wild-type phenotype were selected and crossed 
to a wild-type strain. In the progeny of these four crosses, all the phleomycin and nourseothricin resistant haploid strains behaved similarly to wild-type strain.

\section{Phylogenetic analysis}

Orthologous genes were identified using the MycoCosm portal (Grigoriev et al. 2014) and manually verified by reciprocal Best Hit BLAST analysis. Sequences were aligned using Muscle (http://www.ebi.ac.uk/Tools/msa/muscle/) and trimmed using Jalview (version 2.9.0b2) to remove non-informative regions (i.e. poorly aligned regions and/or gaps containing regions). Trees were constructed with PhyML 3.0 software with default parameters and 100 bootstrapped data set (Guindon et al. 2010). The trees were visualized with the iTOL server (http://itol.embl.de/).

\section{Phenotypic analyses}

Spermatium counting was performed as follows: each strain was grown on M2 medium with two cellophane layers at $18^{\circ} \mathrm{C}$ for 14 days (P. Silar's personal communication). To collect spermatia, cultures were washed with $1.5 \mathrm{~mL}$ of sterile water. Numeration proceeded through Malassez counting chamber.

\section{Cytological analysis}

Light microscopy was performed on two-day-old mycelium. Explants taken from complemented strains expressing either PaHP1-GFP-HA or PaKmt1-mCherry-HA were analyzed by fluorescence microscopy. Pictures were taken with a Leica DMIRE 2 microscope coupled with a 10-MHz Cool SNAPHQ charge-coupled device camera (Roper Instruments), with a z-optical spacing of $0.5 \mathrm{~mm}$. The GFP filter was the GFP-3035B from Semrock (Ex: 472nm/30, dichroïc: 495nm, Em: 520nm/35). The Metamorph software (Universal Imaging Corp.) was used to acquire z-series. Images were processed using the ImageJ software (NIH, Bethesda).

\section{Western blot analysis}

Western blots were performed on nuclear extracts made of $5.10^{7}$ protoplasts resuspended in $3 \mathrm{~mL}$ of New Cell Lysis Buffer (Tris-HCL pH 7.550 mM, NaCl 150 mM, EDTA 5 mM, NP-40 $0.5 \%$, Triton $1 \%$ and protease inhibitor (Roche-04693132001). The nuclear extracts were 
collected after centrifugation (300 g, $5 \mathrm{~min}$ ) and resuspended in $300 \mu \mathrm{L}$ of Nuclei Lysis Buffer (Tris- $\mathrm{HCl}$ pH $850 \mathrm{mM}$, EDTA $10 \mathrm{mM}$, SDS 0.5\% and protease inhibitor (Roche04693132001). Nuclei were then sonicated (Diagenode bioruptor plus sonicatore, $20 \mathrm{~min}$ at high level). Protein contents of nuclear extracts were quantified using the Qubit Protein assay kit (Invitrogen). Then, $35 \mu \mathrm{g}$ of nuclear extract were diluted in Laemmli sample buffer and loaded on a $10 \%$ SDS polyacrylamide gel. Membranes were probed using the following high affinity ChIP-grade monoclonal antibodies: anti-Histone 3 (Abcam ab12079), anti-H3K9me3 (Abcam ab8898), anti-H3K27me3 (Millipore 17-622) and anti-H3K4me3 (Active Motif 39159).

\section{Chromatin immuno-precipitation}

Each ChIP-seq experiment was performed on two independent biological replicates for each genotype (mat+ strains only). Chromatin extractions were made from mycelium grown for 60 $\mathrm{h}$ at $27^{\circ} \mathrm{C}$ in liquid culture $\left(\mathrm{KH}_{2} \mathrm{PO}_{4} 0.25 \mathrm{~g} / \mathrm{L}, \mathrm{K}_{2} \mathrm{HPO}_{4} 0.3 \mathrm{~g} / \mathrm{L}, \mathrm{MgSO}_{4} 0.25 \mathrm{~g} / \mathrm{L}\right.$, urea $0.5 \mathrm{~g} / \mathrm{L}$, biotin $0.05 \mathrm{mg} / \mathrm{L}$, thiamine $0.05 \mathrm{mg} / \mathrm{L}$, oligo-element $0.1 \%$, yeast extract $5 \mathrm{~g} / \mathrm{L}$ and dextrin 5.5 g/L). Mycelium was filtered and quickly washed with phosphate-buffered saline (PBS), resuspended and then cross-linked in $100 \mathrm{~mL}$ of PBS with $1 \%$ paraformaldehyde for 15 min at $27^{\circ} \mathrm{C}$. Reaction was stopped by addition of glycine $(60 \mathrm{mM})$. Cross-linked mycelium was filtered, washed with cold PBS buffer and dried on Whatman paper. Dried mycelium was frozen in liquid nitrogen and ground to powder in a mortar. Aliquots of mycelium powder (200 $\mathrm{mg}$ ) were resuspended in $1 \mathrm{~mL}$ of Lysis Buffer (Hepes pH $7.550 \mathrm{mM}, \mathrm{NaCl} 0.5 \mathrm{M}$, EDTA pH $81 \mathrm{mM}$, Triton X-100 1\%, Sodium deoxycholate $0.1 \%, \mathrm{CaCl}_{2} 2 \mathrm{mM}$ and protease inhibitor (Roche)). Chromatin was sheared to $0.15-0.6-k b$ fragments using Micrococcal nuclease (NEB \#M0247) for $30 \mathrm{~min}$ at $37^{\circ} \mathrm{C}(10000$ gels units $/ \mathrm{mL})$. The enzymatic digestion was stopped by addition of EGTA (15 mM). After centrifugation (15000 g, $15 \mathrm{~min}$, twice) of the digested samples, pelleted genomic DNA fragments were discarded. Concentration of the soluble chromatin fractions was assayed using the fluorescence system Qubit dsDNA HS assay kit (Invitrogen). Prior to immunoprecipitation, $5 \mu \mathrm{g}$ of soluble chromatin was washed in $1.1 \mathrm{~mL}$ of Lysis buffer containing $30 \mu \mathrm{L}$ of magnetic beads (Invitrogen $10001 \mathrm{D}, 4 \mathrm{~h}$ at $4^{\circ} \mathrm{C}$ ). From the $1.1 \mathrm{~mL}$ washed soluble chromatin cleared from magnetic beads, $100 \mu \mathrm{L}$ was kept 
for non-IP input control. Specific antibodies were added individually to the remaining $1 \mathrm{~mL}$ of the washed soluble chromatin (IP samples) for an overnight incubation at $4^{\circ} \mathrm{C}$ on a rotating wheel. Antibodies used in this study are anti-H3K9me3 (Abcam ab8898), anti-H3K27me3 (Millipore 17-622) and anti-H3K4me3 (Active Motif 39159). One additional mock sample was done with an anti-GFP antibody (Abcam ab290) in the wild-type strain only. Chromatin was IPed by adding $20 \mu \mathrm{L}$ of magnetic beads, incubated for $4 \mathrm{~h}$ at $4^{\circ} \mathrm{C}$ on a rotating wheel. Beads were successively washed in $1 \mathrm{~mL}$ for $10 \mathrm{~min}$ in of the following buffers: Lysis buffer (twice), Lysis buffer plus $0.5 \mathrm{M} \mathrm{NaCl}$, LiCl Wash Buffer (Tris-Hcl pH 810 mM, LiCl 250 mM, NP40 0.5\%, Sodium deoxycholate $0.5 \%$, EDTA pH $81 \mathrm{mM}$; twice for H3K9me3 immunoprecipitation) and finally Tris-EDTA Buffer (Tris-Hcl pH 7.510 mM, EDTA pH 81 mM). Elution of IP samples was done with TES buffer (Tris-Hcl pH 850 mM, EDTA pH 810 $\mathrm{mM}$ with SDS $1 \%$ ) at $65^{\circ} \mathrm{C}$. TES buffer was also added to non-IPed input sample. Both IP and input samples were uncrosslinked at $65^{\circ} \mathrm{C}$ overnight and treated successively with RNAse A (Thermo Fisher Scientific) $\left(0.2 \mathrm{mg} / \mathrm{mL}, 2 \mathrm{~h}\right.$ at $\left.50^{\circ} \mathrm{C}\right)$ and Proteinase $\mathrm{K}$ (Thermo Fisher Scientific) $\left(0.8 \mathrm{mg} / \mathrm{mL}, 2 \mathrm{~h}\right.$ at $\left.50^{\circ} \mathrm{C}\right)$. DNA was then subjected to phenol-chloroform purification and ethanol precipitation. DNA pellets were resuspended in $30 \mu \mathrm{L}$ of Tris-EDTA buffer.

\section{RT-qPCR experiments}

Cultures for RNA extractions were performed for $60 \mathrm{~h}$ at $27^{\circ} \mathrm{C}$ in liquid culture $\left(\mathrm{KH}_{2} \mathrm{PO}_{4} 0.25\right.$ $\mathrm{g} / \mathrm{L}, \mathrm{K}_{2} \mathrm{HPO}_{4} 0.3 \mathrm{~g} / \mathrm{L}, \mathrm{MgSO}_{4} 0.25 \mathrm{~g} / \mathrm{L}$, urea $0.5 \mathrm{~g} / \mathrm{L}$, biotin $0.05 \mathrm{mg} / \mathrm{L}$, thiamine $0.05 \mathrm{mg} / \mathrm{L}$, oligo-element $0.1 \%$, yeast extract $5 \mathrm{~g} / \mathrm{L}$ and dextrin $5.5 \mathrm{~g} / \mathrm{L}$ ). For each replicate, $100 \mathrm{mg}$ of mycelium was harvested, flash-frozen in liquid nitrogen and ground with a MikroDismembrator S (Sartorius, Göttingen, Germany) for one minute at $2600 \mathrm{rpm}$ in a Nalgene Cryogenic vial (ref \# 5000-0012, ThermoFischer Scientific, Waltham, USA) with a chromium steel grinding ball (ref \# BBI-8546916, Sartorius, Göttingen, Germany). Total RNAs were extracted with the RNeasy Plant Mini Kit (ref \# 74904, Qiagen, Hilden, Germany), according to the protocol described for Plants and Fungi with buffer RLT. Contaminating DNA was digested in RNA solutions with RNase-free DNase (ref \# 79254, Qiagen, Hilden) and RNAs 
were purified once more with the RNeasy Plant Mini Kit (ref \# 74904, Qiagen, Hilden, Germany), according to the protocol described for RNA cleanup. RNAs were quantified with a DeNovix DS-11 spectrophotometer (Willmington, USA), checked for correct 260/280 and 260/230 ratios, and RNA quality was checked by gel electrophoresis. Reverse transcription was performed with the SuperScript III Reverse Transcriptase (ref \# 18080093, Invitrogen, Carlsbad, USA) and oligo-dT. A non-reverse transcribed (NRT) control was systematically performed on a pool of NRT controls to ensure that the Cq was above the Cq obtained from corresponding reverse transcribed RNAs. For genes without introns, NRT control was performed for each replicate sample to ensure that the $\mathrm{Cq}$ was above the $\mathrm{Cq}$ obtained from corresponding reverse transcribed RNAs. Experiments were run with five biological replicates for each strain, and each biological replicate was run in technical triplicates (see Table S3 for Cq), except NRT control which were in technical duplicates (see Table S3 for Cq). When possible, primers were designed on two consecutive exons (Table S4B). Normalization genes were selected among a set of 8 housekeeping genes using geNorm (see Table S3 for details of analysis) (Vandesompele et al. 2002). RT-qPCR normalization according to the $\Delta \Delta \mathrm{Ct}$ method (Pfaffl, Horgan, and Dempfle 2002), standard error and $95 \%$ confidence interval calculations, and statistical analyses were performed using REST 2009 software (Qiagen, Hilden, Germany). Genes were defined as downregulated in the mutant strain if the ratio of their transcript level in the mutant strain compared with that in the wildtype strain showed a-fold change $>0$ and $<1$, with a $p$-value $<0.05$. On the other hand, genes were defined as up-regulated in the mutant strain if the ratio of their transcript level in the mutant strain compared with that in the wild-type strain showed a $>1$-fold change, with a p-value $<0.05$. Ratios with a $95 \%$ confidence interval, including 1 , were not considered significant (du Prel et al. 2009). RT-qPCR experiments were MIQE compliant (Bustin et al. 2009).

\section{qPCR Analysis to test ChIP efficency}

Quantitative PCR experiments were performed with dsDNA fluorescent detection method using the FastStart Universal SYBR Green Master from Roche (04913850001). 
IP enrichments were assayed with the following protocol: $5 \mu \mathrm{L}$ of IPed DNA samples and non-IPed input were diluted 10 times in nuclease free water. qPCR experiments were done on $2 \mu \mathrm{L}$ of the diluted samples using the pairs of primers $(0.5 \mu \mathrm{M})$ FC77-Actin/FC78-Actin and FC125-580/FC126-580 (see Table S4B). For each IP sample, relative concentrations were determined as an "Input percentage" with the following equation:

$$
\% \text { Input }=\left(\mathrm{E}\left(\text { CqInput }-\left(\frac{\ln (10)}{\ln E}\right)\right)-\text { CqSample }\right) \times 100
$$

\section{Sequencing}

ChIP-seq libraries were built using the NEB Next Ultrall DNA library Prep kit for Illumina (New England Biolabs \#E7645S/L) and Agencourt Ampure XP beads (Beckman Coulter, \#A63880) according to the supplier protocols. PCR amplification was made of 12 cycles. ChIP-sequencing was performed by the I2BC High-throughput sequencing facility (NextSeq 500/550 High Output Kit v2 (75 cycles), SR 75 bp). Reads were trimmed with Cutadapt 1.15 and filtered for control quality by FastQC v0.11.5.

\section{ChIP-seq sequencing data analysis}

Sequencing generated between 8 and 45 million reads depending on samples. The ChIPseq analysis was performed on sets of data that contained 8 to 10 million reads at most. If needed, the number of reads was down-sized randomly. Reads were mapped on the S mat+ P. anserina genome (Espagne et al. 2008) using Bowtie 2 software (version 2.3.0, see annex for results). For visualization purpose, data were normalized using Deeptools 2.0 (Ramírez et al. 2016) bamcoverage with the BPM method. Spearman correlation factors were calculated on normalized data using Deeptools 2.0. For each condition, we made sure that the two biological replicates correlate (Fig. S14 and Table S6 and S7) and then merge them for peak calling. Peak calling was performed using MACS2 software (version 2.1.1) using the mock sample as control. Further peaks annotations and comparisons were done using Bedtools (version 2.26.0). MACS2 predicted peaks have been mapped on the genome using the ChromoMap R package (v. 0.2, https://cran.r-project.org/package=chromoMap). Genome- 
wide visualization of peaks was also generated with the Circos software (Krzywinski et al. 2009). Box plots have been drawn using ggplot2 (Wickham 2009). All the over plots have been generated using Deeptools2. Genomic regions have been extracted from the current genome annotation. Promoters have been arbitrarily defined as $1 \mathrm{~kb}$ in $5^{\prime}$ of the start codon. Scores of the heatmap represent the mapped reads after normalization, hence not only the MACS2 predicted peaks. The Euler diagram has been generated by the eulerr $\mathrm{R}$ package ( $\mathrm{V}$ 6.0.0, https://cran.r-project.org/package=eulerr).

\section{Availability of data and materials' statement}

The datasets generated and/or analyzed during the current study are available in the NCBI

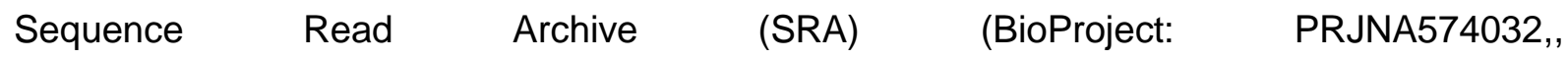
https://dataview.ncbi.nlm.nih.gov/object/PRJNA574032?reviewer=7beuetatcp00b0g33k3vqsn o59).

\section{Competing interests}

The authors declare that they have no competing interests.

\section{Funding}

FC was recipient of a LIDEX BIG Paris-Saclay Ph.D. fellowship. P.G., F.C. and F.M. were supported by grants from UMR9198.

\section{Authors' contributions}

Conceived and designed the experiments: FM. Performed the experiments: FC, VC, RD, LM, FM. Analyzed the data: FC, RD, DN, PG, FM. Contributed reagents/materials/analysis tools/expertise sharing: PG, CS, DN. Wrote the paper: FC, DN, PG, FM. 


\section{Acknowledgments}

We acknowledge the technical assistance of Sylvie François. We acknowledge the highthroughput sequencing facility of I2BC for its sequencing and bioinformatics expertise. We are thankful to Sébastien Bloyer for providing expertise to start the project as well as for fruitful scientific discussions. We thank Gaëlle Lelandais for help with statistical analysis. 


\section{References}

Alder, Olivia, Fabrice Lavial, Anne Helness, Emily Brookes, Sandra Pinho, Anil Chandrashekran, Philippe Arnaud, Ana Pombo, Laura O'Neill, and Véronique Azuara. 2010. "Ring1B and Suv39h1 Delineate Distinct Chromatin States at Bivalent Genes during Early Mouse Lineage Commitment." Development (Cambridge, England) 137 (15): 2483-92. https://doi.org/10.1242/dev.048363.

Altschul, S F, W Gish, W Miller, E W Myers, and D J Lipman. 1990. "Basic Local Alignment Search Tool.” Journal of Molecular Biology 215 (3): 403-10. https://doi.org/10.1006/jmbi.1990.9999.

Angel, Andrew, Jie Song, Caroline Dean, and Martin Howard. 2011. "A Polycomb-Based Switch Underlying Quantitative Epigenetic Memory." Nature 476 (7358): 105-8.

https://doi.org/10.1038/nature10241.

Ausubel, FM, R Brent, RE Kingston, DD Moore, JG Seidman, JA Smith, and K Struhl. 1987. Current Protocols in Molecular Biology. Wiley Interscience. New York.

Basenko, Evelina Y., Takahiko Sasaki, Lexiang Ji, Cameron J. Prybol, Rachel M. Burckhardt, Robert J. Schmitz, and Zachary A. Lewis. 2015. "Genome-Wide Redistribution of H3K27me3 Is Linked to Genotoxic Stress and Defective Growth." Proceedings of the National Academy of Sciences of the United States of America 112 (46): E6339-6348. https://doi.org/10.1073/pnas.1511377112.

Becker, Justin S., Dario Nicetto, and Kenneth S. Zaret. 2016. "H3K9me3-Dependent Heterochromatin: Barrier to Cell Fate Changes." Trends in Genetics: TIG 32 (1): 29-41. https://doi.org/10.1016/j.tig.2015.11.001.

Bhaumik, Sukesh R., Edwin Smith, and Ali Shilatifard. 2007. "Covalent Modifications of Histones during Development and Disease Pathogenesis." Nature Structural \& Molecular Biology 14 (11): 1008-16. https://doi.org/10.1038/nsmb1337.

Birve, A., A. K. Sengupta, D. Beuchle, J. Larsson, J. A. Kennison, null Rasmuson-Lestander A, and J. Müller. 2001. "Su(z)12, a Novel Drosophila Polycomb Group Gene That Is Conserved in Vertebrates and Plants." Development (Cambridge, England) 128 (17): 3371-79.

Boivin, Antoine, Sébastien Gaumer, and Annie Sainsard-Chanet. 2008. "Life Span Extension by Dietary Restriction Is Reduced but Not Abolished by Loss of Both SIR2 and HST2 in Podospora anserina." Mechanisms of Ageing and Development 129 (12): 714-21.

https://doi.org/10.1016/j.mad.2008.09.011.

Brygoo, Y, and R Debuchy. 1985. "Transformation by Integration in Podospora anserina. I. Methodology and Phenomenology." 200: 128-31.

Bustin, Stephen A., Vladimir Benes, Jeremy A. Garson, Jan Hellemans, Jim Huggett, Mikael Kubista, Reinhold Mueller, et al. 2009. "The MIQE Guidelines: Minimum Information for Publication of Quantitative Real-Time PCR Experiments." Clinical Chemistry 55 (4): 611-22.

https://doi.org/10.1373/clinchem.2008.112797.

Canzio, Daniele, Evelyn Y. Chang, Smita Shankar, Kristopher M. Kuchenbecker, Matthew D. Simon, Hiten D. Madhani, Geeta J. Narlikar, and Bassem Al-Sady. 2011. "Chromodomain-Mediated

Oligomerization of HP1 Suggests a Nucleosome-Bridging Mechanism for Heterochromatin Assembly." Molecular Cell 41 (1): 67-81. https://doi.org/10.1016/j.molcel.2010.12.016.

Chujo, Tetsuya, and Barry Scott. 2014a. "Histone H3K9 and H3K27 Methylation Regulates Fungal Alkaloid Biosynthesis in a Fungal Endophyte-Plant Symbiosis." Molecular Microbiology 92 (2): 413-34. https://doi.org/10.1111/mmi.12567.

Connolly, Lanelle R., Kristina M. Smith, and Michael Freitag. 2013. "The Fusarium graminearum Histone H3 K27 Methyltransferase KMT6 Regulates Development and Expression of Secondary Metabolite Gene Clusters." PLoS Genetics 9 (10): e1003916.

https://doi.org/10.1371/journal.pgen.1003916.

Conway, Eric, Evan Healy, and Adrian P. Bracken. 2015. "PRC2 Mediated H3K27 Methylations in Cellular Identity and Cancer." Current Opinion in Cell Biology 37 (December): 42-48.

https://doi.org/10.1016/j.ceb.2015.10.003.

Coppin, E., S. Arnaise, V. Contamine, and M. Picard. 1993. "Deletion of the Mating-Type Sequences in Podospora anserina Abolishes Mating without Affecting Vegetative Functions and Sexual Differentiation." Molecular \& General Genetics: MGG 241 (3-4): 409-14. 
Coppin, Evelyne, and Philippe Silar. 2007. "Identification of PaPKS1, a Polyketide Synthase Involved in Melanin Formation and Its Use as a Genetic Tool in Podospora anserina." Mycological Research 111 (Pt 8): 901-8. https://doi.org/10.1016/j.mycres.2007.05.011.

Deleris, Angelique, Hume Stroud, Yana Bernatavichute, Elizabeth Johnson, Gregor Klein, Daniel Schubert, and Steven E. Jacobsen. 2012. "Loss of the DNA Methyltransferase MET1 Induces H3K9 Hypermethylation at PcG Target Genes and Redistribution of H3K27 Trimethylation to Transposons in Arabidopsis thaliana." PLoS Genetics 8 (11): e1003062. https://doi.org/10.1371/journal.pgen.1003062.

Dumesic, Phillip A., Christina M. Homer, James J. Moresco, Lindsey R. Pack, Erin K. Shanle, Scott M. Coyle, Brian D. Strahl, Danica G. Fujimori, John R. Yates, and Hiten D. Madhani. 2015. "Product Binding Enforces the Genomic Specificity of a Yeast Polycomb Repressive Complex." Cell 160 (1-2): 204-18. https://doi.org/10.1016/j.cell.2014.11.039.

Eissenberg, J. C., T. C. James, D. M. Foster-Hartnett, T. Hartnett, V. Ngan, and S. C. Elgin. 1990. "Mutation in a Heterochromatin-Specific Chromosomal Protein Is Associated with Suppression of Position-Effect Variegation in Drosophila melanogaster." Proceedings of the National Academy of Sciences of the United States of America 87 (24): 9923-27. https://doi.org/10.1073/pnas.87.24.9923.

Espagne, Eric, Olivier Lespinet, Fabienne Malagnac, Corinne Da Silva, Olivier Jaillon, Betina M Porcel, Arnaud Couloux, et al. 2008. "The Genome Sequence of the Model Ascomycete Fungus Podospora anserina." Genome Biology 9 (5): R77. https://doi.org/10.1186/gb-2008-9-5-r77.

Frapporti, Andrea, Caridad Miró Pina, Olivier Arnaiz, Daniel Holoch, Takayuki Kawaguchi, Adeline Humbert, Evangelia Eleftheriou, et al. 2019. "The Polycomb Protein Ezl1 Mediates H3K9 and H3K27 Methylation to Repress Transposable Elements in Paramecium." Nature Communications 10 (June). https://doi.org/10.1038/s41467-019-10648-5.

Freitag, Michael. 2017. "Histone Methylation by SET Domain Proteins in Fungi." Annual Review of Microbiology 71: 413-39. https://doi.org/10.1146/annurev-micro-102215-095757.

Freitag, Michael, Patrick C. Hickey, Tamir K. Khlafallah, Nick D. Read, and Eric U. Selker. 2004. "HP1 Is Essential for DNA Methylation in Neurospora." Molecular Cell 13 (3): 427-34. DOI: 10.1016/s10972765(04)00024-3.

Freitag, Michael, Rebecca L Williams, Gregory O Kothe, and Eric U Selker. 2002. "A Cytosine Methyltransferase Homologue Is Essential for Repeat-Induced Point Mutation in Neurospora crassa." Proceedings of the National Academy of Sciences of the United States of America 99 (13): 8802-7. https://doi.org/10.1073/pnas.132212899.

Gacek, Agnieszka, and Joseph Strauss. 2012. "The Chromatin Code of Fungal Secondary Metabolite Gene Clusters." Applied Microbiology and Biotechnology 95 (6): 1389-1404. https://doi.org/10.1007/s00253-012-4208-8.

Gibson, William T., Rebecca L. Hood, Shing Hei Zhan, Dennis E. Bulman, Anthony P. Fejes, Richard Moore, Andrew J. Mungall, et al. 2012. "Mutations in EZH2 Cause Weaver Syndrome." American Journal of Human Genetics 90 (1): 110-18. https://doi.org/10.1016/j.ajhg.2011.11.018.

Goodrich, J., P. Puangsomlee, M. Martin, D. Long, E. M. Meyerowitz, and G. Coupland. 1997. "A Polycomb-Group Gene Regulates Homeotic Gene Expression in Arabidopsis." Nature 386 (6620): 4451. https://doi.org/10.1038/386044a0.

Grigoriev, Igor V., Roman Nikitin, Sajeet Haridas, Alan Kuo, Robin Ohm, Robert Otillar, Robert Riley, et al. 2014. "MycoCosm Portal: Gearing up for 1000 Fungal Genomes." Nucleic Acids Research 42 (Database issue): D699-704. https://doi.org/10.1093/nar/gkt1183.

Grognet, Pierre, Frédérique Bidard, Claire Kuchly, Laetitia Chan Ho Tong, Evelyne Coppin, Jinane Ait Benkhali, Arnaud Couloux, Patrick Wincker, Robert Debuchy, and Philippe Silar. 2014. "Maintaining Two Mating Types: Structure of the Mating Type Locus and Its Role in Heterokaryosis in Podospora anserina." Genetics 197 (1): 421-32. https://doi.org/10.1534/genetics.113.159988.

Grognet, Pierre, Hervé Lalucque, Fabienne Malagnac, and Philippe Silar. 2014. "Genes That Bias Mendelian Segregation." PLoS Genetics 10 (5): e1004387.

https://doi.org/10.1371/journal.pgen.1004387.

Grognet, Pierre, Hélène Timpano, Florian Carlier, Jinane Aït-Benkhali, Véronique Berteaux-Lecellier, Robert Debuchy, Frédérique Bidard, and Fabienne Malagnac. 2019. "A RID-like Putative Cytosine Methyltransferase Homologue Controls Sexual Development in the Fungus Podospora anserina." PLoS Genetics 15 (8): e1008086. https://doi.org/10.1371/journal.pgen.1008086. 
Gu, Qin, Tiantian Ji, Xiao Sun, Hai Huang, Hao Zhang, Xi Lu, Liming Wu, Rong Huo, Huijun Wu, and Xuewen Gao. 2017. "Histone H3 Lysine 9 Methyltransferase FvDim5 Regulates Fungal Development, Pathogenicity and Osmotic Stress Responses in Fusarium verticillioides." FEMS Microbiology Letters 364 (19). https://doi.org/10.1093/femsle/fnx184.

Guindon, Stéphane, Jean-François Dufayard, Vincent Lefort, Maria Anisimova, Wim Hordijk, and Olivier Gascuel. 2010. "New Algorithms and Methods to Estimate Maximum-Likelihood Phylogenies: Assessing the Performance of PhyML 3.0." Systematic Biology 59 (3): 307-21. https://doi.org/10.1093/sysbio/syq010.

Ho, Joshua W. K., Youngsook L. Jung, Tao Liu, Burak H. Alver, Soohyun Lee, Kohta Ikegami, KyungAh Sohn, et al. 2014. "Comparative Analysis of Metazoan Chromatin Organization." Nature 512 (7515): 449-52. https://doi.org/10.1038/nature13415.

Honda, Shinji, and Eric U Selker. 2008. "Direct Interaction between DNA Methyltransferase DIM-2 and HP1 Is Required for DNA Methylation in Neurospora crassa." Molecular and Cellular Biology 28 (19): 6044-55. https://doi.org/10.1128/MCB.00823-08.

James, T. C., and S. C. Elgin. 1986. "Identification of a Nonhistone Chromosomal Protein Associated with Heterochromatin in Drosophila melanogaster and Its Gene." Molecular and Cellular Biology 6 (11): 3862-72. https://doi.org/10.1128/mcb.6.11.3862.

Jamet-Vierny, Corinne, Robert Debuchy, Magali Prigent, and Philippe Silar. 2007. “IDC1, a Pezizomycotina-Specific Gene That Belongs to the PaMpk1 MAP Kinase Transduction Cascade of the Filamentous Fungus Podospora anserina." Fungal Genetics and Biology: FG \& B 44 (12): 1219-30. https://doi.org/10.1016/j.fgb.2007.04.005.

Jamieson, Kirsty, Michael R. Rountree, Zachary A. Lewis, Jason E. Stajich, and Eric U. Selker. 2013. "Regional Control of Histone H3 Lysine 27 Methylation in Neurospora." Proceedings of the National Academy of Sciences of the United States of America 110 (15): 6027-32. https://doi.org/10.1073/pnas.1303750110.

Jamieson, Kirsty, Elizabeth T. Wiles, Kevin J. McNaught, Simone Sidoli, Neena Leggett, Yanchun Shao, Benjamin A. Garcia, and Eric U. Selker. 2016. "Loss of HP1 Causes Depletion of H3K27me3 from Facultative Heterochromatin and Gain of H3K27me2 at Constitutive Heterochromatin." Genome Research 26 (1): 97-107. https://doi.org/10.1101/gr.194555.115.

Klocko, Andrew D., Tereza Ormsby, Jonathan M. Galazka, Neena A. Leggett, Miki Uesaka, Shinji Honda, Michael Freitag, and Eric U. Selker. 2016. "Normal Chromosome Conformation Depends on Subtelomeric Facultative Heterochromatin in Neurospora crassa." Proceedings of the National Academy of Sciences of the United States of America 113 (52): 15048-53. https://doi.org/10.1073/pnas.1615546113.

Kouzarides, Tony. 2007. "Chromatin Modifications and Their Function." Cell 128 (4): 693-705. https://doi.org/10.1016/j.cell.2007.02.005.

Kouzminova, E, and E U Selker. 2001. "Dim-2 Encodes a DNA Methyltransferase Responsible for All Known Cytosine Methylation in Neurospora." The EMBO Journal 20 (15): 4309-23. https://doi.org/10.1093/emboj/20.15.4309.

Krzywinski, Martin I., Jacqueline E. Schein, Inanc Birol, Joseph Connors, Randy Gascoyne, Doug Horsman, Steven J. Jones, and Marco A. Marra. 2009. "Circos: An Information Aesthetic for Comparative Genomics.” Genome Research, June. https://doi.org/10.1101/gr.092759.109.

Lambou, Karine, Fabienne Malagnac, Crystel Barbisan, Didier Tharreau, Marc-Henri Lebrun, and Philippe Silar. 2008. "The Crucial Role of the Pls1 Tetraspanin during Ascospore Germination in Podospora anserina Provides an Example of the Convergent Evolution of Morphogenetic Processes in Fungal Plant Pathogens and Saprobes." Eukaryotic Cell 7 (10): 1809-18. https://doi.org/10.1128/EC.00149-08.

Larson, Adam G., Daniel Elnatan, Madeline M. Keenen, Michael J. Trnka, Jonathan B. Johnston, Alma L. Burlingame, David A. Agard, Sy Redding, and Geeta J. Narlikar. 2017. "Liquid Droplet Formation by HP1a Suggests a Role for Phase Separation in Heterochromatin." Nature 547 (7662): 236-40. https://doi.org/10.1038/nature22822.

Lecellier, G, and P Silar. 1994. "Rapid Methods for Nucleic Acids Extraction from Petri Dish-Grown Mycelia." Current Genetics 25 (2): 122-23.

Lewis, E. B. 1978. “A Gene Complex Controlling Segmentation in Drosophila.” Nature 276 (5688): 
565-70. https://doi.org/10.1038/276565a0.

Li, Jiali, Ronald P. Hart, Elyse M. Mallimo, Mavis R. Swerdel, Alexander W. Kusnecov, and Karl Herrup. 2013. "EZH2-Mediated H3K27 Trimethylation Mediates Neurodegeneration in AtaxiaTelangiectasia." Nature Neuroscience 16 (12): 1745-53. https://doi.org/10.1038/nn.3564.

Liu, Sheng, Julie Brind'Amour, Mohammad M. Karimi, Kenjiro Shirane, Aaron Bogutz, Louis Lefebvre, Hiroyuki Sasaki, Yoichi Shinkai, and Matthew C. Lorincz. 2014. "Setdb1 Is Required for Germline Development and Silencing of H3K9me3-Marked Endogenous Retroviruses in Primordial Germ Cells." Genes \& Development 28 (18): 2041-55. https://doi.org/10.1101/gad.244848.114.

Majewski, lan J., Marnie E. Blewitt, Carolyn A. de Graaf, Edward J. McManus, Melanie Bahlo, Adrienne A. Hilton, Craig D. Hyland, et al. 2008. "Polycomb Repressive Complex 2 (PRC2) Restricts Hematopoietic Stem Cell Activity." PLoS Biology 6 (4): e93. https://doi.org/10.1371/journal.pbio.0060093.

Malagnac, F, H Lalucque, G Lepère, and P Silar. 2004. "Two NADPH Oxidase Isoforms Are Required for Sexual Reproduction and Ascospore Germination in the Filamentous Fungus Podospora anserina." Fungal Genetics and Biology: FG \& B 41 (11): 982-97. https://doi.org/10.1016/j.fgb.2004.07.008.

Martens, Joost H. A., Roderick J. O'Sullivan, Ulrich Braunschweig, Susanne Opravil, Martin Radolf, Peter Steinlein, and Thomas Jenuwein. 2005. "The Profile of Repeat-Associated Histone Lysine Methylation States in the Mouse Epigenome." The EMBO Journal 24 (4): 800-812. https://doi.org/10.1038/sj.emboj.7600545.

Möller, Mareike, Klaas Schotanus, Jessica L. Soyer, Janine Haueisen, Kathrin Happ, Maja Stralucke, Petra Happel, et al. 2019. "Destabilization of Chromosome Structure by Histone H3 Lysine 27 Methylation." PLoS Genetics 15 (4): e1008093. https://doi.org/10.1371/journal.pgen.1008093.

Money, Nicholas P. 2002. "Mushroom Stem Cells." BioEssays: News and Reviews in Molecular, Cellular and Developmental Biology 24 (10): 949-52. https://doi.org/10.1002/bies.10160.

Montgomery, Sean A., Yasuhiro Tanizawa, Bence Galik, Nan Wang, Tasuku Ito, Takako Mochizuki, Svetlana Akimcheva, et al. 2020. "Chromatin Organization in Early Land Plants Reveals an Ancestral Association between H3K27me3, Transposons, and Constitutive Heterochromatin." Current Biology: CB 30 (4): 573-588.e7. https://doi.org/10.1016/j.cub.2019.12.015.

Nakayama, J., J. C. Rice, B. D. Strahl, C. D. Allis, and S. I. Grewal. 2001. "Role of Histone H3 Lysine 9 Methylation in Epigenetic Control of Heterochromatin Assembly." Science (New York, N.Y.) 292 (5514): 110-13. https://doi.org/10.1126/science.1060118.

Nguyen, Tinh-Suong, Hervé Lalucque, Fabienne Malagnac, and Philippe Silar. 2017. "Prions and Prion-Like Phenomena in Epigenetic Inheritance." In Handbook of Epigenetics (Second Edition), edited by Trygve O. Tollefsbol, 61-72. Academic Press. https://doi.org/10.1016/B978-0-12-8053881.00005-5.

O'Carroll, D., S. Erhardt, M. Pagani, S. C. Barton, M. A. Surani, and T. Jenuwein. 2001a. "The Polycomb-Group Gene Ezh2 Is Required for Early Mouse Development." Molecular and Cellular Biology 21 (13): 4330-36. https://doi.org/10.1128/MCB.21.13.4330-4336.2001.

Ohm, Joyce E., Kelly M. McGarvey, Xiaobing Yu, Linzhao Cheng, Kornel E. Schuebel, Leslie Cope, Helai P. Mohammad, et al. 2007. "A Stem Cell-like Chromatin Pattern May Predispose Tumor Suppressor Genes to DNA Hypermethylation and Heritable Silencing." Nature Genetics 39 (2): 23742. https://doi.org/10.1038/ng1972.

Paoletti, Mathieu, and Sven J. Saupe. 2009. "Fungal Incompatibility: Evolutionary Origin in Pathogen Defense?" BioEssays: News and Reviews in Molecular, Cellular and Developmental Biology 31 (11): 1201-10. https://doi.org/10.1002/bies.200900085.

Paoletti, Mathieu, Sven J Saupe, and Corinne Clavé. 2007. "Genesis of a Fungal Non-Self Recognition Repertoire." PloS One 2 (3): e283. https://doi.org/10.1371/journal.pone.0000283.

Pasini, Diego, Adrian P. Bracken, Michael R. Jensen, Eros Lazzerini Denchi, and Kristian Helin. 2004. "Suz12 Is Essential for Mouse Development and for EZH2 Histone Methyltransferase Activity." The EMBO Journal 23 (20): 4061-71. https://doi.org/10.1038/sj.emboj.7600402.

Pengelly, Ana Raquel, Reinhard Kalb, Katja Finkl, and Jürg Müller. 2015. "Transcriptional Repression by PRC1 in the Absence of H2A Monoubiquitylation." Genes \& Development 29 (14): 1487-92. https://doi.org/10.1101/gad.265439.115. 
Pfaffl, Michael W., Graham W. Horgan, and Leo Dempfle. 2002. "Relative Expression Software Tool (REST) for Group-Wise Comparison and Statistical Analysis of Relative Expression Results in RealTime PCR." Nucleic Acids Research 30 (9): e36. https://doi.org/10.1093/nar/30.9.e36.

Prel, Jean-Baptist du, Gerhard Hommel, Bernd Röhrig, and Maria Blettner. 2009. "Confidence Interval or P-Value?: Part 4 of a Series on Evaluation of Scientific Publications." Deutsches Arzteblatt International 106 (19): 335-39. https://doi.org/10.3238/arztebl.2009.0335.

Rairdan, Gregory J., and Peter Moffett. 2006. "Distinct Domains in the ARC Region of the Potato Resistance Protein Rx Mediate LRR Binding and Inhibition of Activation." The Plant Cell 18 (8): 208293. https://doi.org/10.1105/tpc.106.042747.

Ramírez, Fidel, Devon P. Ryan, Björn Grüning, Vivek Bhardwaj, Fabian Kilpert, Andreas S. Richter, Steffen Heyne, Friederike Dündar, and Thomas Manke. 2016. "DeepTools2: A next Generation Web Server for Deep-Sequencing Data Analysis." Nucleic Acids Research 44 (W1): W160-165. https://doi.org/10.1093/nar/gkw257.

Rea, S., F. Eisenhaber, D. O’Carroll, B. D. Strahl, Z. W. Sun, M. Schmid, S. Opravil, et al. 2000. "Regulation of Chromatin Structure by Site-Specific Histone H3 Methyltransferases." Nature 406 (6796): 593-99. https://doi.org/10.1038/35020506.

Reyes-Dominguez, Yazmid, Jin Woo Bok, Harald Berger, E. Keats Shwab, Asjad Basheer, Andreas Gallmetzer, Claudio Scazzocchio, Nancy Keller, and Joseph Strauss. 2010. "Heterochromatic Marks Are Associated with the Repression of Secondary Metabolism Clusters in Aspergillus nidulans." Molecular Microbiology 76 (6): 1376-86. https://doi.org/10.1111/j.1365-2958.2010.07051.x.

Rice, Judd C., Scott D. Briggs, Beatrix Ueberheide, Cynthia M. Barber, Jeffrey Shabanowitz, Donald F. Hunt, Yoichi Shinkai, and C. David Allis. 2003. "Histone Methyltransferases Direct Different Degrees of Methylation to Define Distinct Chromatin Domains." Molecular Cell 12 (6): 1591-98. https://doi.org/10.1016/s1097-2765(03)00479-9.

Ringrose, Leonie, and Renato Paro. 2004. "Epigenetic Regulation of Cellular Memory by the Polycomb and Trithorax Group Proteins." Annual Review of Genetics 38: 413-43. https://doi.org/10.1146/annurev.genet.38.072902.091907.

Rizet, G, and C Engelmann. 1949. "Contribution à l'étude Génétique d'un Ascomycète Tétrasporé: Podospora anserina" 11: 201-304.

Roper, Marcus, Chris Ellison, John W. Taylor, and N. Louise Glass. 2011. "Nuclear and Genome Dynamics in Multinucleate Ascomycete Fungi." Current Biology : CB 21 (18): R786-93. https://doi.org/10.1016/j.cub.2011.06.042.

Saksouk, Nehmé, Elisabeth Simboeck, and Jérôme Déjardin. 2015. "Constitutive Heterochromatin Formation and Transcription in Mammals." Epigenetics \& Chromatin 8: 3. https://doi.org/10.1186/17568935-8-3.

Sanulli, S., M. J. Trnka, V. Dharmarajan, R. W. Tibble, B. D. Pascal, A. L. Burlingame, P. R. Griffin, J. D. Gross, and G. J. Narlikar. 2019. "HP1 Reshapes Nucleosome Core to Promote Phase Separation of Heterochromatin.” Nature 575 (7782): 390-94. https://doi.org/10.1038/s41586-019-1669-2.

Schlesinger, Yeshayahu, Ravid Straussman, Ilana Keshet, Shlomit Farkash, Merav Hecht, Joseph Zimmerman, Eran Eden, et al. 2007. "Polycomb-Mediated Methylation on Lys27 of Histone H3 PreMarks Genes for de Novo Methylation in Cancer." Nature Genetics 39 (2): 232-36. https://doi.org/10.1038/ng1950.

Schotanus, Klaas, Jessica L. Soyer, Lanelle R. Connolly, Jonathan Grandaubert, Petra Happel, Kristina M. Smith, Michael Freitag, and Eva H. Stukenbrock. 2015. "Histone Modifications Rather than the Novel Regional Centromeres of Zymoseptoria tritici Distinguish Core and Accessory Chromosomes." Epigenetics \& Chromatin 8: 41. https://doi.org/10.1186/s13072-015-0033-5.

Schuettengruber, Bernd, and Giacomo Cavalli. 2009. "Recruitment of Polycomb Group Complexes and Their Role in the Dynamic Regulation of Cell Fate Choice." Development (Cambridge, England) 136 (21): 3531-42. https://doi.org/10.1242/dev.033902.

Silar, P., J. M. Dauget, V. Gautier, P. Grognet, M. Chablat, S. Hermann-Le Denmat, A. Couloux, P. Wincker, and R. Debuchy. 2019. "A Gene Graveyard in the Genome of the Fungus Podospora comata." Mol Genet Genomics 294 (1): 177-90. https://doi.org/10.1007/s00438-018-1497-3.

Silar, P, and M Picard. 1994. "Increased Longevity of EF-1 Alpha High-Fidelity Mutants in Podospora 
anserina." Journal of Molecular Biology 235 (1): 231-36. doi: 10.1016/s0022-2836(05)80029-4.

Silar, Philippe. 2013. "Podospora anserina: From Laboratory to Biotechnology." In Genomics of Soiland Plant-Associated Fungi, edited by Benjamin A. Horwitz, Prasun K. Mukherjee, Mala Mukherjee, and Christian P. Kubicek, 283-309. Berlin, Heidelberg: Springer Berlin Heidelberg.

https://doi.org/10.1007/978-3-642-39339-6_12.

Simon, Jeffrey A., and Robert E. Kingston. 2013. "Occupying Chromatin: Polycomb Mechanisms for Getting to Genomic Targets, Stopping Transcriptional Traffic, and Staying Put." Molecular Cell 49 (5): 808-24. https://doi.org/10.1016/j.molcel.2013.02.013.

Singh, P. B., J. R. Miller, J. Pearce, R. Kothary, R. D. Burton, R. Paro, T. C. James, and S. J. Gaunt. 1991. "A Sequence Motif Found in a Drosophila Heterochromatin Protein Is Conserved in Animals and Plants." Nucleic Acids Research 19 (4): 789-94. https://doi.org/10.1093/nar/19.4.789.

Strahl, B. D., and C. D. Allis. 2000. "The Language of Covalent Histone Modifications." Nature 403 (6765): 41-45. https://doi.org/10.1038/47412.

Strom, Amy R., Alexander V. Emelyanov, Mustafa Mir, Dmitry V. Fyodorov, Xavier Darzacq, and Gary H. Karpen. 2017. "Phase Separation Drives Heterochromatin Domain Formation." Nature 547 (7662): 241-45. https://doi.org/10.1038/nature22989.

Studt, Lena, Slavica Janevska, Birgit Arndt, Stefan Boedi, Michael Sulyok, Hans-Ulrich Humpf, Bettina Tudzynski, and Joseph Strauss. 2016. "Lack of the COMPASS Component Ccl1 Reduces H3K4 Trimethylation Levels and Affects Transcription of Secondary Metabolite Genes in Two PlantPathogenic Fusarium Species." Frontiers in Microbiology 7: 2144. https://doi.org/10.3389/fmicb.2016.02144.

Studt, Lena, Sarah M. Rösler, Immo Burkhardt, Birgit Arndt, Michael Freitag, Hans-Ulrich Humpf, Jeroen S. Dickschat, and Bettina Tudzynski. 2016. "Knock-down of the Methyltransferase Kmt6 Relieves H3K27me3 and Results in Induction of Cryptic and Otherwise Silent Secondary Metabolite Gene Clusters in Fusarium fujikuroi." Environmental Microbiology 18 (11): 4037-54. https://doi.org/10.1111/1462-2920.13427.

Tamaru, H., and E. U. Selker. 2001. "A Histone H3 Methyltransferase Controls DNA Methylation in Neurospora crassa." Nature 414 (6861): 277-83. https://doi.org/10.1038/35104508.

Thalheim, Torsten, Maria Herberg, Markus Loeffler, and Joerg Galle. 2017. "The Regulatory Capacity of Bivalent Genes-A Theoretical Approach." International Journal of Molecular Sciences 18 (5). https://doi.org/10.3390/ijms18051069.

Trojer, Patrick, and Danny Reinberg. 2007. "Facultative Heterochromatin: Is There a Distinctive Molecular Signature?" Molecular Cell 28 (1): 1-13. https://doi.org/10.1016/j.molcel.2007.09.011.

Vandesompele, Jo, Katleen De Preter, Filip Pattyn, Bruce Poppe, Nadine Van Roy, Anne De Paepe, and Frank Speleman. 2002. "Accurate Normalization of Real-Time Quantitative RT-PCR Data by Geometric Averaging of Multiple Internal Control Genes." Genome Biology 3 (7). https://doi.org/10.1186/gb-2002-3-7-research0034.

Veluchamy, Alaguraj, Achal Rastogi, Xin Lin, Bérangère Lombard, Omer Murik, Yann Thomas, Florent Dingli, et al. 2015. "An Integrative Analysis of Post-Translational Histone Modifications in the Marine Diatom Phaeodactylum tricornutum." Genome Biology 16 (May): 102. https://doi.org/10.1186/s13059015-0671-8.

Voigt, Philipp, Gary LeRoy, William J. Drury, Barry M. Zee, Jinsook Son, David B. Beck, Nicolas L. Young, Benjamin A. Garcia, and Danny Reinberg. 2012. "Asymmetrically Modified Nucleosomes." Cell 151 (1): 181-93. https://doi.org/10.1016/j.cell.2012.09.002.

Wang, J., J. Mager, Y. Chen, E. Schneider, J. C. Cross, A. Nagy, and T. Magnuson. 2001. "Imprinted X Inactivation Maintained by a Mouse Polycomb Group Gene." Nature Genetics 28 (4): 371-75. https://doi.org/10.1038/ng574.

Wickham, Hadley. 2009. Ggplot2: Elegant Graphics for Data Analysis. Use R! New York: SpringerVerlag. https://doi.org/10.1007/978-0-387-98141-3.

Widschwendter, Martin, Heidi Fiegl, Daniel Egle, Elisabeth Mueller-Holzner, Gilbert Spizzo, Christian Marth, Daniel J. Weisenberger, et al. 2007. "Epigenetic Stem Cell Signature in Cancer." Nature Genetics 39 (2): 157-58. https://doi.org/10.1038/ng1941.

Wiles, Elizabeth T., and Eric U. Selker. 2017. "H3K27 Methylation: A Promiscuous Repressive 
bioRxiv preprint doi: https://doi.org/10.1101/2020.08.21.261065; this version posted August 21, 2020. The copyright holder for this preprint (which was not certified by peer review) is the author/funder. All rights reserved. No reuse allowed without permission.

Chromatin Mark." Current Opinion in Genetics \& Development 43 (April): 31-37. https://doi.org/10.1016/j.gde.2016.11.001. 


\section{Figure legend}

Figure 1. Structure and functions of the histone methyltransferases PaKmt1 and PaKmt6. A. Domain structure of histone methyltransferases PaKmt1 and PaKmt6. Sizes in amino acid (aa) are given (right). Pre-SET (red, IPR007728), SET (orange, IPR001214) and Post-SET (green, IPR003616) conserved domains are required for H3K9 methyltransferase activity of KMT1 homologue proteins. CXC (yellow, IPR026489) is a cysteine rich conserved domain located in the H3K27 methyltransferase catalytic domain of KMT6 homologues. The H3K9me3 reader PaHP1 is also present in $P$. anserina's genome. It displays canonic chromo domain (PS00598) and chromo shadow domain (PS50013). B. Detection of histone post-translational modifications Western-immunoblot analysis of H3K9me3, H3K27me3 and H3K4me3 modifications in wild-type (WT); single mutants $\triangle$ PaKmt1, $\triangle$ PaKmt6, $\triangle$ PaSir2 (Boivin, Gaumer, and Sainsard-Chanet 2008), $\triangle P a H P 1$, $\triangle P a R i d$ (Grognet et al. 2019); double mutant $\triangle P a K m t 1 \triangle P a K m t 6$; complemented strains

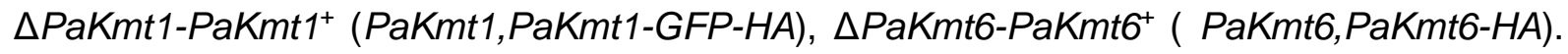
Nuclear protein samples were extracted from protoplasts. A total of $35 \mu \mathrm{g}$ of nuclear protein was loaded in each lane. Antibodies were raised against native $\mathrm{H} 3$ histone $(\mathrm{H} 3), \mathrm{H} 3 \mathrm{~K} 9 \mathrm{me}$, H3K27me3 and H3K4me3 modifications. Expected size of histone 3 is $17 \mathrm{kDa}$ (arrows). Probing with anti-H3K27me3 and anti-H3 antibodies reveals an additional signal, lower than 15 kDa. Deletion of either PaSir2 or PaRid, encoding respectively a NAD+-dependent histone deacetylase and a putative DNA-methyltransferase, has no impact on methylation of H3K9me3, H3K27me3 or H3K4me3.

Figure 2. Distribution of histone marks in the wild-type strain. A. Wild-type distribution of H3K4me3, H3K9me3 and H3K27me3 on chromosomes one and five. Patterns are represented with the same scale on chromosomes one (top) and five (bottom) of $P$. anserina. H3K4me3 (green), H3K9me3 (red), H3K27me3 (blue). Both centromeres are depicted (pink). The "Mat region" (orange) correspond to the $800 \mathrm{kpb}$ region devoid of recombination and containing the mating-type locus (Grognet, Bidard, et al. 2014). Repeated sequences are depicted in light blue. B. Visualization of histone marks localization across the genome. 
Each of the seven $P$. anserina's chromosomes is depicted three times so each of the three marks can be mapped even if overlapping. Presence of H3K4me3, H3K9me3 and H3K27me3 is shown in green, red and blue, respectively. Centromeres are depicted as a neck on the chromosomes. The grey telomeric area of chromosome 3 represents the rDNA array. A close-up of the small arm of chromosome 7 is displayed to show the precise localization of the marks that cannot be seen on the somewhat rougher whole genome visualization.

Figure 3. Relations of histone marks and genes in the wild-type strain. A. Peak sizes distribution in the wild-type strain. Box plots showing sizes (in base pair) of the MACS2 predicted peak of H3K4me3, H3K9me3 and H3K27me3 are shown in green, red and blue, respectively. The box is delimited, bottom to top, by the first quartile, the median and the third quartile. Numbers on the right of each box represents the number of peaks detected. Outliers are not shown. B. Heatmaps of histone marks on different genomic regions in the wildtype strain. Heatmaps and plots of normalized H3K4me3, H3K9me3 and H3K27me3 ChIP signal in wild type strain over promoters (left panel), CDS (middle) and repeats (right panel) regions. H3K4me3 clusters 2 and 3 on promoter regions are actually identical. The difference is due to the fact that genomic regions coordinates are provided regardless of their orientation. Hence, the two clusters are composed of active promoter regions in both orientation and shows that $\mathrm{H} 3 \mathrm{~K} 4 \mathrm{me} 3$ is enriched close to the start codon. The same thing can be seen in a lesser extent on the middle panel. C. Violin plots of gene expression according to the histone mark. Gene expression was inferred from the TPM (Transcripts Per Kilobase Million) values calculated in (P. Silar et al. 2019). Log2 of the TPM values were plotted according to the marks detected of the CDS. The three lines of the violin plot represent the first quartile, the median and the third quartile. The white dot and the number above represent the mean. The numbers on top represent the number of gene in each category. D. Euler diagram showing overlap between the different histone marks. H3K4me3, H3K9me3 and H3K27me3 are represented by the green, red and blue areas, 
respectively. The numbers indicate the number of base pair covered by each mark. Overlap between areas shows the number of base pair covered by two marks.

Figure 4. Distribution of histone marks in $\triangle P a K m t 6$ and $\triangle P a K m t 1$ strains. A. Visualization of $\mathrm{H} 3 \mathrm{~K} 9 \mathrm{me} 3$ and H3K27me3 localization across the genome in wild-type and mutant backgrounds. Each of the seven $P$. anserina's chromosomes is depicted three times corresponding to (a) the wild type strain, (b) the $\Delta P a K m t 1$ strain and (c) the $\Delta P a K m t 6$ strain. Centromeres are depicted as a neck on the chromosomes. The grey telomeric area of chromosome 3 represents the rDNA. A close-up of the small arm of chromosome 7 is displayed to show the precise localization of the marks that cannot be seen on the somewhat rougher whole genome visualization. B. Heatmaps of H3K4me3 and H3K27me3 on CDS and repeats in the $\Delta P a K m t 1$ and $\Delta$ PaKmt6 mutant strains. Heatmaps and plots of normalized H3K4me3 and H3K27me3 ChIP signal in mutant and wild type strains over CDS regions. C. Peak sizes distribution in the $\Delta P a K m t 1$ and $\Delta P a K m t 6$ mutant strains. Box plots showing sizes in base pair of the MACS2 predicted peak of H3K4me3, H3K9me3 and H3K27me3 are shown in green, red and blue, respectively. The box is delimited, bottom to top, by the first quartile, the median and the third quartile. Numbers on top of each box represents the number of peaks detected. Outliers are not shown.

Figure 5. Gene expression in the $\Delta P a K m t 6$ strain and growth features of the $\Delta P a K m t 6$ and $\Delta$ PaKmt1 strains. A. Selected genes relative expression in $\Delta P a K m t 6$ mutant strains. Upper panel: histone marks in the wild-type strain. For each selected gene, a black dot shows the presence of the mark. Conversely a white dot shows its absence. Pa_4_1170 and Pa_1_16300 encode two putative Glycoside Hydrolases from family 7 and 61, respectively (Espagne et al. 2008). Pa_6_7270 and Pa_6_7370 belong to a secondary metabolite gene cluster located on one of chromosome 7 sub-telomeric regions. Pa_1_1880 encodes the putative DNA repair RAD50 protein. Pa_7_9210 encodes a putative exonuclease. They are both readily expressed at all stages of the life cycle and their expression remained unchanged in any of the two mutant backgrounds. Pa_5_10 encodes the meiotic drive element Spok2 located on Chromosome 5 (Grognet, Lalucque, et al. 
2014b). Middle panel: normalized expression ratio of the selected genes relative to the wildtype strain. Overexpression (Fold change $\geq 2$, dark black line): Pa_1_6263: expression ratio $=25,987, \mathrm{p}$-value $=0.001 ;$ Pa_4_1170: expression ratio $=6,375$, p-value $=0.004 ;$ Pa_1_16300: expression ratio $=7,588, \mathrm{p}$-value $=0.003 ;$ Pa_6_7270: expression ratio $=$ 5,280, p-value < $0001 ; P a \_6 \_7370$ : expression ratio $=6,016, \mathrm{p}$-value $=0.002 ;$ Pa_1_1880: expression ratio $=1.216, p$-value $=0.192 ; \mathrm{Pa} \_7 \_9210:$ expression ratio $=0.756, p$-value $=$ 0.061; Pa_5_10: expression ratio $=2,212, \mathrm{p}$-value $<0.001$. Three normalization genes (AS1, GPD and PDF2) were selected with geNorm (Vandesompele et al. 2002) among eight housekeeping genes. Average expression stability of these three normalization genes is $M=$ 0.230 and their pairwise variation is $\mathrm{V} 3 / 4=0.071$. See Material and Methods for details and Table S3 for Cq and selection of normalization genes. Lower panel: histone marks in the $\Delta$ PaKmt6 mutant strain. They are indicated as in the upper panel (see above). B. Phenotypic characterization of $\Delta P a K m t 1$ and $\Delta P a K m t 6$ single mutant strains as well as $\Delta$ PaKmt1DPaKmt6 double mutant strains. The mat+ strains of the indicated genotypes were grown on $\mathrm{M} 2$ minimal medium for 5 days at $27^{\circ} \mathrm{C}$ (right) or $\mathrm{M} 2$ plus yeast extract for 3 days at $27^{\circ} \mathrm{C}$ (left). C. Vegetative growth kinetics of $\Delta$ PaKmt1 and $\Delta$ PaKmt6 single mutant strains on M2 minimum medium. See Material and Methods section for details.

Figure 6. Sexual features of the $\Delta P a K m t 6$ strain. A. Spermatium production in wildtype and mutant backgrounds. See Material and Methods section for details. B. $\Delta$ PaKmt6 homozygote crosses result in non-homogeneous distribution of late appearing fruiting bodies showing multiple morphological defects. These crippled structures that could either be due to either lack of neck, lack of ostiols, lack setae, or non-canonical pear-shape envelopes, supernumerary necks, misdirected orientation, upside down growth in agar, fusions of fruiting bodies, etc.

Figure 7. Histone marks in the $\triangle P a H P 1$ strain. A. Visualization of histone marks localization across the genome in the $\triangle P a H P 1$ strain. Each of the seven $P$. anserina's chromosomes is depicted three times so each of the three marks can be mapped even if 
overlapping. Presence of H3K4, H3K9 and H3K27 trimethylation is shown in green, red and blue, respectively. Centromeres are depicted as a neck on the chromosomes. The grey telomeric area of chromosome 3 represents the rDNA. A close-up of the small arm of chromosome 7 is displayed to show the precise localization of the marks that cannot be seen on the somewhat rougher whole genome visualization. B. Heatmaps of H3K4me3, H3K9me3 and H3K27me3 on CDS and repeats in the $\triangle P a H P 1$ mutant strain.

Heatmaps and plots of normalized H3K4me3, H3K9me3 and H3K27me3 ChIP signal in $\triangle P a H P 1$ mutant and wild type strains over CDS regions (left panel) and repeat region (right panel). C. Peak sizes distribution in the $\triangle P a H P 1$ mutant strain. Box plots showing sizes in base pair of the MACS2-predicted peak of H3K4, H3K9 and H3K27 trimethylation are shown in green, red and blue, respectively. The box is delimited, bottom to top, by the first quartile, the median and the third quartile. Numbers on top of each box represents the number of peaks detected. Outliers are not shown.

\section{Figure 8. Subcellular localization of PaHP1}

Fluorescence microscopy pictures show PaHP1-GFP chimeric protein during vegetative growth in mycelium. Nuclei are marked using the PaH1-mCherry allele that encodes histone $\mathrm{H} 1$ protein tagged with mCherry fluorophore (red) in the indicated strain (left) or with DAPI $(1 \mu \mathrm{g} / \mu \mathrm{l}$, blue) for $\triangle P a K m t 6, P a H P 1-G F P$ strain. Indicated scale is the same for all the pictures $(2 \mu \mathrm{m})$. PaHP1-GFP displays nuclear localization and forms foci that likely correspond to heterochromatin domains (arrows). This specific localization is disturbed only in $\triangle P a K m t 1$ mutants.

Table 1: Mycelium growth at different temperatures. Relative growth rates (WT \%) at optimal temperature $\left(27^{\circ} \mathrm{C}\right)$ and sub-optimal temperature $\left(11^{\circ} \mathrm{C}\right.$ and $\left.35^{\circ} \mathrm{C}\right)$ on $\mathrm{M} 2$ minimal medium. $\triangle P a K m t 6$ mutant strains display irregular and slow growth at $27^{\circ} \mathrm{C}$ and $35^{\circ} \mathrm{C}$, which cannot be quantified. At $11^{\circ} \mathrm{C}$, growth is too weak to be assayed (ND). At $27^{\circ} \mathrm{C}$, double and triple mutants containing the $\triangle P a K m t 6$ allele display aggravated $\triangle P a K m t 6$-like growth defects. 
Table 2: Ascospore germination efficiency. Number of germinated ascospores out of a 100 issued from homozygote $\triangle P a K m t 6$ crosses and transferred on appropriate germination medium. WT means wild-type germination efficiency (>95\%).

\section{Table 3: Phenotypes associated to either loss of KMT1, KMT6 or HP1 in fungal} species. Growth \& development : macroscopic major defects caused by the specified mutant allele. Chromatin \& regulation : H3K9me3 or H3K27me3 modification status into the mutant background and major impact on regulation of gene expression. Technics used to characterize chromatin status are listed. ChiP-seq : chromatin immunoprecipitation followed by sequencing, Hi-C: genome-wide chromosome conformation capture followed by sequencing, WB: Western-blot. 


\section{Supplementary Figure 1. Conserved structure and phylogenetic analysis of histone} methyltransferases involved in heterochromatin assembly Domain structure comparison of histone methyltransferase $\mathrm{Kmt1}$ (A) and Kmt6 (B). Sizes in amino acid (aa) are given (right). Pre-SET (red, IPR007728), SET (orange, IPR001214) and Post-SET (green, IPR003616) conserved domains are required for H3K9 methyltransferase activity of KMT1 homologue proteins. SRA (SET and RING finger-associated, purple, IPR003105) and SANT (blue, IPR001005) are protein-protein interaction domains. CXC (yellow, IPR026489) is a cysteine rich conserved domain located in the H3K27 methyltransferase catalytic domain of Kmt6 homologues. Filamentous fungi: Podospora anserina (Pa), Neurospora Crassa (Nc), Fusarium graminearum (Fg), Epichloë festucae (Ef), Aspergillus nidulans (An); yeasts: Schizosaccharomyces pombe (Sp) and Cryptococcus neoformans (Cn); the worm Caenorhabditis elegans (Ce), the fruit-fly Drosophila melanogaster (Dm), the mouse Mus musculus (Mm) and the model plant Arabidopsis thaliana (At). Accession numbers for proteins used in alignments are listed in Table S5. C. Phylogenetic analysis of Kmt1 and Kmt6 histone methyltransferase homologs. This tree regroups both families of H3K9 (blue) and H3K27 (red) methyltransferase proteins from fungi, plant and metazoan. Both $P$. anserina's histone methyltransferases are marked with rectangles. They cluster two different clades of histone methyltransferase. Their evolutionary history is canonical since the topology of the two branches of the tree is consistent with the evolution of species. Bootstraps are given. Filamentous fungi: Podospora anserina (Pa), Neurospora crassa (Nc), Fusarium graminearum (Fg), Trichoderma reesei (Tr), Epichloë festucae (Ef) Botrytis cinerea (Bc), Magnaporthe oryzae (Mo), Zymoseptoria tritici (Zt), Leptosphaeria maculans (Lm), Aspergillus nidulans (An), Aspergillus fumigatus (Af), Penicillium oxalicum (Po), Ascobolus immersus (Ai), Puccinia graminis (Pg), Pneumocystis jirovecii (Pj), Conidiobolus coronatus (Cc), Mucor circinelloides (Mc); yeasts: Schizosaccharomyces pombe (Sp) and Cryptococcus neoformans (Cn); the worm Caenorhabditis elegans (Ce), the fruit-fly Drosophila melanogaster $(\mathrm{Dm})$, the mouse Mus musculus $(\mathrm{Mm})$ and the model plant Arabidopsis thaliana (At). Accession numbers for proteins used in alignments are listed in Table S5. 


\section{Supplementary Figure 2. Expression kinetics PaKmt1 in wild-type strain}

Upper panel: RT-PCR on RNA extraction from wild-type vegetative growing mycelium (one day and four days), perithecia (two days and four days after the fertilization), and input genomic DNA. PaKmt1 CDS was predicted to be made of two exons separated by a $62 \mathrm{bp}$ intron (positions 49-110). However, two amplicons of distinct sizes are obtained, corresponding to both spliced (1108 bp) and unspliced (1170 bp) PaKmt1 mRNAs. MW: molecular weight. Lower panel: schematic representation of the PaKmt1 locus (DNA). mRNA1 corresponds to the spliced form of the transcripts while mRNA2 (1108 bp) corresponds to the unspliced form (1170 bp). Translation of the unspliced form would lead to a premature termination and thus to a truncated protein. Primers used for the reversetranscription polymerase chain reaction (RT-PCR) are drawn as arrows above and below the PaKmt1 CDS.

\section{Supplementary Figure 3. Epigenetic landscapes in wild-type and heterochromatin} mutant strains of $\boldsymbol{P}$. anserina. Panorama of genome-wide peak localization for each genotype, wild-type, $\triangle P a K m t 1, \triangle P a K m t 6$ and $\triangle P a H P 1$ strains. Telomeres sequences were arbitrarily defined as the segment going from the end of each arm of the chromosomes to the first annotated gene (at the exception of the rDNA cluster localized on chromosome 3) and centromeres are indicated. Mat region = Non recombining region containing the mating-type locus as defined in (Grognet, Bidard, et al. 2014).

Supplementary Figure 4. H3K27me3, H3K4me3 and H3K9me3 proportions on the $P$. anserina's chromosomes in the WT, $\triangle P a K m t 1, \triangle P a K m t 6$ and $\triangle P a H P 1$ mutant strains. Plot showing the percentage of each chromosome covered with H3K4me3 (green), H3K9me3 (red) and H3K27me3 (blue). The coverage is the sum of all MACS2 predicted peak size.

\section{Supplementary Figure 5. Molecular characterization of knockout mutants by Southern}

blot hybridization. Schematic representations of the endogenous and disrupted loci are given (Left). Replacement by homologous recombination of the wild type PaKmt1 allele by the disrupted $\triangle$ PaKmt1 allele results in the substitution of a $2.4 \mathrm{~kb}$ and $5.7 \mathrm{~kb}$ EcoRV 
fragments by a unique $11 \mathrm{~kb} P s t l$ fragment as revealed by hybridization of the $5^{\prime}$ and $3^{\prime}$ digoxygenin-labeled probes (dashed rectangles PaKmt1 locus). Replacement by homologous recombination of the wild type PaKmt6 allele by the disrupted $\Delta P a K m+6$ allele results in the substitution of a unique $6.5 \mathrm{~kb} K p n l$ fragment by two 1.8 and $2.4 \mathrm{~kb} K p n l$ fragments as revealed by hybridization of the 5' and 3' digoxygenin-labeled probes (dashed rectangles PaKmt6 locus). A second verification has been made with the Hindll enzymes and the same probes shows the substitution of two $1.9 \mathrm{~kb}$ and $4.6 \mathrm{~kb}$ Hindlll fragments by a 3.7 kb Hindll fragment as revealed by hybridization of the 5' and 3' digoxygenin-labeled probes.

Supplementary Figure 6. Antibody specificity analysis. Dot blot experiment results using H3K9me3 and H3K27me3 peptides (left) at different concentration (top). Membranes were inoculated with the corresponding antibody (right). Signal intensity comparison shows a cross reactivity between anti-H3K9me3 antibody and $\mathrm{H} 3 \mathrm{~K} 27 \mathrm{me} 3$ evaluated at $3 \%$ compared to the immunogen reaction.

\section{Supplementary Figure 7. Relative expression of selected genes in the $\Delta$ PaKmt1} mutants. Caption as in Figure 5A. No significant fold-change was assayed, except for Pa_1_6263 and Pa_6_7370, which both lost the H3K27me3 mark and were upregulated and downregulated, respectively. $P a \_1 \_6263$ : expression ratio $=1.855, \mathrm{p}$-value $=0.002$; Pa_4_1170: expression ratio $=1.535, \mathrm{p}$-value $=0.094 ; P a \_1 \_16300$ : expression ratio $=$ 1.393, $\mathrm{p}$-value $=0.092 ; P a \_6 \_7270:$ expression ratio $=1.397, \mathrm{p}$-value $=0.035 ; P a \_6 \_7370$ : expression ratio $=0.696, p$-value $=0.006 ; \mathrm{Pa} \_1 \_1880$ : expression ratio $=1.422, \mathrm{p}$-value $=$ 0.034; Pa_7_9210 $:$ expression ratio $=1.082, \mathrm{p}$-value $=0.252 ; \mathrm{Pa}$ _5_10: expression ratio $=$ $0.759, \mathrm{p}$-value $=0.017$

\section{Supplementary Figure 8. Growth features of $\Delta P a K m t 1$ strain. A. Experimental} procedure to test growth resuming capabilities of $\Delta$ PaKmt1 strain. To set up this restart test, mycelium implants issued from germination thalli were inoculated onto a fresh M2 medium and incubated at $27^{\circ} \mathrm{C}$ for eight days (step 1). Two independent plugs from each location (purple cross), eight-day stationary phase (plug\#3); pink cross, four-day stationary 
phase (plug\#2); and green cross, growing phase (plug\#1) were then transferred on a fresh M2 solid medium and incubated for three days at $27^{\circ} \mathrm{C}$ (step 2). The growing phase of each thallus originating from step 2 (green margins) were transplanted again on fresh M2 solid medium and incubated at $27^{\circ} \mathrm{C}$ for three days (step 3). Growth restart from stationary phase was impaired for $\triangle P a K m t 1$ mutants, which resulted in smaller and thinner colonies than the wild-type ones (white arrows), whereas continuous growth (plug\#1) was not altered. Complemented $\triangle P a K m t 1-P a K m t 1^{+}$strains behaved as wild-type strains. As control experiments (step 3), we then transferred mycelia from growing margins (marked in green, step 2) of thalli deriving from Plug\#1, Plug\#2 and Plug\#3. In this case, $\Delta$ PaKmt1 mutants did not show any delay to resume growth (orange arrows). B. Crippled growth test for $\Delta$ PaKmt1 strain. Crippled growth (CG) process can be shown using a 'band test'. Strains were incubated on $\mathrm{M} 2$ medium for 7 days at $27^{\circ} \mathrm{C}$. Two 1-mm-width slices of agar were then inoculated onto fresh M2 media with or without yeast extract (YE) in the following method for 3 days. Picture shows actively growing apical hyphae (right) and resting stationary phase hyphae (left). The surface side of the slice on plate has been orientated at the top. CG is a degenerative process caused by $\mathrm{C}$ element production in the stationary phase. When inoculated on yeast extract medium, it displays slow growth, alteration of pigmentation, inability to differentiate aerial hyphae, and female sterility. $\triangle P a M p k 1$ mutant strain is impaired for CG development while PDC2208 strain is supposed to display CG on M2 medium without yeast extract. The single $\triangle P a K m t 1$ mutants are not impaired for $C G$.

Supplementary Figure 9. Longevity tests for $\Delta P a K m t 1$ and $\Delta P a K m t 6$ strains. Graphs show the maximal growth length on $\mathrm{M} 2$ medium at $27^{\circ} \mathrm{C}$ in race tube for wild-type (WT), $\triangle P a K m t 1$ and $\triangle P a K m t 6$ mutant strains for both mating type. Data correspond to the mean of 3 technical samples from 5 biological samples.

Supplementary Figure 10 . Role of PaKmt6 during sexual development. A. Heterozygote oriented crosses between wild-type strains (WT) and $\Delta$ PaKmt6 mutants. Fertilization of wild-type ascogonia with $\Delta P a K m t 6$ male gametes results in normal fruiting body development displaying a single neck (black arrow), while fertilization of $\triangle$ PaKmt6 
ascogonia with wild-type spermatia results in fewer crippled $\Delta P a K m t 6$-like fruiting bodies (two necks are indicated by two black arrows). These features indicate that PaKmt6 is a maternal gene. B. Mosaic analyses using the $\Delta$ mat mutant strain. Wild-type or $\Delta P a K m t 6$ mat+ and mat- strains were mixed with or without $\Delta$ mat mycelium and inoculated onto fresh M2 medium. After a week of incubation on M2 medium, $\triangle P a K m t 6$ dikaryon displayed crippled and mis-orientated perithecia, resulting in scattered and reduced ascospore production. The tricaryon $\triangle P a K m t 6$ mat+ / $\triangle P a K m t 6$ mat- / $\Delta$ mat showed nearly wild-type restoration of mycelium growth, as well as perithecia and ascospore production. These features confirm that PaKmt6 is a gene expressed in the maternal tissues of the perithecium and not in its zygotic tissues (centrum).

Supplementary Figure 11. Structure and evolutionary relationships of HP1 orthologues. A. Domain structure comparison of heterochromatin protein-1 homologues. Sizes in amino acid (aa) are given (right). Chromodomain (turquoise, IPR023780) recognizes H3K9me2/3 histone modification. Chromo-shadow (dark green, IPR008251) is a protein-protein interaction domain. Filamentous fungi: Podospora anserina (Pa), Neurospora crassa (Nc), Fusarium graminearum (Fg), Aspergillus nidulans (An); yeasts: Schizosaccharomyces pombe (Sp); the fruit-fly Drosophila melanogaster (Dm), the mouse Mus musculus (Mm) and the model plant Arabidopsis thaliana (At). Accession numbers for proteins used in alignments are listed in Table S5. B. Tree showing evolution of some HP1 orthologues from fungi, plant and metazoans. The $P$. anserina's heterochromatin protein-1 homologue is marked with a rectangle. Bootstraps are given. Filamentous fungi: Podospora anserina (Pa), Neurospora crassa (Nc), Fusarium graminearum $(\mathrm{Fg})$, Trichoderma reesei (Tr), Epichloë festucae (Ef) Botrytis cinerea (Bc), Magnaporthe oryzae (Mo), Zymoseptoria tritici (Zt), Leptosphaeria maculans (Lm), Aspergillus nidulans (An), Aspergilus fumigatus (Af), Penicillium oxalicum (Po), Ascobolus immersus (Ai), Puccinia graminis (Pg), Pneumocystis jirovecii (Pj), Conidiobolus coronatus (Cc), Mucor circinelloides (Mc); yeasts: Schizosaccharomyces pombe (Sp) and Cryptococcus neoformans (Cn); the worm Caenorhabditis elegans (Ce), the fruit-fly Drosophila 
melanogaster $(\mathrm{Dm})$, the mouse Mus musculus $(\mathrm{Mm})$ and the model plant Arabidopsis thaliana (At). Accession numbers for proteins used in alignments are listed in Table S5.

Supplementary Figure 12. Growth features of the $\triangle P a H P 1$ strain. A. Molecular characterization of knockout $\triangle P a H P 1$ mutants by Southern blot hybridization. Replacement by homologous recombination of the wild type PaHP1 allele by the disrupted $\triangle P a H P 1$ allele results in the substitution of a unique $3.4 \mathrm{~kb} E c o R I / E c o R V$ fragment by two 1.4 and $2.5 \mathrm{~kb}$ EcoRI/EcoRV fragments as revealed by hybridization of the 5'UTR and 3'UTR digoxygenin-labeled probes (dashed rectangles PaHP1 locus). B. Vegetative growth of $\triangle P a H P 1$ single mutants and $\triangle P a K m t 1 \Delta P a H P 1$ double mutants compared to wild-type strains. $\triangle P a K m t 1$ and $\triangle P a H P 1$ single mutants are impaired in aerial mycelium production. This defect was more pronounced for the single $\triangle P a H P 1$ mutants. $\triangle P a K m t 1 \triangle P a H P 1$ double mutants show $\triangle$ PaKmt1 morphologic phenotype. The strains have been grown on $\mathrm{M} 2$ medium 3 days. C. Vegetative growth kinetics of $\triangle P a H P 1$ mutants and $\triangle P a K m t 1$ mutants compared to wild-type strains. See Material and Methods section for details. D. Experimental procedure to test growth resuming capabilities of $\triangle P a H P 1$ and double $\Delta$ PaHP1DPaKmt1 mutants. For description of experimental setting see Supplementary Figure 8A. Growth restart from stationary phase (plug\#2 and plug\#3) was impaired for $\triangle P a H P 1$ and double $\triangle P a H P 1 \triangle P a K m t 1$ mutants, which resulted in smaller and thinner colonies than the wild-type ones (white arrows), whereas continuous growth (plug\#1) was not altered. Complemented $\triangle P a H P 1-P a H P 1^{+}$strains behaved as wild-type strains. As control experiments (step 3), we then transferred mycelia from growing margins (marked in green, step 2) of thalli deriving from Plug\#1, Plug\#2 and Plug\#3. In this case, neither $\triangle$ PaKmt1 mutants nor $\triangle P a H P 1 \triangle P a K m t 1$ mutants showed any delay to resume growth (orange arrows). E. Crippled growth test of $\triangle P a H P 1$ single mutants and $\triangle P a H P 1 \Delta P a K m t 1$ double mutants. For description of experimental setting see Supplementary Figure 8B. The single $\triangle P a H P 1$ and the double $\triangle P a K m t 1 \triangle P a H P 1$ mutants are not impaired for $C G$. 


\section{Supplementary Figure 13. Relative expression of selected genes in the $\triangle P a H P 1$ strain.}

Caption as in Figure 5A. No significant fold-change was assayed, except for Pa_1_6263 and Pa_5_10, which both lost the H3K27me3 mark and were upregulated and downregulated, respectively. Pa_1_6263: expression ratio $=2.463, \mathrm{p}$-value $=0.004 ; \mathrm{Pa} \_4 \_1170$ : expression ratio $=1.911, p$-value $=0.032 ; P a \_1 \_16300$ : expression ratio $=0.931, p$-value $=0.693 ;$ Pa_6_7270: expression ratio $=1.527, p$-value $=0.004 ;$ Pa_6_7370: expression ratio $=$ 0.766, $\mathrm{p}$-value $=0.009 ; \mathrm{Pa} \_1 \_1880:$ expression ratio $=1.313, \mathrm{p}$-value $=0.013 ; \mathrm{Pa} \_7 \_9210$ : expression ratio $=0.951, \mathrm{p}$-value $=0.547 ; \mathrm{Pa}$ _5_10: expression ratio $=0.490, \mathrm{p}$-value $=$ 0.006 .

Supplementary Figure 14: Heat map of Spearman's correlation coefficient comparison: clustering analysis of histone marks in wild-type background (WT) and in mutant backgrounds $\triangle P a K m t 1, \triangle P a K m t 6$ and $\triangle P a H P 1$. Mock = IP performed with GFP antibody in absence of GFP tag in P. anserina's genome (see Material and methods). Raw data are given in Table S7.

Table S1: Main features of significantly enriched peaks. Peak numbers, sizes, and genome coverages of $\mathrm{H} 3 \mathrm{~K} 4 \mathrm{me} 3, \mathrm{H} 3 \mathrm{~K} 9$ and $\mathrm{H} 3 \mathrm{~K} 27 \mathrm{me} 3$ modifications, in wild-type background (WT) and in mutant backgrounds $\triangle P a K m t 1, \triangle P a K m t 6$ and $\triangle P a H P 1$.

Table S2. Distribution of H3K4me3, H3K9me3 and H3K27me3 on the complete set of $P$. anserina's gene in wild-type background (WT) and in $\triangle P a K m t 1, \triangle P a K m t 6$ and $\triangle P a H P 1$ mutants. List of genes where H3K4me3 and H3K27me3 can be found overlapping in the wild-type background. Distribution of H3K4me3, H3K9me3 and H3K27me3 on a sub-set of $P$. anserina's secondary metabolite gene clusters in wild-type background (WT) and in PaKmt1, $\triangle P a K m t 6$ and $\triangle P a H P 1$ mutants.

Table S3. $\mathrm{Cq}$ for RT-qPCR experiments and geNorm analysis of candidate normalization genes.

Table S4. Primers used for PCR experiments. A. Primers used for RT-PCR and allele construction experiments. B. Primers used for RT-qPCR experiments.

Table S5. Accession numbers for proteins used in alignments to build the phylogenic trees. 
Table S6. Heat map of Spearman correlation coefficient comparison between ChIP-seq samples. Spearman's correlation coefficient confirms the reproducibility of the experiments since biological replicates cluster together with high correlation coefficient. Moreover, H3K9me3 and H3K27me3 IP share a same cluster that negatively correlates with the H3K4me3 cluster. Both display no correlation with non-IP input and negative sample (Mock and $\mathrm{H} 3 \mathrm{~K} 27 \mathrm{me} 3$ in the $\triangle P a K m t 6)$, which means that IP results are significant and specific.

Table S7. Raw data from the heatmap of Spearman's correlation coefficient comparison in Fig. S14. 
Fig. $1 \mathrm{~A}$
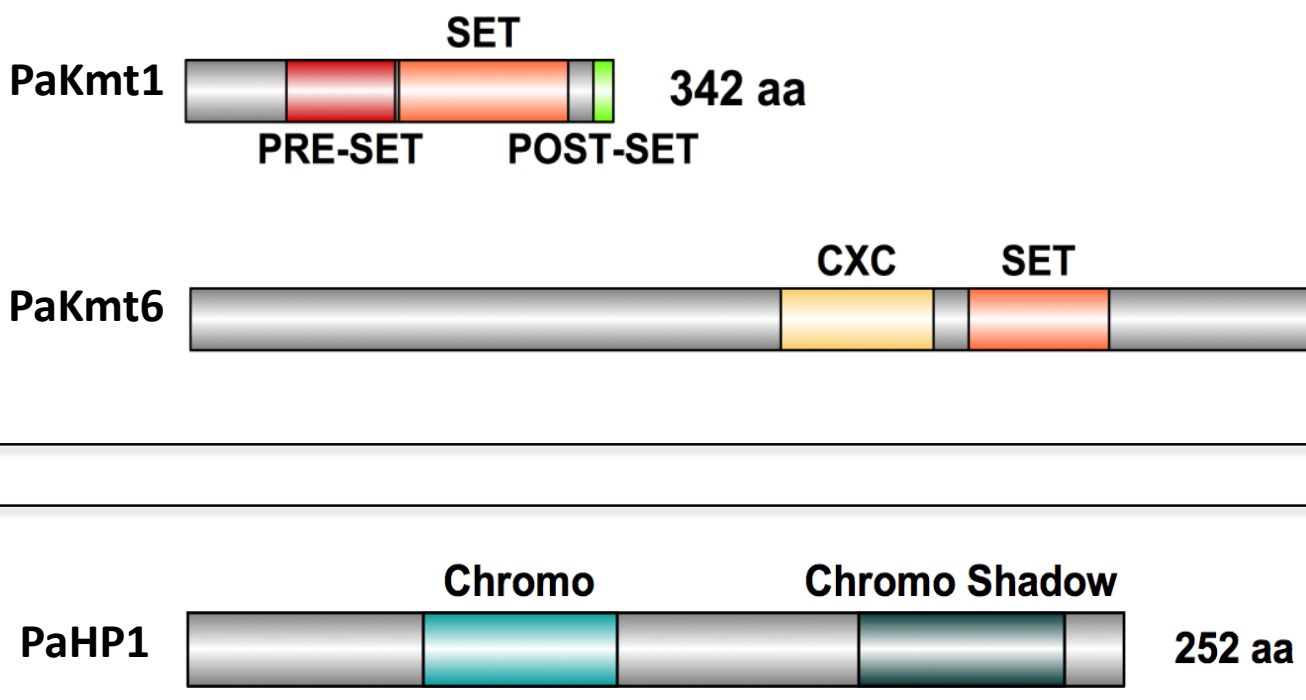

Fig. $1 \mathrm{~B}$

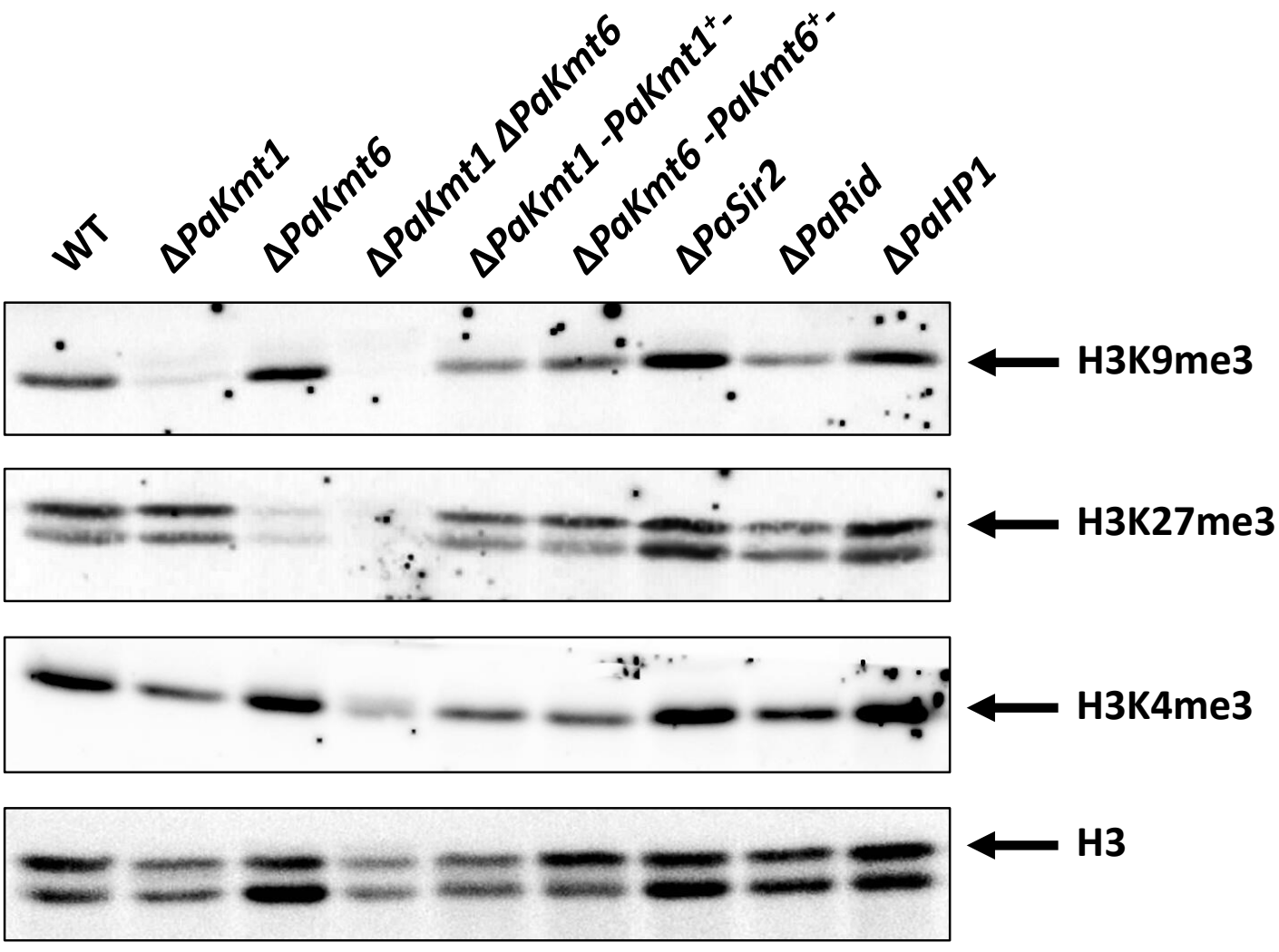


Fig. $2 \mathrm{~A}$

\begin{tabular}{|c|c|c|c|c|c|c|c|c|}
\hline & $1000 \mathrm{bb}$ & $\begin{array}{l}2000 \mathrm{~kb} \\
\perp\end{array}$ & $\begin{array}{c}3000 \mathrm{~kb} \\
\end{array}$ & $\begin{array}{l}4000 \mathrm{~kb} \\
\end{array}$ & $\begin{array}{c}5000 \mathrm{k} \mathrm{h} \\
\end{array}$ & $\begin{array}{c}6000 \mathrm{~kb} \\
\end{array}$ & $7000 \mathrm{~kb}$ & $8000 \mathrm{~kb}$ \\
\hline & & & & & & & 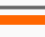 & \\
\hline
\end{tabular}

Chr1

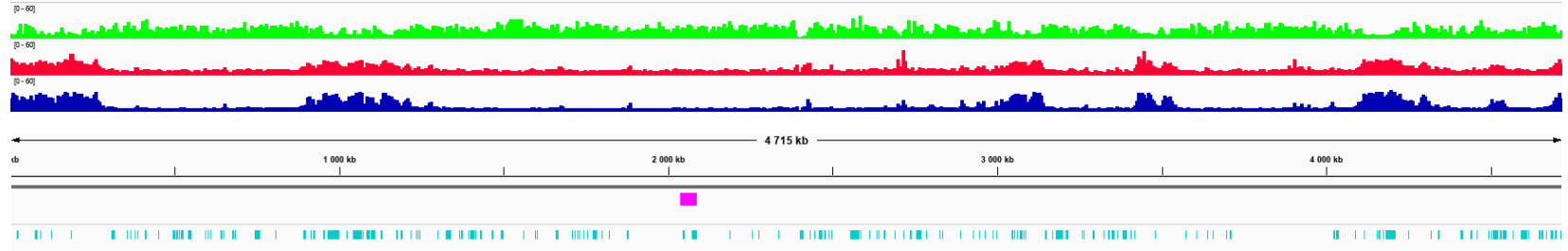

Chr5
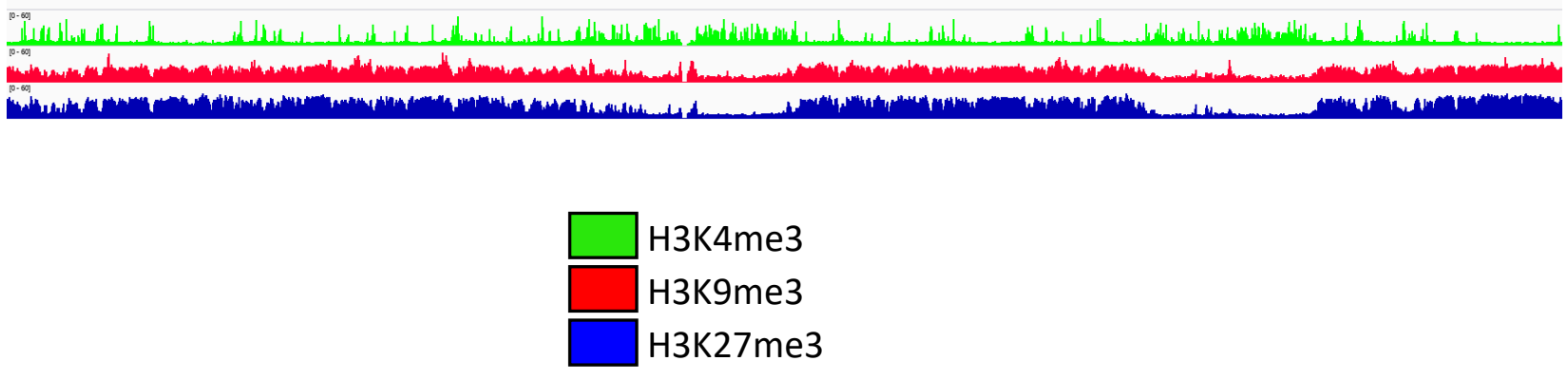

Fig. 2 B Chr1 Chr2 Chr3 Chr4 Chr5 Chr6 Chr7

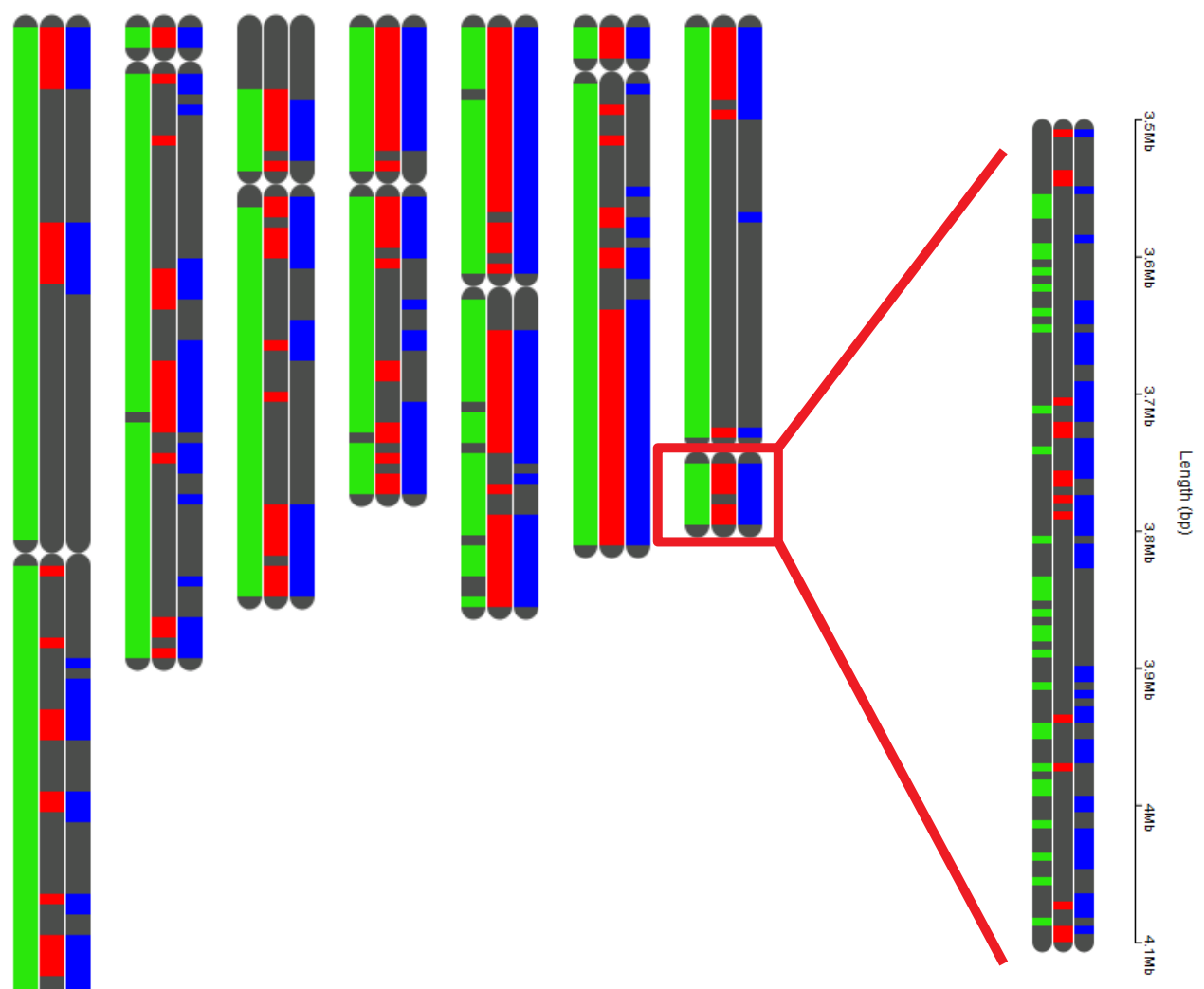


Fig. $3 \mathrm{~A}$

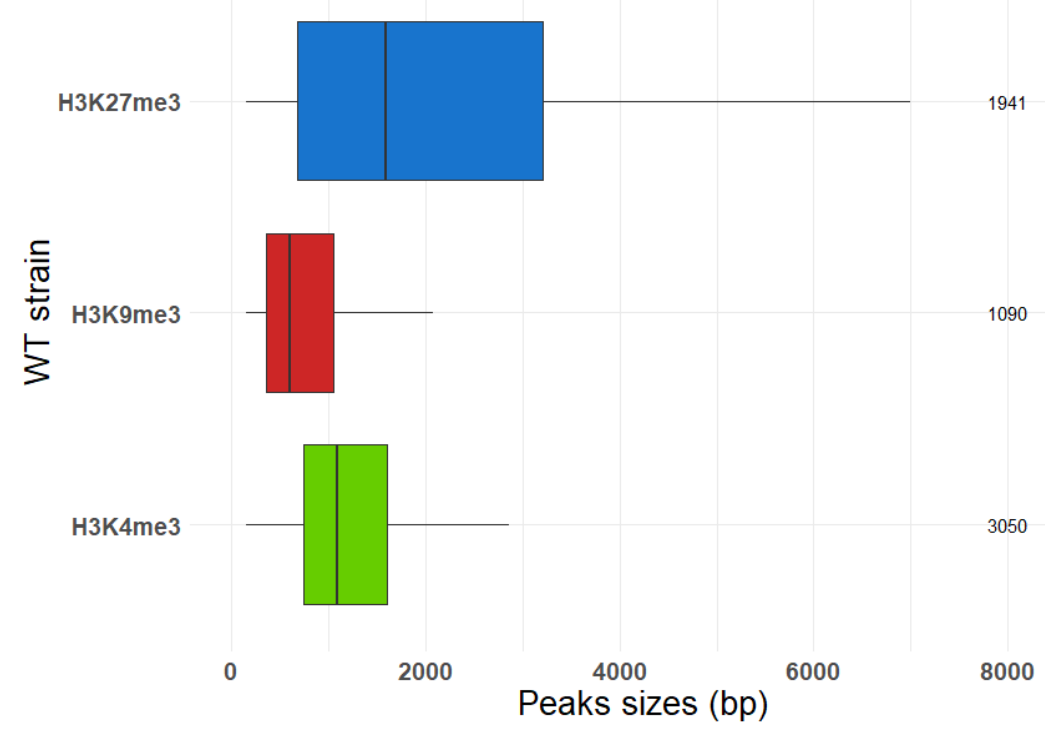

Fig. 3 B
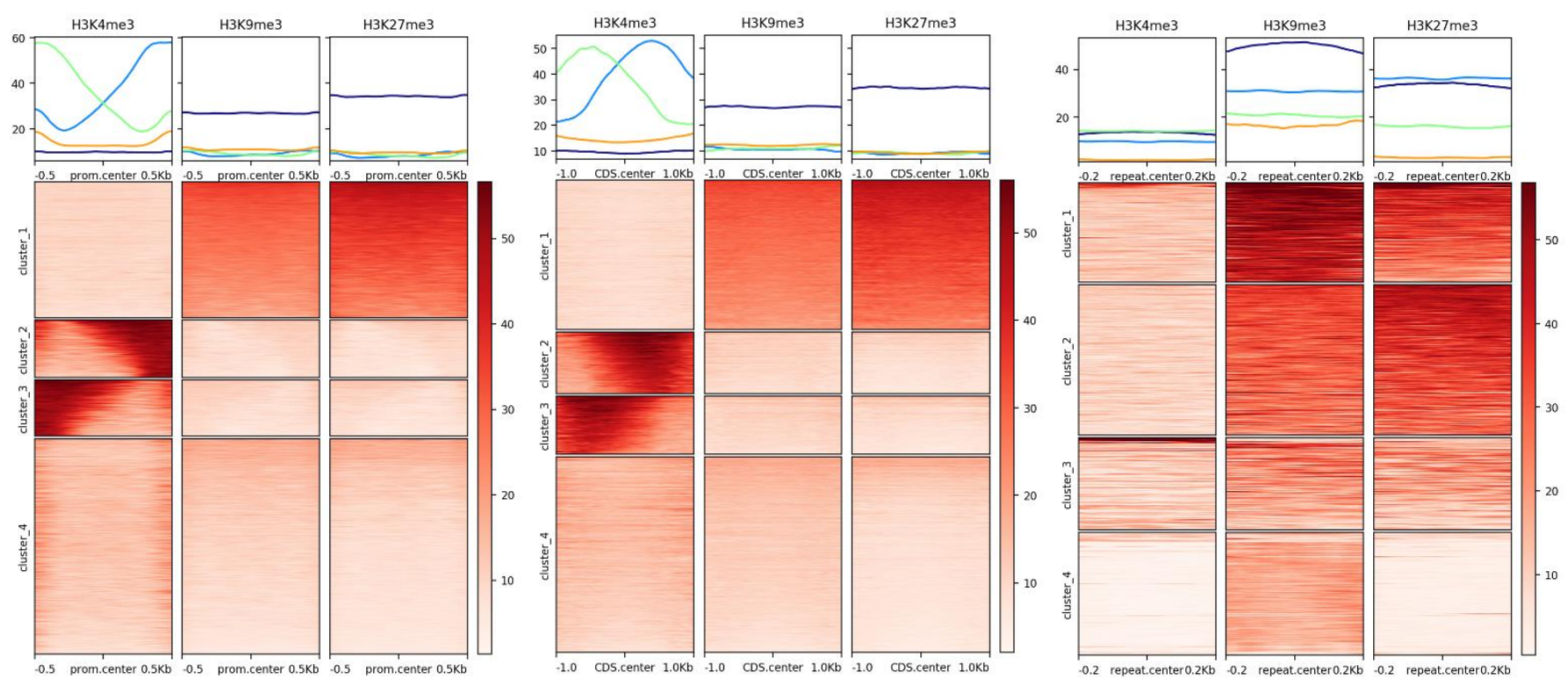

Fig. $3 \mathrm{C}$

Fig. $3 \mathrm{D}$
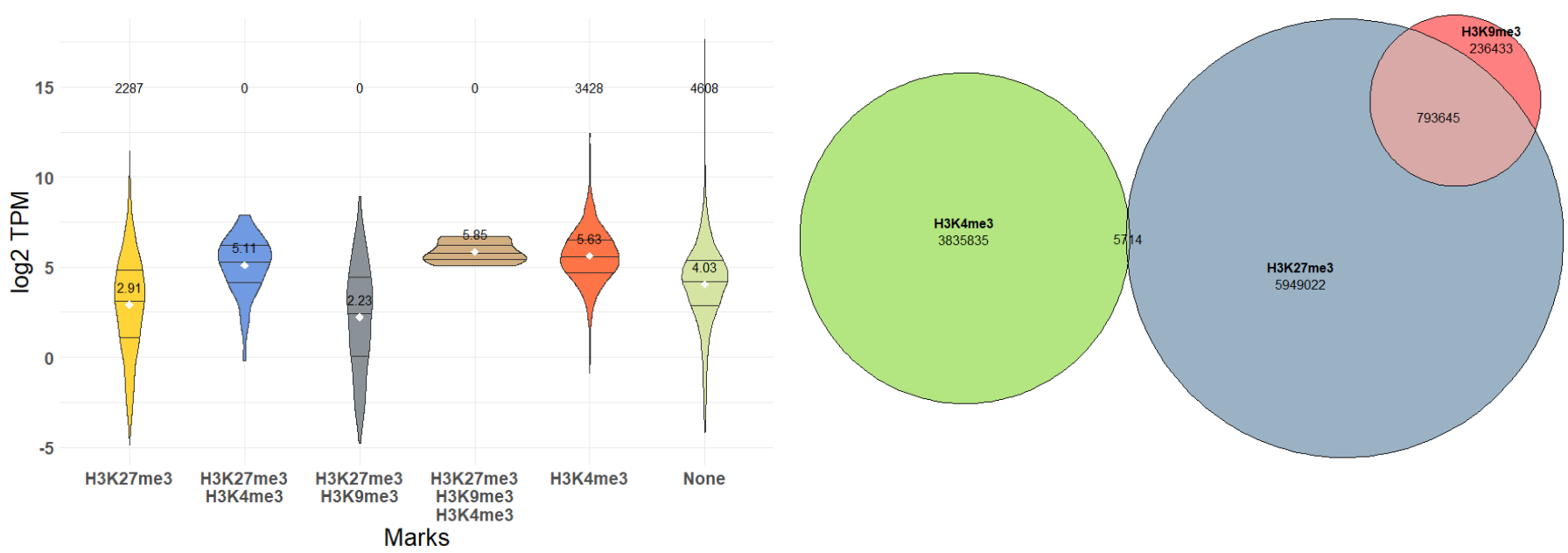
Fig. $5 \mathrm{~A}$

Fig. 5 B
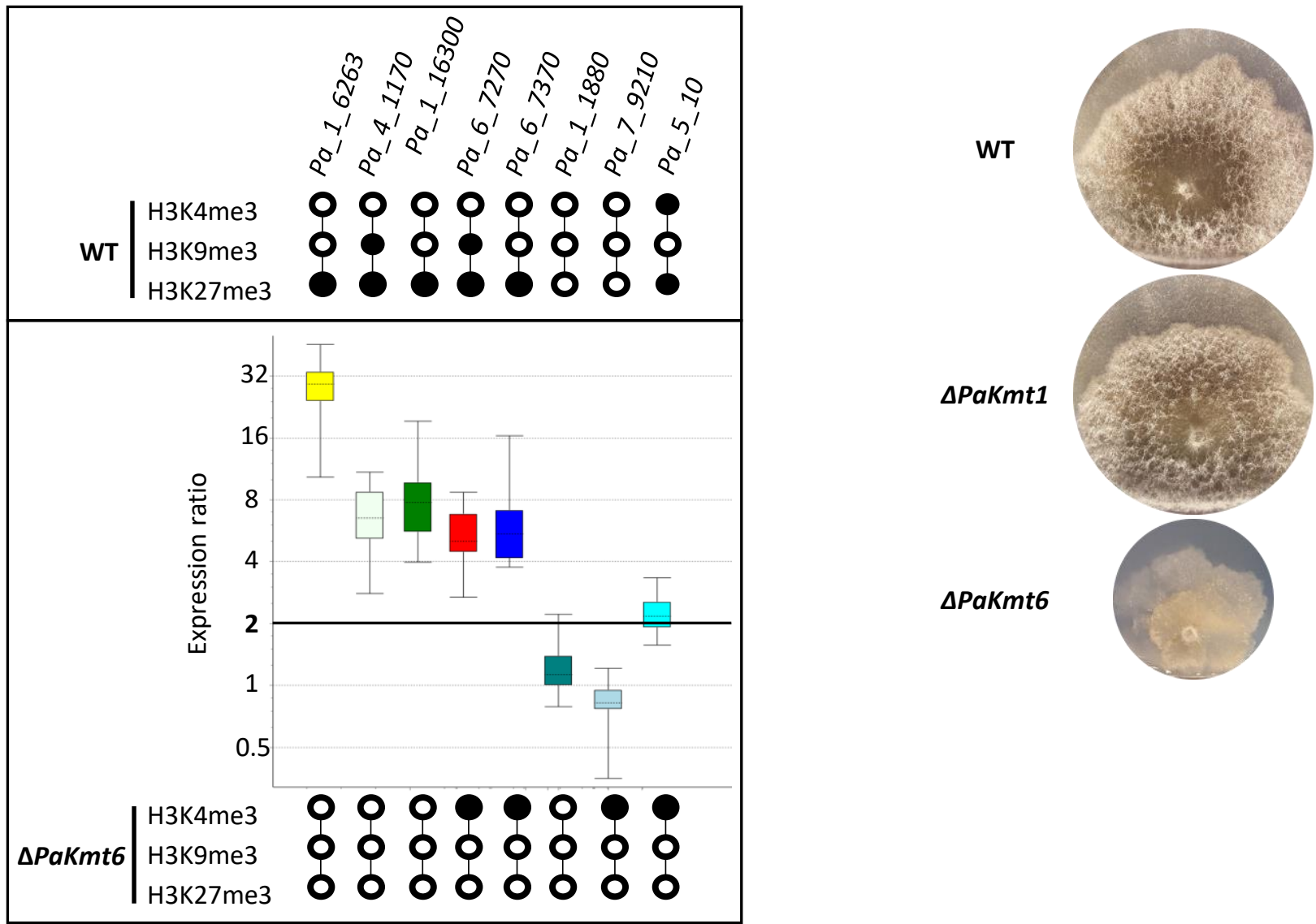

Fig. $5 \mathrm{C}$

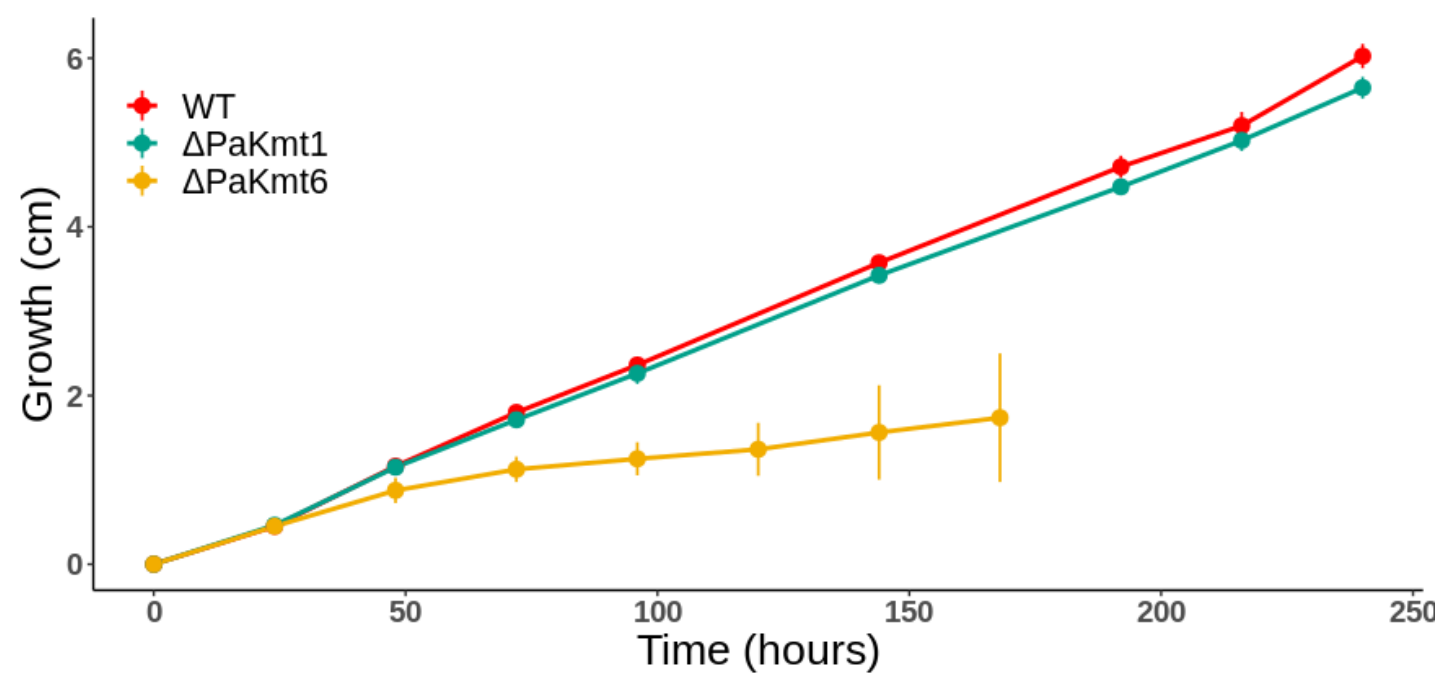


Fig. $6 \mathrm{~A}$

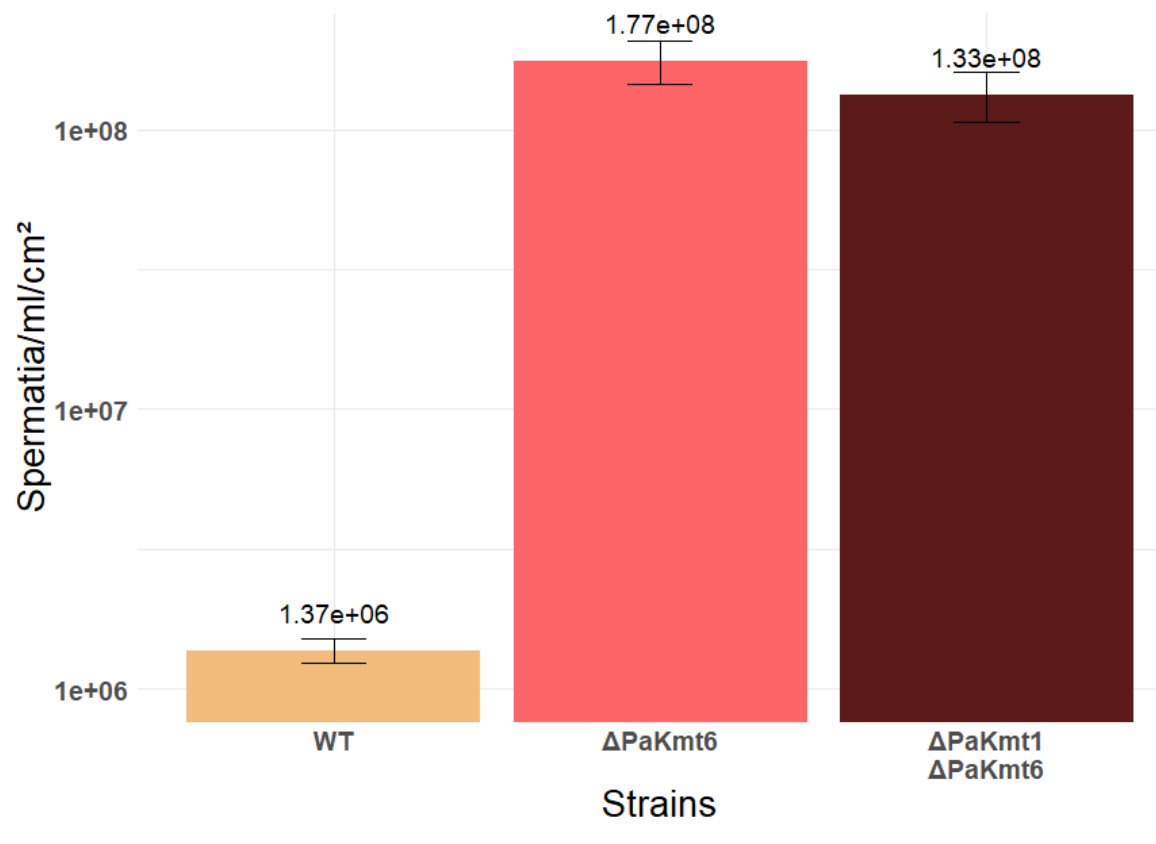

Fig. $6 \mathrm{~B}$

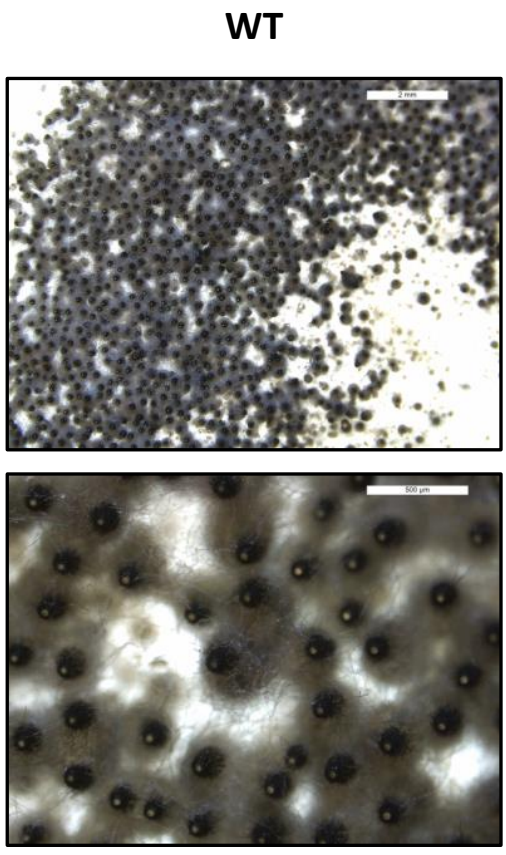

DPaKmt6
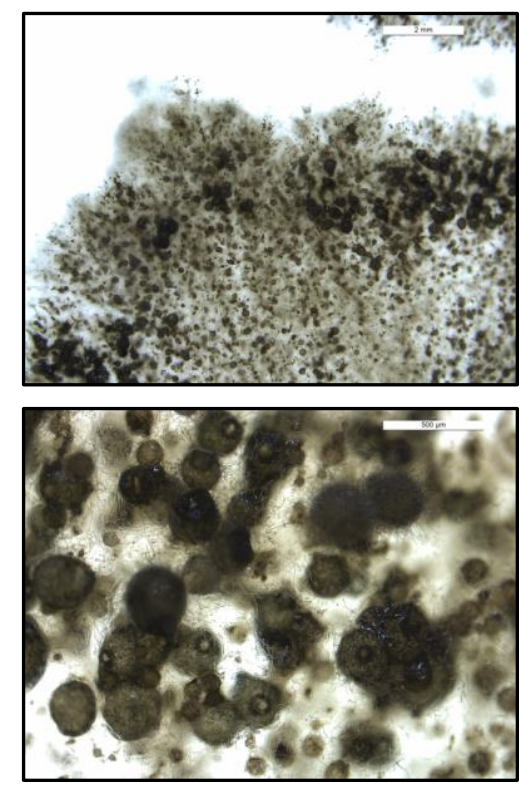
Fig. $7 \mathrm{~A}$

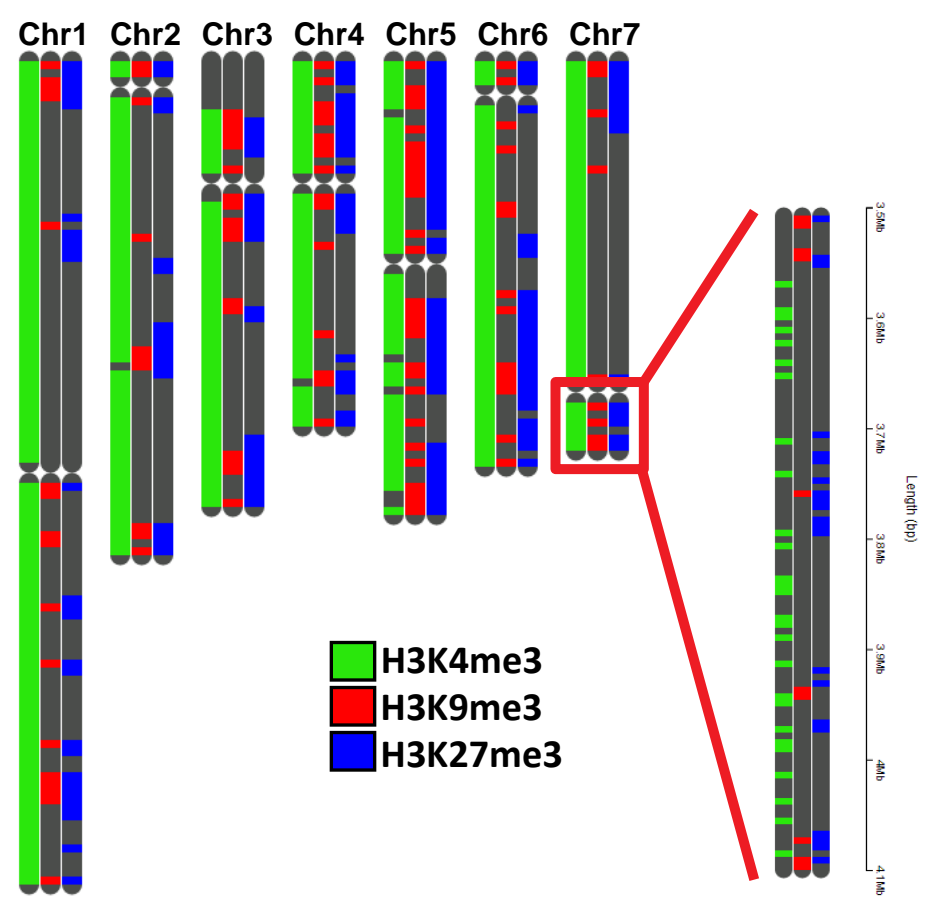

Fig. 7 B
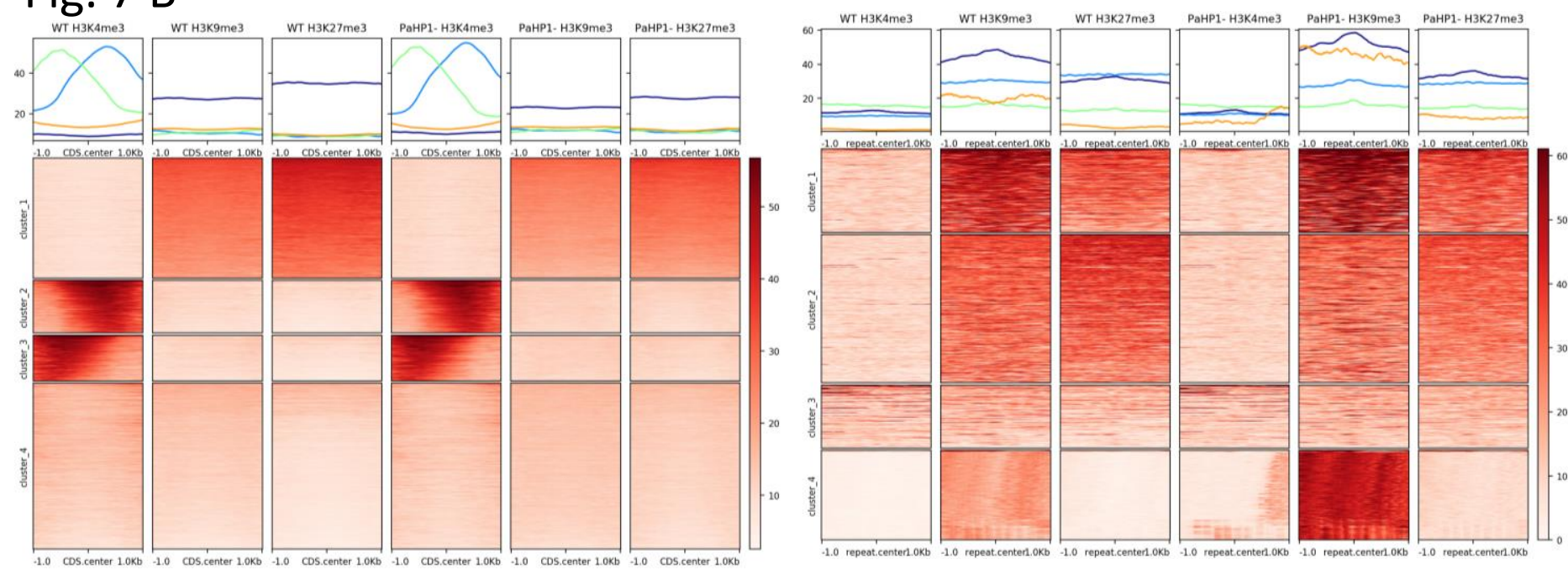

Fig. $7 \mathrm{C}$

8000
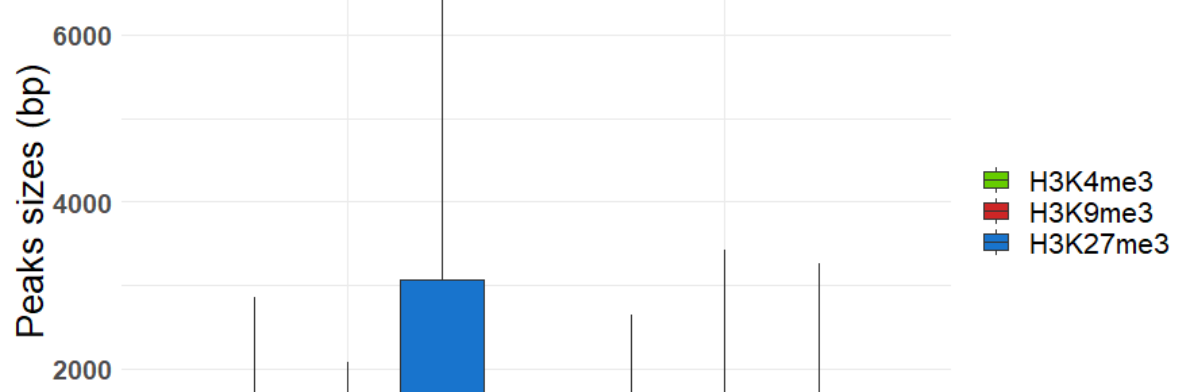
Fig. 8

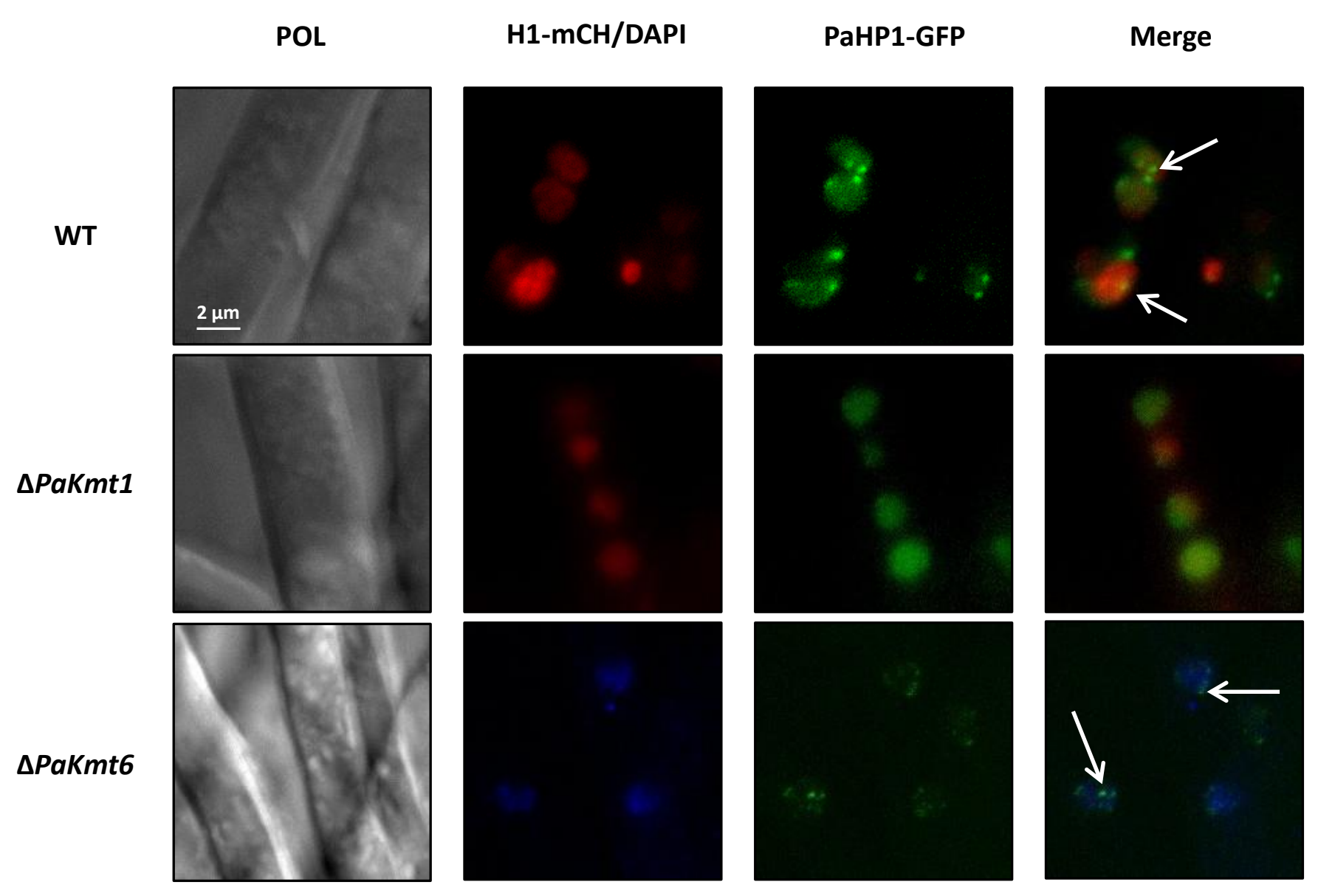




\section{Table 1}

\begin{tabular}{|c|c|c|c|}
\hline & $11^{\circ} \mathrm{C}$ & $27^{\circ} \mathrm{C}$ & $35^{\circ} \mathrm{C}$ \\
\hline$W T$ & 100 & 100 & 100 \\
\hline$\triangle P a K m t 1$ & 72.4 & 94.2 & 91.8 \\
\hline$\triangle$ PaKmt1 -PaKmt1+- & 104 & 100 & 99.7 \\
\hline$\Delta$ PaKmt6 & ND & $\begin{array}{c}\text { Irregular slow } \\
\text { growth }\end{array}$ & Irregular slow growth \\
\hline$\Delta$ PaKmt6 -PaKmt6 ${ }^{+}-$ & 98.3 & 99.0 & 99.3 \\
\hline$\Delta$ PaKmt1 $\triangle$ PaKmt6 & ND & $\begin{array}{c}\triangle P a K m t 6 \\
\text { aggravated }\end{array}$ & $\triangle P a K m t 6$ \\
\hline$\triangle P a H P 1$ & 65.7 & 76.2 & 74.9 \\
\hline$\triangle P a H P 1-P a H P 1^{+}-$ & 99.1 & 101 & 98.5 \\
\hline$\triangle P a K m t 1 \triangle P a P H 1$ & 60.7 & 89.9 & 80.7 \\
\hline$\triangle P a P H 1 \triangle P a K m t 6$ & ND & $\begin{array}{c}\triangle \text { PaKmt6 } \\
\text { aggravated }\end{array}$ & $\triangle$ PaKmt6 \\
\hline$\triangle P a K m t 1 \triangle P a P H 1 \triangle P a K m t 6$ & ND & $\begin{array}{c}\triangle P a K m t 6 \\
\text { aggravated }\end{array}$ & $\triangle P a K m t 6$ \\
\hline
\end{tabular}




\section{Table 2}

Germination efficiency

\begin{tabular}{r|c}
$W T$ & $92.8+/-4.26$ \\
$\Delta P a K m t 1$ & $W T$ \\
$\Delta P a K m t 6$ & $29.3+/-0.44$ \\
$\Delta P a K m t 6-P a K m t 6^{+}-$ & $W T$ \\
$\Delta P a K m t 1 \Delta P a K m t 6$ & $11.4+/-3.54$ \\
$\Delta P a H P 1$ & $W T$ \\
$\Delta P a K m t 1 \Delta P a P H 1$ & $W T$ \\
$\Delta P a P H 1 \Delta P a K m t 6$ & $25.2+/-2.31$ \\
$\Delta P a K m t 1 \Delta P a P H 1$ & $21.1+/-11.2$ \\
$\Delta P a K m t 6$ &
\end{tabular}


Table 3

\begin{tabular}{|c|c|c|c|c|c|}
\hline Species & & KMT1 mutant & кMT6 mutant & HP1 mutant & References \\
\hline \multirow{4}{*}{ Podospora anserina } & Growth \& development & $\begin{array}{l}\text { Reduced production of aerial hyphae } \\
\text { Slight growth defect }\end{array}$ & $\begin{array}{l}\text { Very poor growth } \\
\text { Less pigmentation }\end{array}$ & $\begin{array}{l}\text { Reduced production of aerial hyphae } \\
\text { Slight growth defect }\end{array}$ & This study \\
\hline & & Delay in resuming vegetative growth & $\begin{array}{l}\text { Overproduction of conidia } \\
\text { Mishonaned fructification }\end{array}$ & & \\
\hline & & & $\begin{array}{l}\text { Mishsappeded fructification } \\
\text { Defect in spore germination }\end{array}$ & & \\
\hline & $\begin{array}{l}\text { Chromatin \& regulation } \\
\text { ChIP-seq }\end{array}$ & Loss of H3K9me3 & Loss of H3K27me3 and H3K9me3 3 & Broader H3K9me3 domains & \\
\hline \multirow{3}{*}{ Neurospora crassa } & Growth \& development & $\begin{array}{l}\text { Reduced growth } \\
\text { Affected in sexual development }\end{array}$ & None & $\begin{array}{l}\text { Reduced growth } \\
\text { Affected in sexual development }\end{array}$ & $\begin{array}{l}\text { Basenko et al. 2015, Freitag et al. } 2004 \\
\text { Jamieson et al. 2013; } 2016 \\
\text { Klocko et al. 2016 }\end{array}$ \\
\hline & Chromatin \& regulation & Affected in DNA methylation & Loss of $\mathrm{H} 3 \mathrm{~K} 27 \mathrm{me} 2 / 3$ & Affected in DNA methylation & \\
\hline & & $\begin{array}{l}\text { Relocalization of H3K27me3 } \\
\text { Loss of HKK9me3 } \\
\text { H3K27me3 relocation and diminution }\end{array}$ & Altered in chromosome conformation & 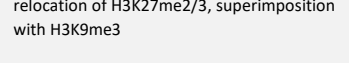 & \\
\hline \multirow{3}{*}{$\begin{array}{c}\begin{array}{c}\text { Fusarium } \\
\text { verticilloides }\end{array} \\
\text {. }\end{array}$} & Growth \& development & $\begin{array}{l}\text { Defects in conidiation, perithecium } \\
\text { production and fungal virulence }\end{array}$ & N/A & N/A & Gu et al. 2017 \\
\hline & & Overpigmentation & & & \\
\hline & Chromatin \& regulation & N/A & N/A & N/A & \\
\hline \multirow{2}{*}{$\begin{array}{l}\text { Fusarium } \\
\text { graminearum }\end{array}$} & Growth \& development & N/A & $\begin{array}{l}\text { Growth defects } \\
\text { Sterility } \\
\text { Overpigmentation } \\
\text { Reduced virulence }\end{array}$ & Overpigmentation & $\begin{array}{l}\text { Connolly, Smith, and Freitag } 2013 \\
\text { Reyes-Dominguez et al. } 2012\end{array}$ \\
\hline & $\begin{array}{l}\text { Chromatin \& regulation } \\
\text { ChIP-seq }\end{array}$ & N/A & $\begin{array}{l}\text { Loss of H3K27me3 } \\
\text { Constitutive expression of genes encoding mycotoxins } \\
\text { pigments-producing enzymes and other SM }\end{array}$ & $\begin{array}{l}\text { Enhanced activity of activity of biosynthetic } \\
\text { cluster genes }\end{array}$ & \\
\hline \multirow{2}{*}{ Epichloë festucae } & Growth \& development & $\begin{array}{l}\text { Reduced vegetative growth } \\
\text { Deregulation of symbiosis }\end{array}$ & Deregulation of symbiosis & Deregulation of symbiosis & $\begin{array}{l}\text { Chujo and Scott, } 2014 \\
\text { Chujo et al. } 2019 \text {. }\end{array}$ \\
\hline & $\begin{array}{l}\text { Chromatin \& regulation } \\
\text { ChIP-APCR }\end{array}$ & $\begin{array}{l}\text { Derepression of symbiosis-specific alkal oid } \\
\text { genes clusters }\end{array}$ & Derepression of symbiosis-specific alkaloid genes clusters & $\begin{array}{l}\text { Derepression of symbiosis-specific alkaloid } \\
\text { genes clusters }\end{array}$ & \\
\hline \multirow[t]{2}{*}{ Magnaporthe oryzae } & Growth \& development & $\begin{array}{l}\text { Reduced vegetative growth } \\
\text { Reduced virulence }\end{array}$ & $\begin{array}{l}\text { Reduced vegetative growth } \\
\text { Severe reduction in conidiation } \\
\text { Slight defects in appressorium formation } \\
\text { Reduction in pathogenicity }\end{array}$ & N/A & Pham KTM et al. 2015 \\
\hline & $\begin{array}{l}\text { Chromatin \& regulation } \\
\text { WB }\end{array}$ & Loss of H3K9me3 & Loss of HзK27me3 & N/A & \\
\hline \multirow[b]{2}{*}{ Zymoseptoria tritici } & Growth \& development & $\begin{array}{l}\text { Reduced vegetative growth } \\
\text { Reduced virulence }\end{array}$ & Reduced virulence & N/A & Möller et al., 2019 \\
\hline & $\begin{array}{l}\text { Chromatin \& regulation } \\
\text { ChIP-seq }\end{array}$ & $\begin{array}{l}\text { Loss of H3K9me3 } \\
\text { TE activation and genomic rearrangements } \\
\text { H3K27mer } \\
\text { Redolocation } \\
\text { Retion of H3K27me3 }\end{array}$ & $\begin{array}{l}\text { Loss of HзКк27me3 } \\
\text { Increased stability of accessory chromosomes }\end{array}$ & N/A & \\
\hline \multirow[b]{2}{*}{ Aspergillus nidulans } & Growth \& development & No developmental phenotype & N/A & No developmental phenotype & Reyes-Dominguez et al. 2010 \\
\hline & Chromatin \& regulation & $\begin{array}{l}\text { Enhanced expression of a subset of } \\
\text { secondary metabolite }(S M) \text { clusters }\end{array}$ & N/A & $\begin{array}{l}\text { Enhanced expression of a subset of } \\
\text { secondary metabolite }(S M) \text { clusters }\end{array}$ & \\
\hline \multirow{2}{*}{ Aspergillus fumigatus } & Growth \& development & $\begin{array}{l}\text { Reduced vegetative growth } \\
\text { delayed conidiation }\end{array}$ & N/A & $\begin{array}{l}\text { Reduced vegetative growth and delayed } \\
\text { conidiation }\end{array}$ & Palmer et al. 2008 \\
\hline & Chromatin \& regulation & N/A & N/A & N/A & \\
\hline \multirow{2}{*}{$\begin{array}{l}\text { Leptosphaeria } \\
\text { maculans }\end{array}$} & Growth \& development & $\begin{array}{l}\text { Reduced vegetative growth } \\
\text { Reduced virulence }\end{array}$ & N/A & Reduced vegetative growth & Soyer et al. 2014 \\
\hline & $\begin{array}{l}\text { Chromatin \& regulation } \\
\text { ChIP-seq (WT only) }\end{array}$ & Up-regulation of pathogenicity-related genes & N/A & Up-regulation of pathogenicity-related genes & \\
\hline \multirow{2}{*}{ Penicillium oxalicum } & Growth \& development & $\mathrm{N} / \mathrm{A}$ & N/A & $\begin{array}{l}\text { Reduced vegetative growth } \\
\text { Delayed conidiation }\end{array}$ & Zhang et al. 2016 \\
\hline & Chromatin \& regulation & N/A & N/A & N/A & \\
\hline
\end{tabular}


Fig. S1 A

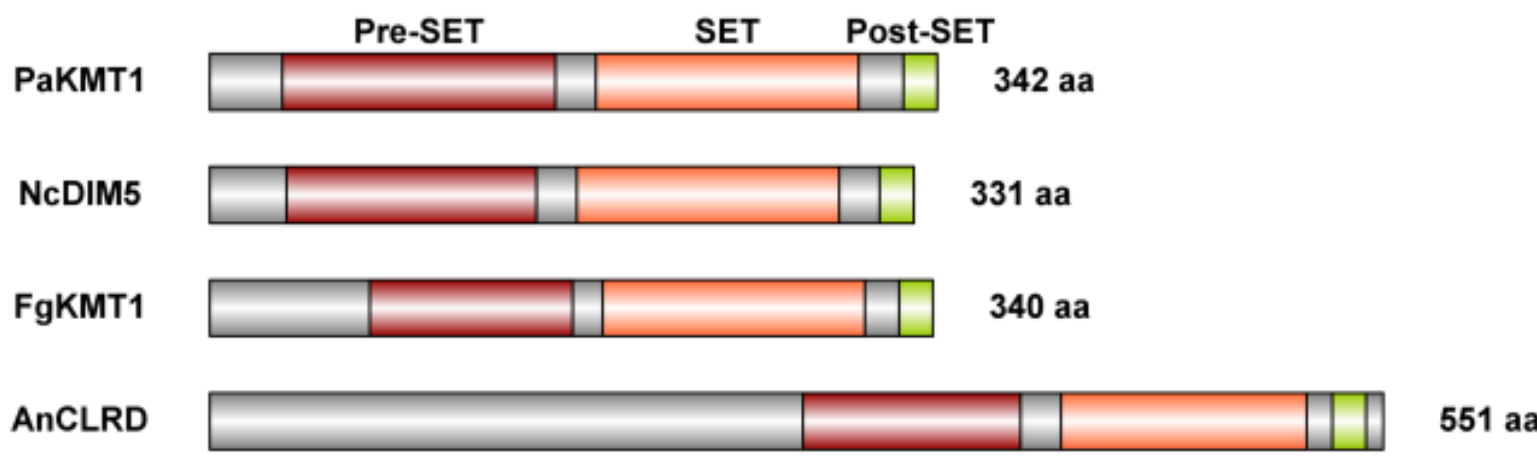

\section{Chromo}

\begin{tabular}{l||l|l|l|l|l|l|} 
SpCLR4 & & & & & & \\
4
\end{tabular}

DmSU(VAR)3-9

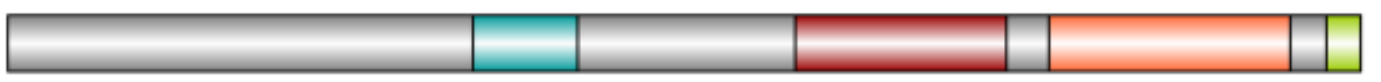

635 aa

MmSUV39H2

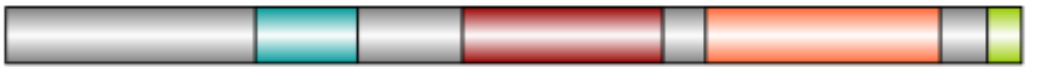

477 aa

SRA

AtSUVH4

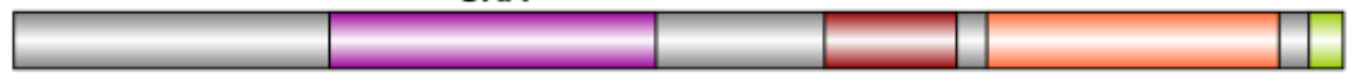

624 aa

Fig. S1 B

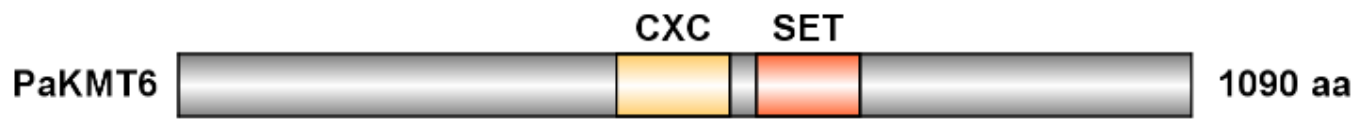

NcSET-7

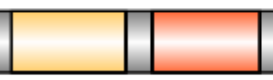

1611 aa

FgKMT6

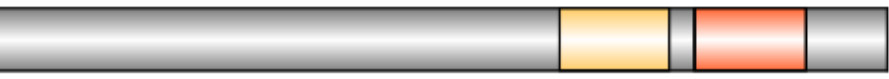

958 aa

EfKMT6
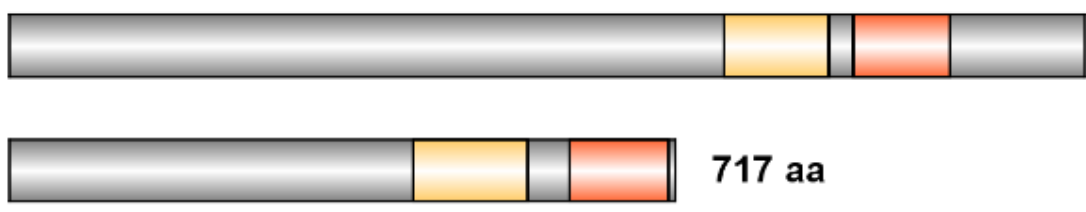

1158 aa

CnEzh2

717 aa

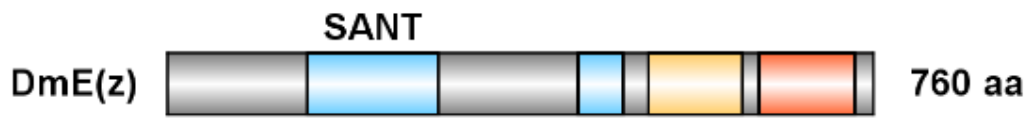

MmEZH2

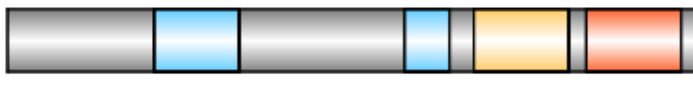

746 aa

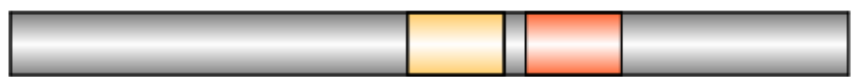


Fig. S1 C

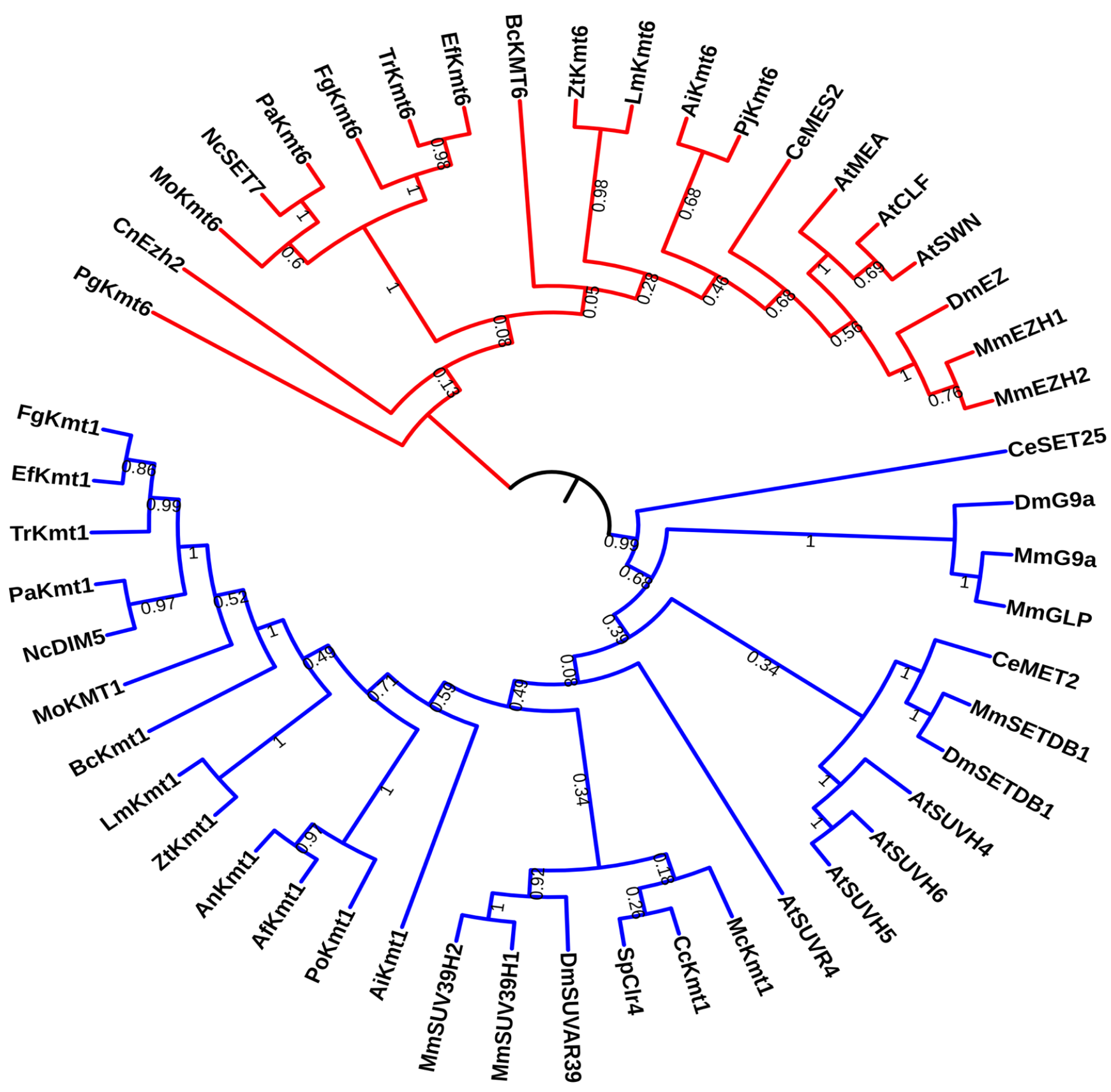


Fig. S2

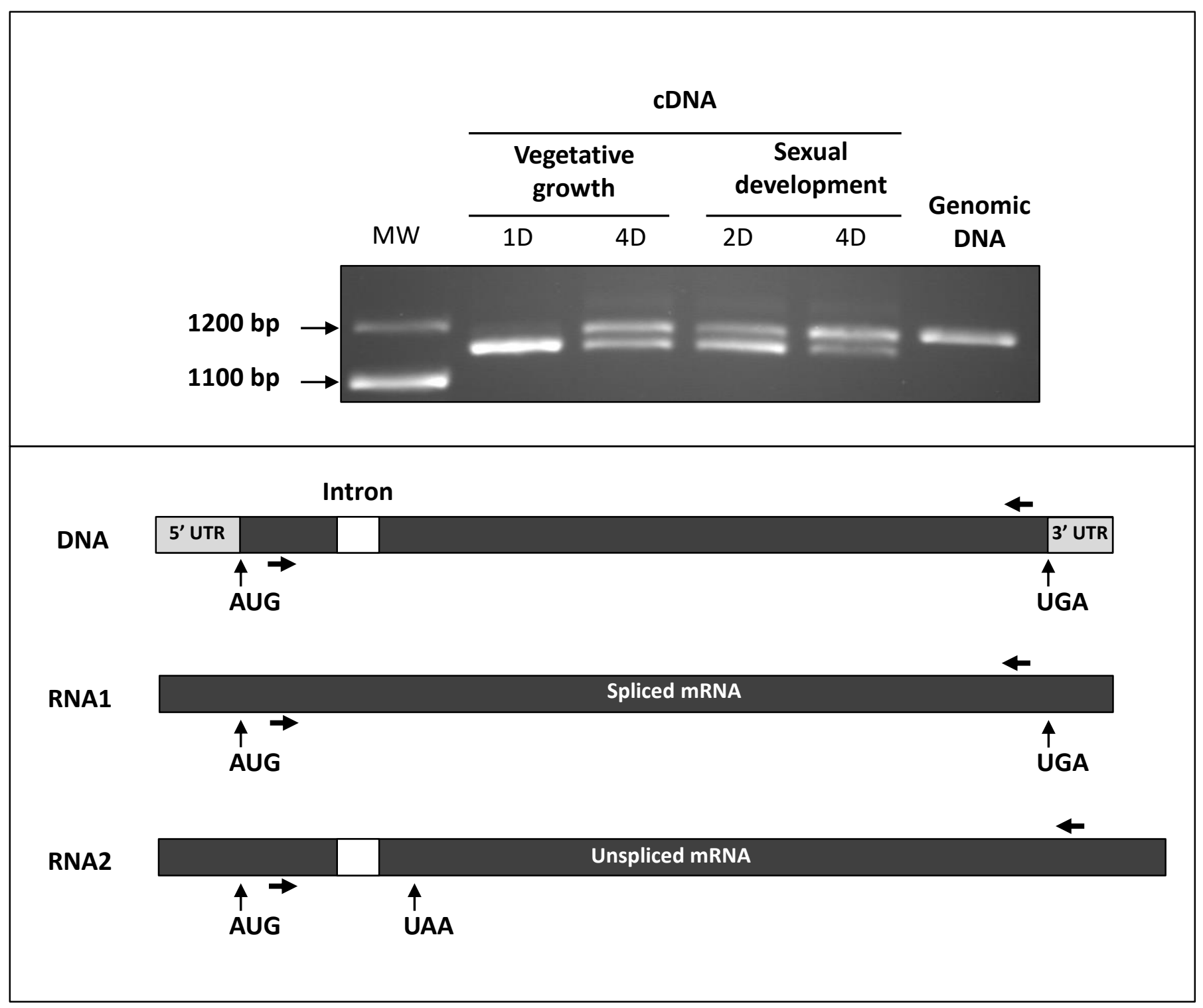


Fig. S3

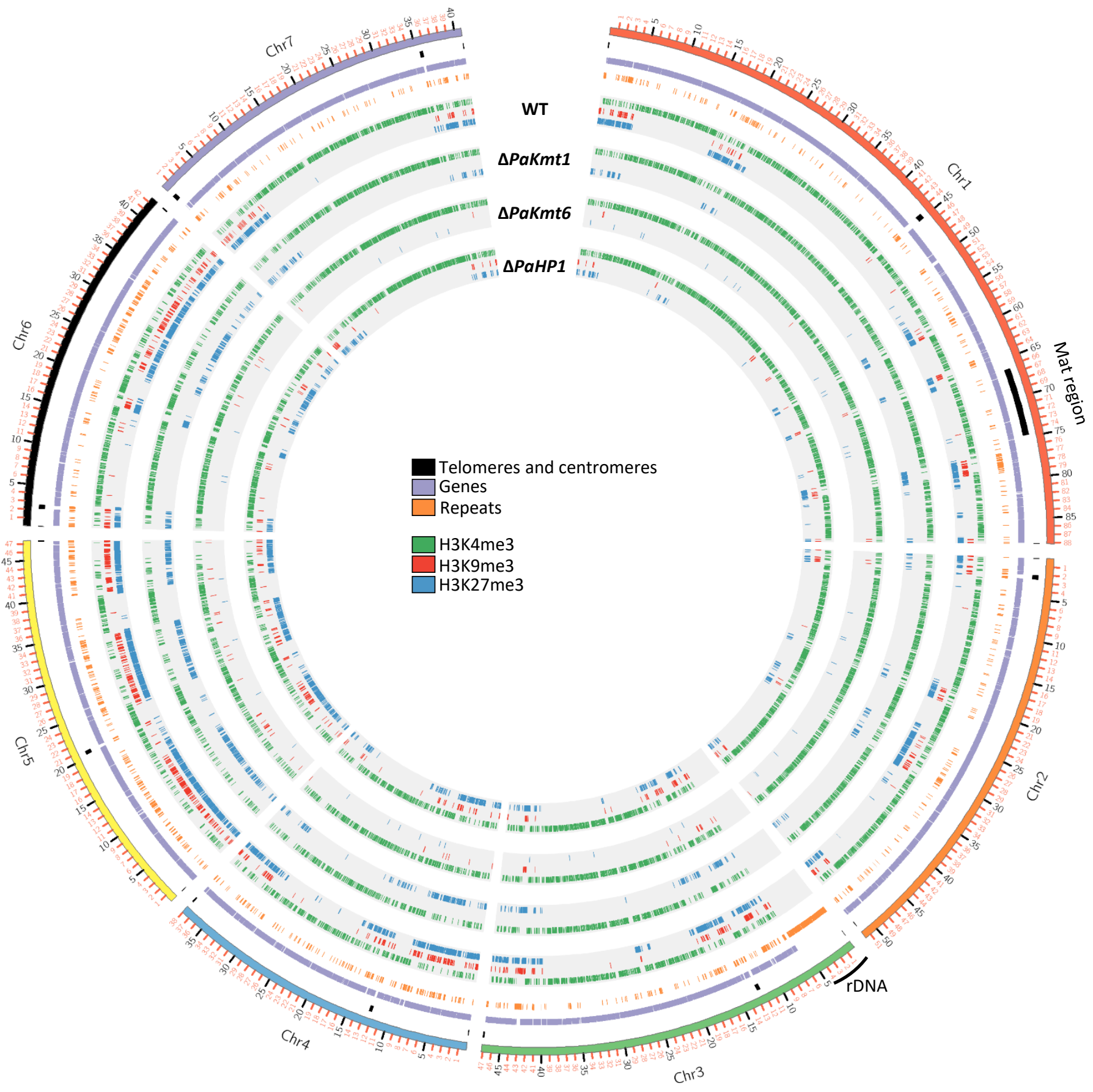




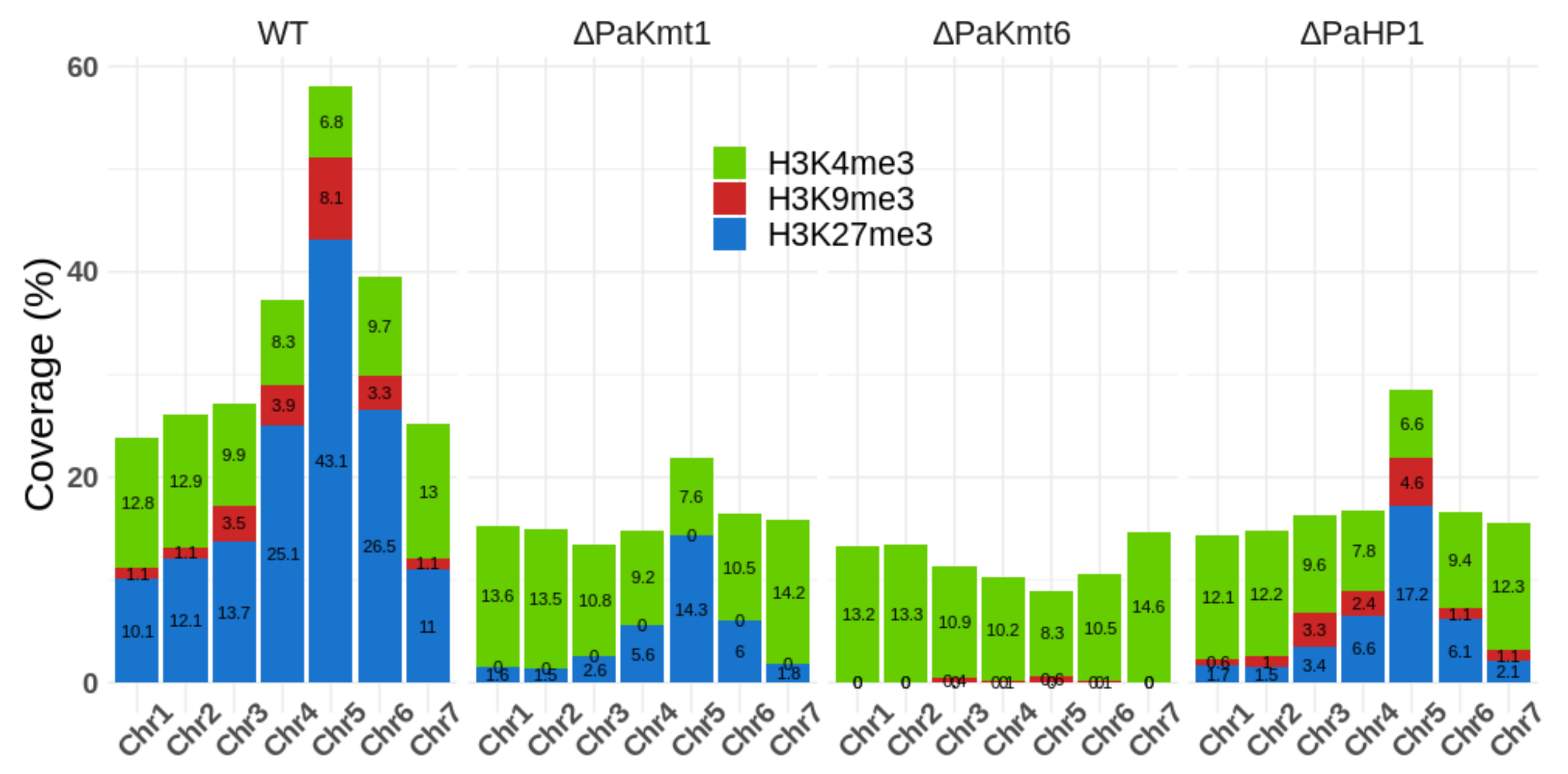


Fig. S5
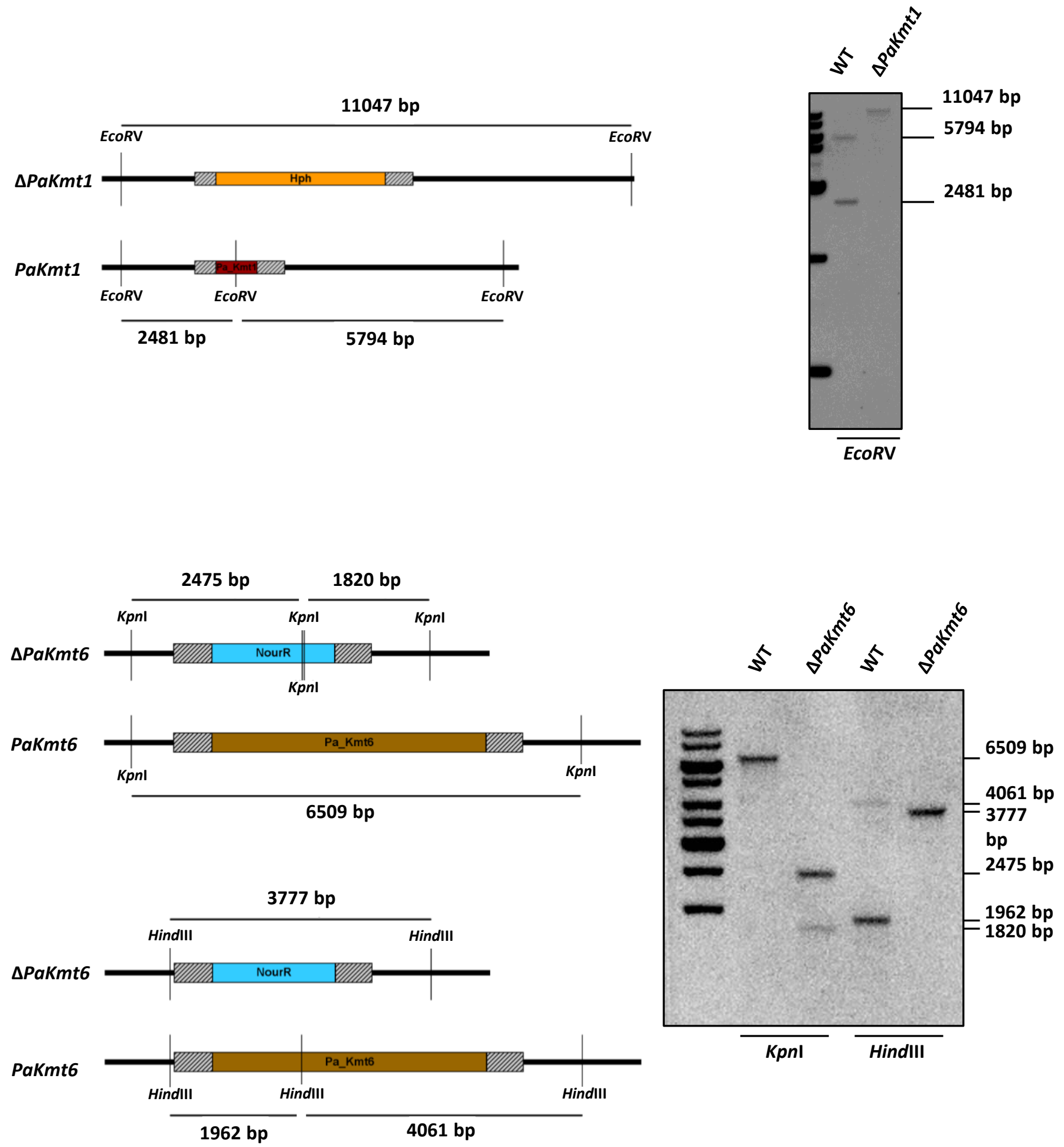
Fig. 56

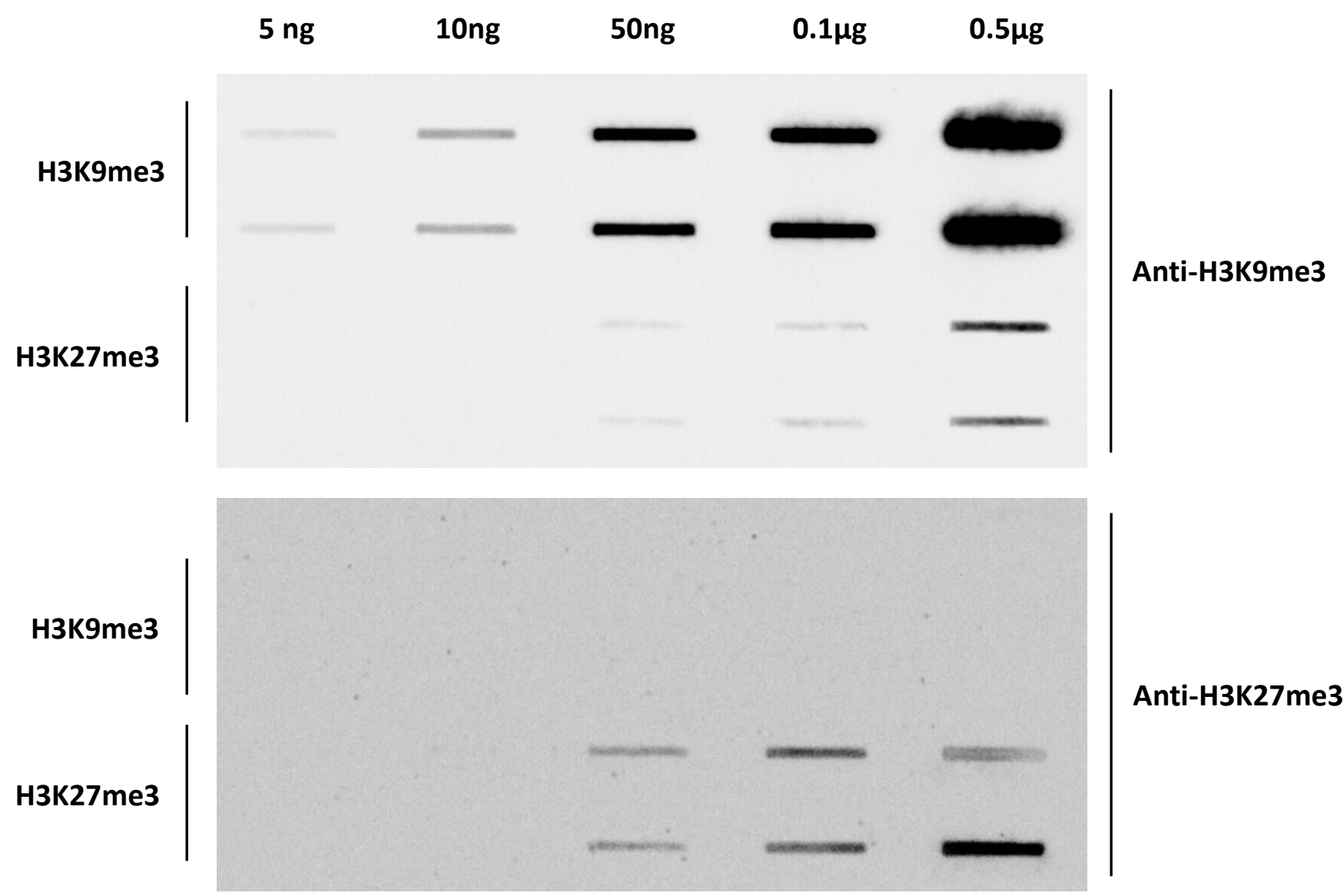


Fig. 57

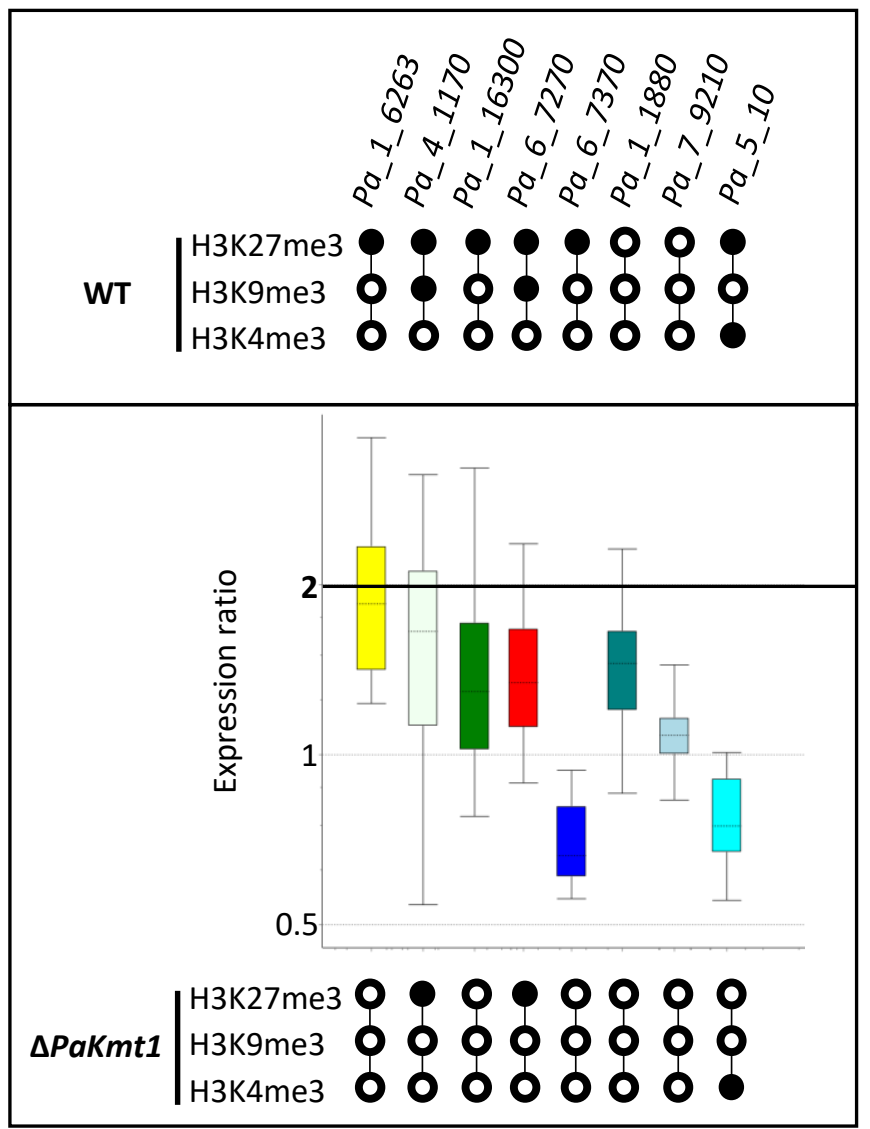


Fig. S8 A

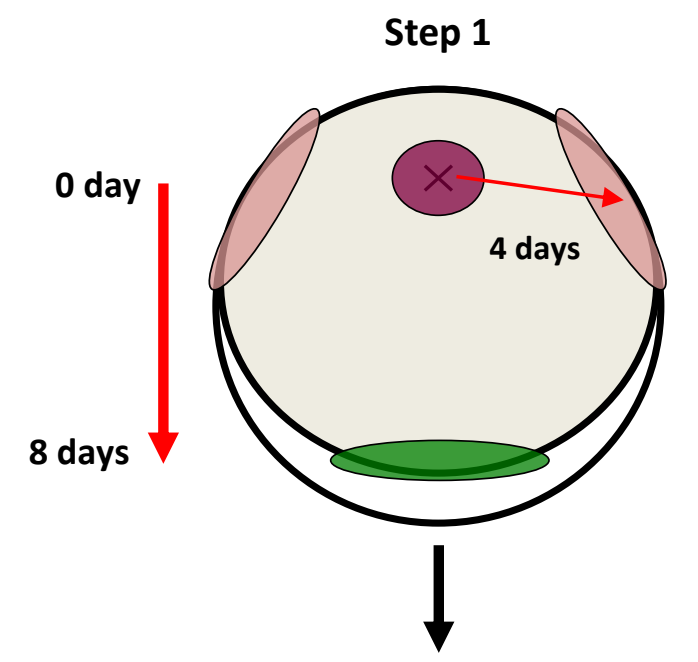

Step 1

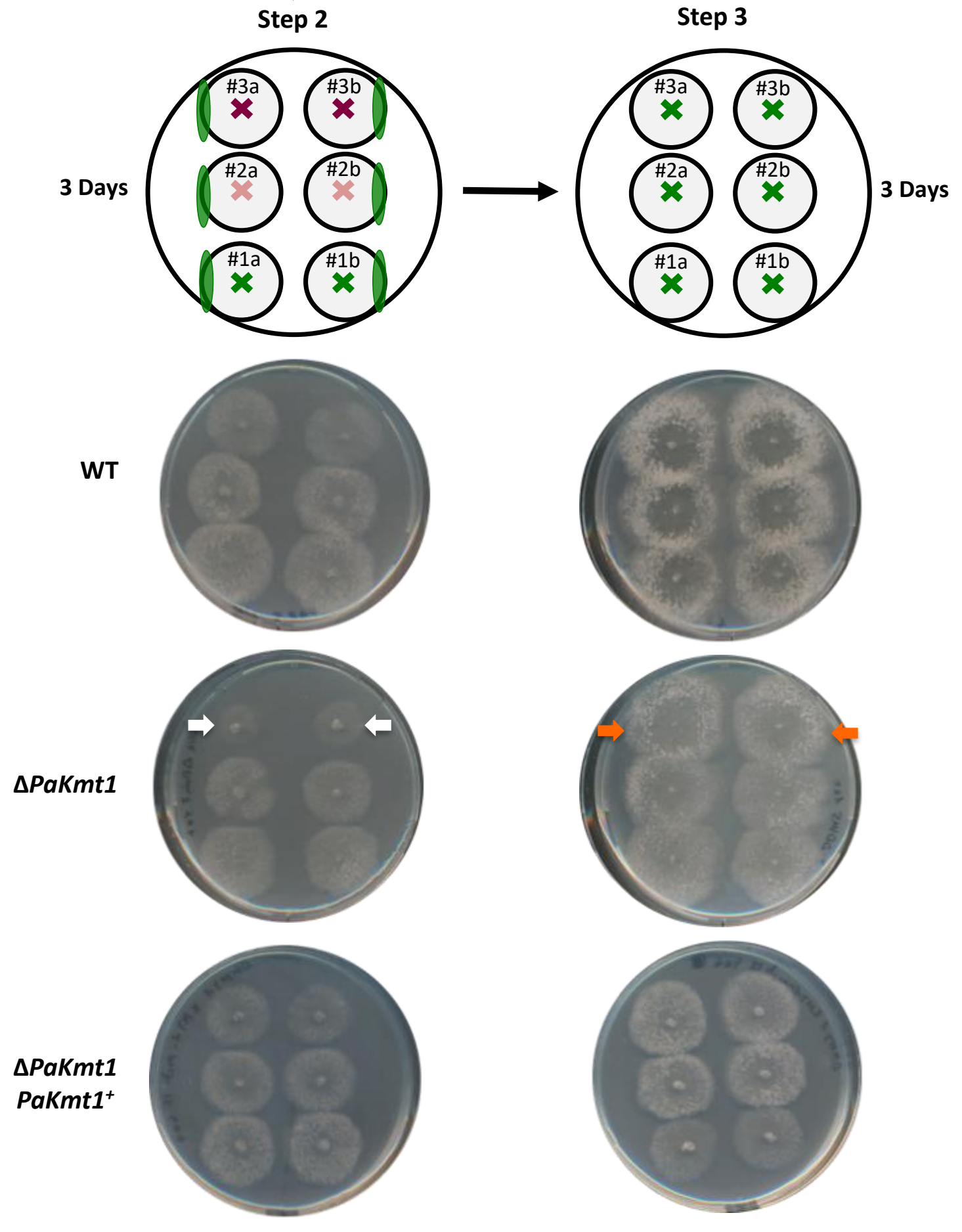


Fig. S8 B

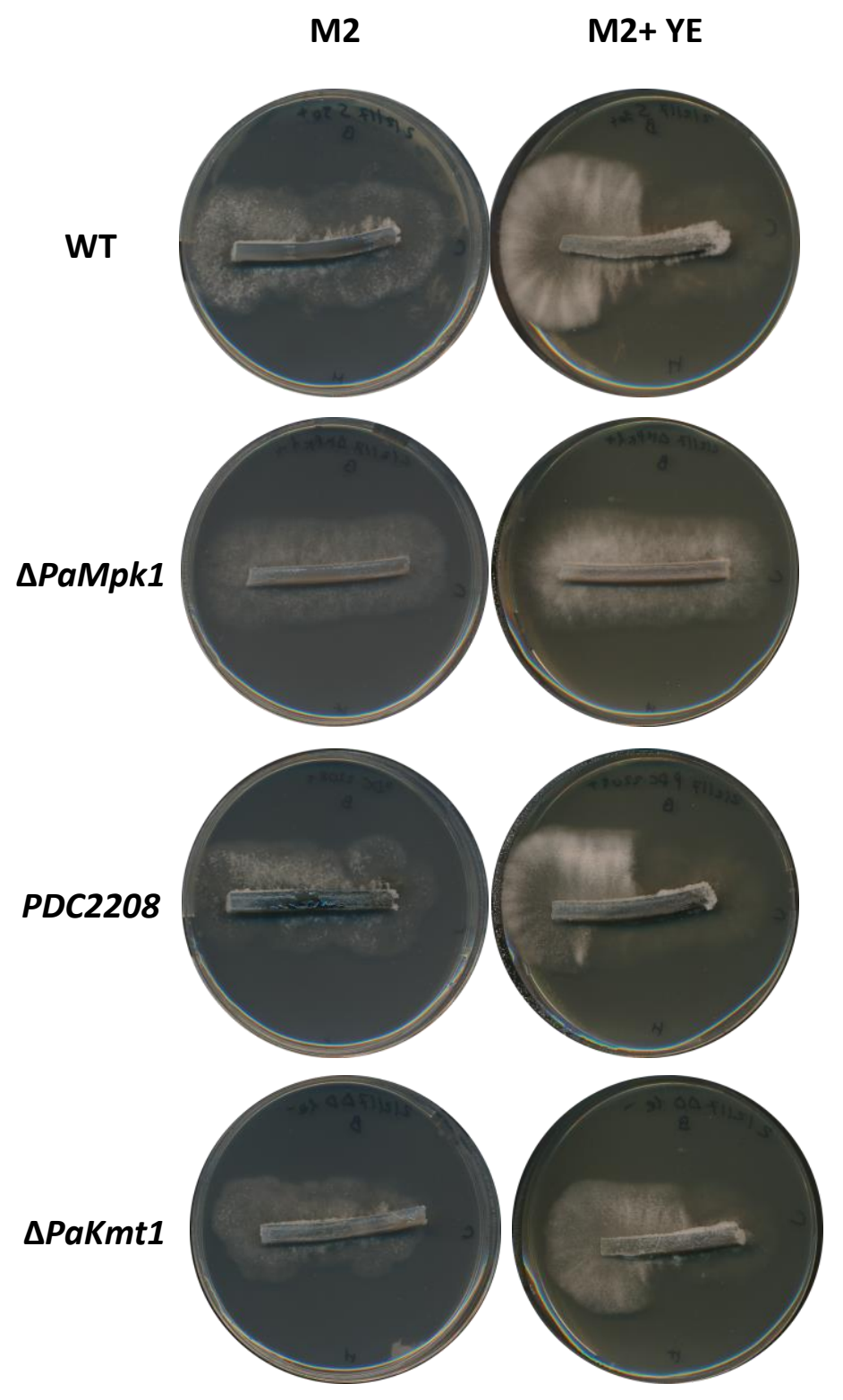


Fig. 59
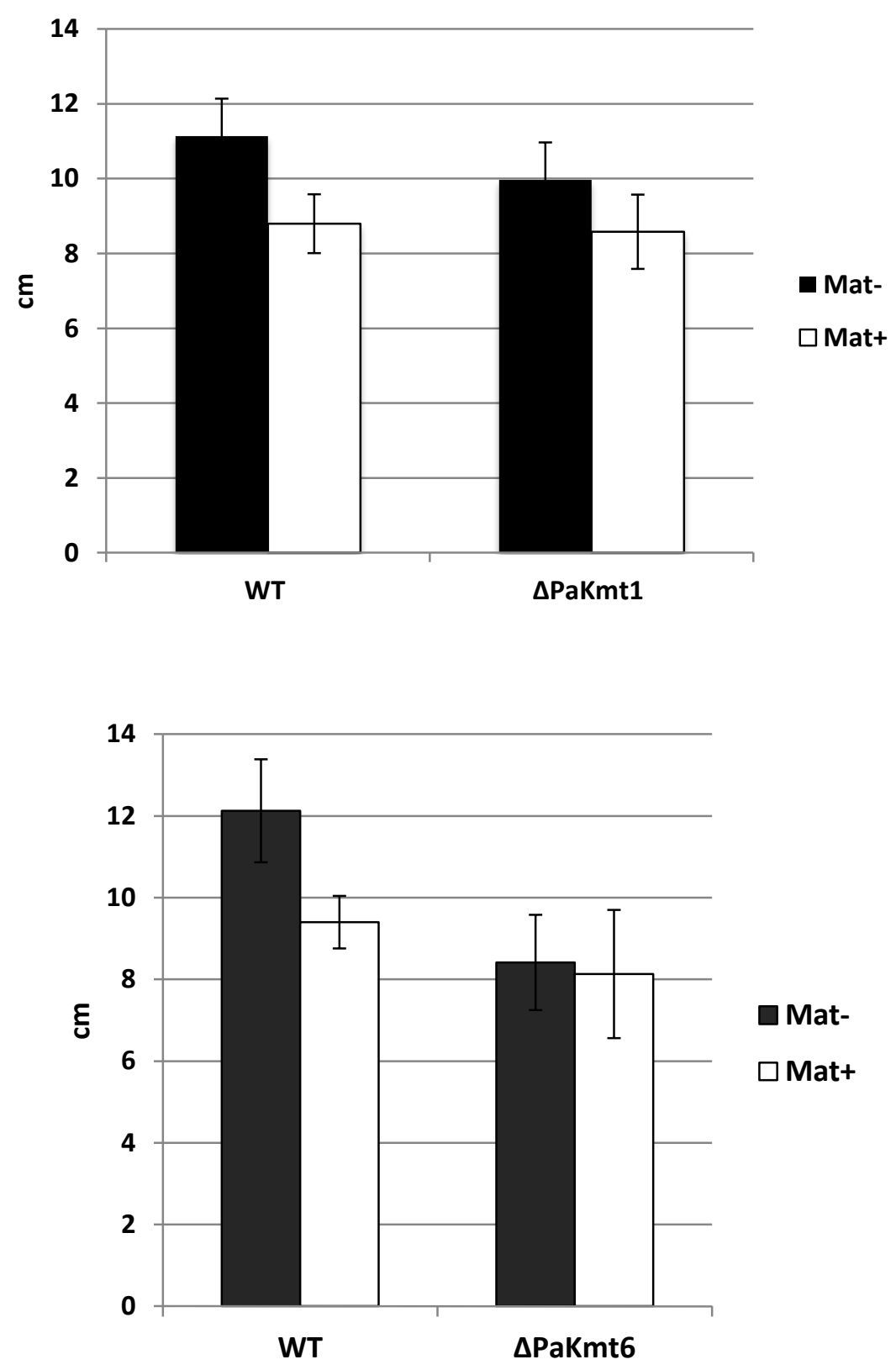
Fig. S10 A

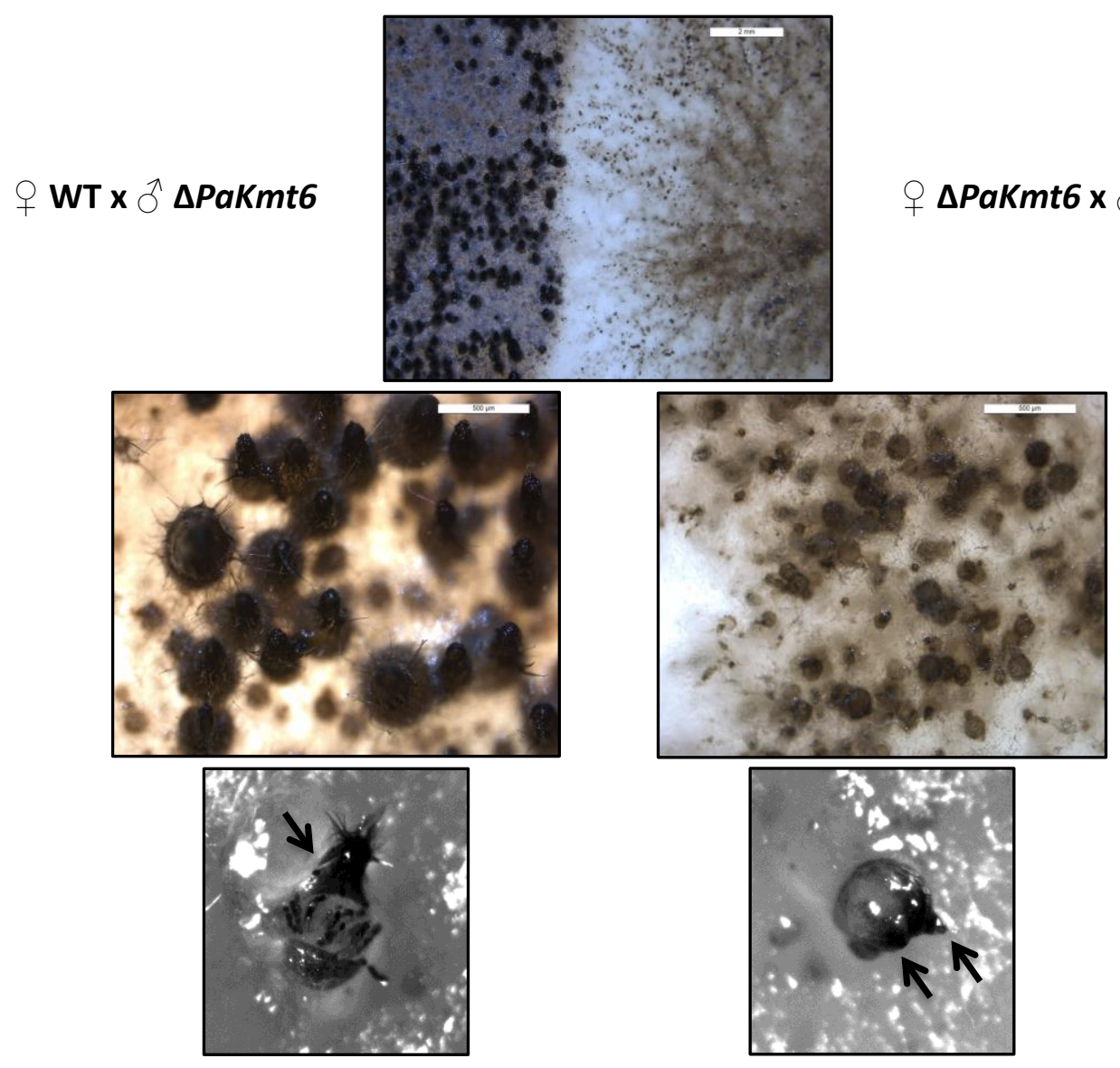

Fig. S10 B

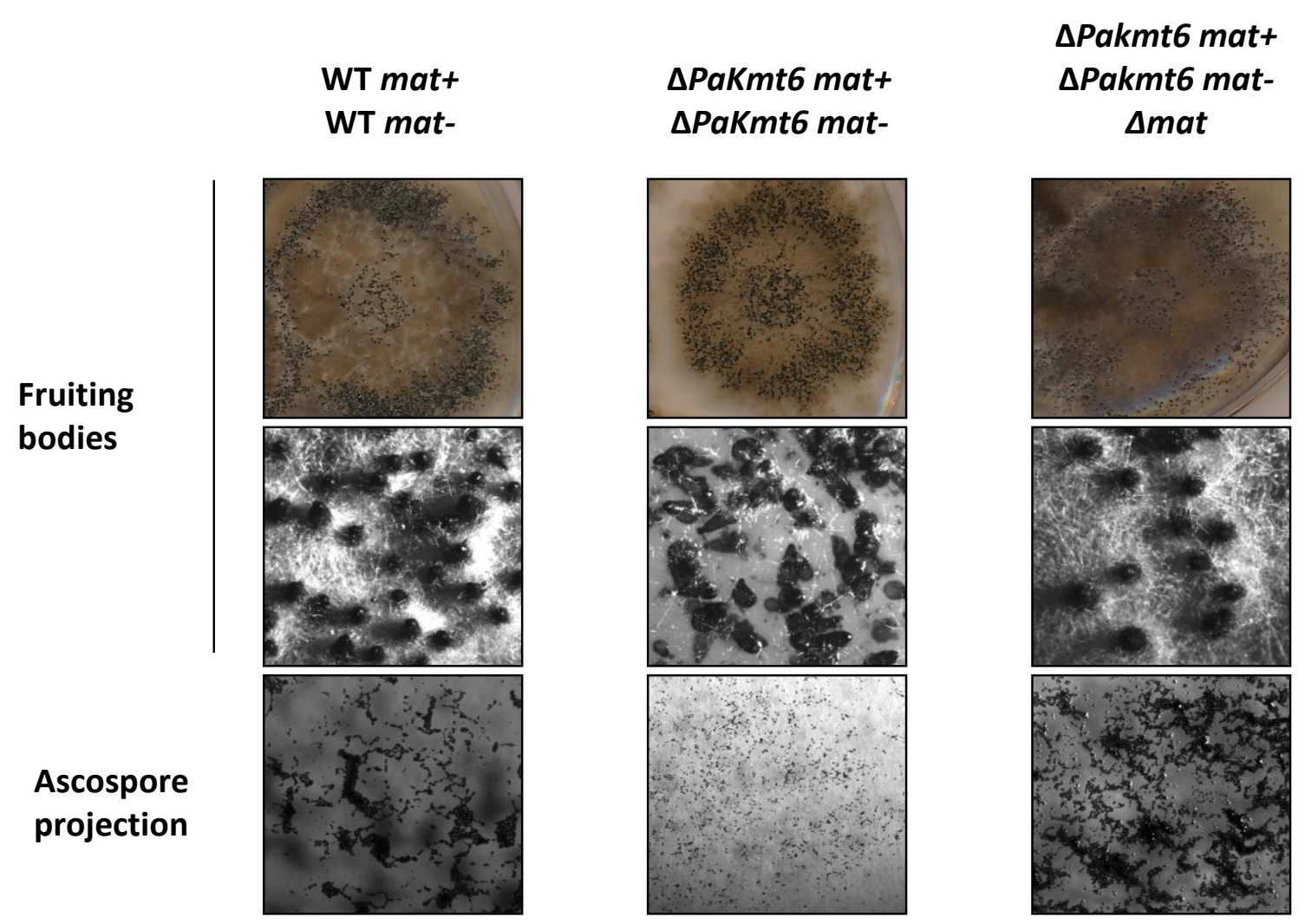


Fig. S11 A
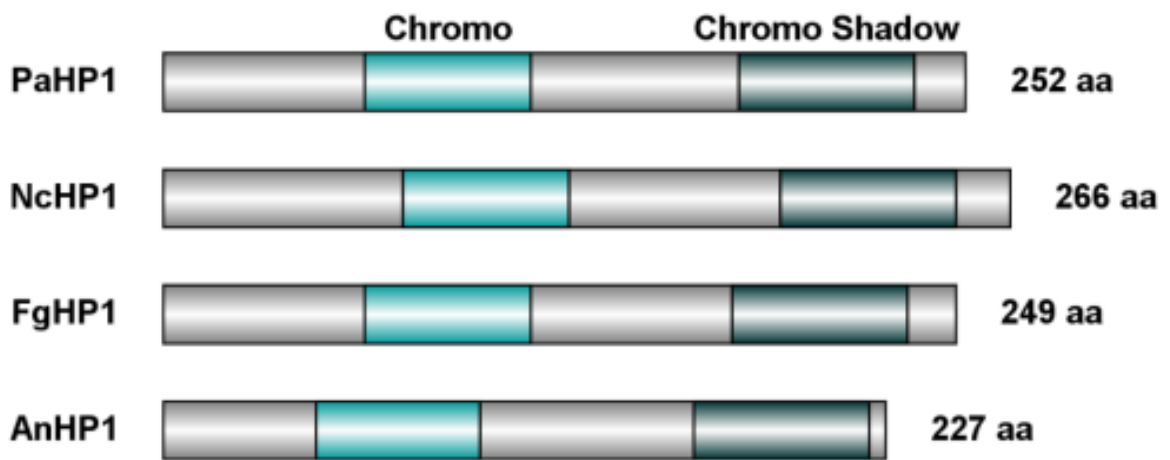

SpSwi6

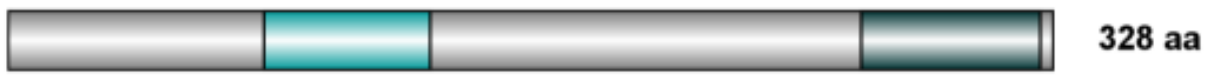

DmSU(VAR)205

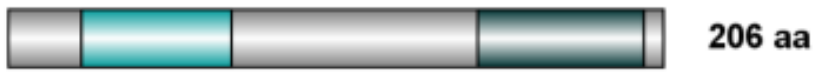

MmHP1a

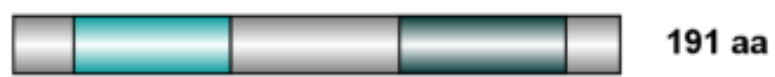

AtLHP1

435 aa 
Fig. S11 B

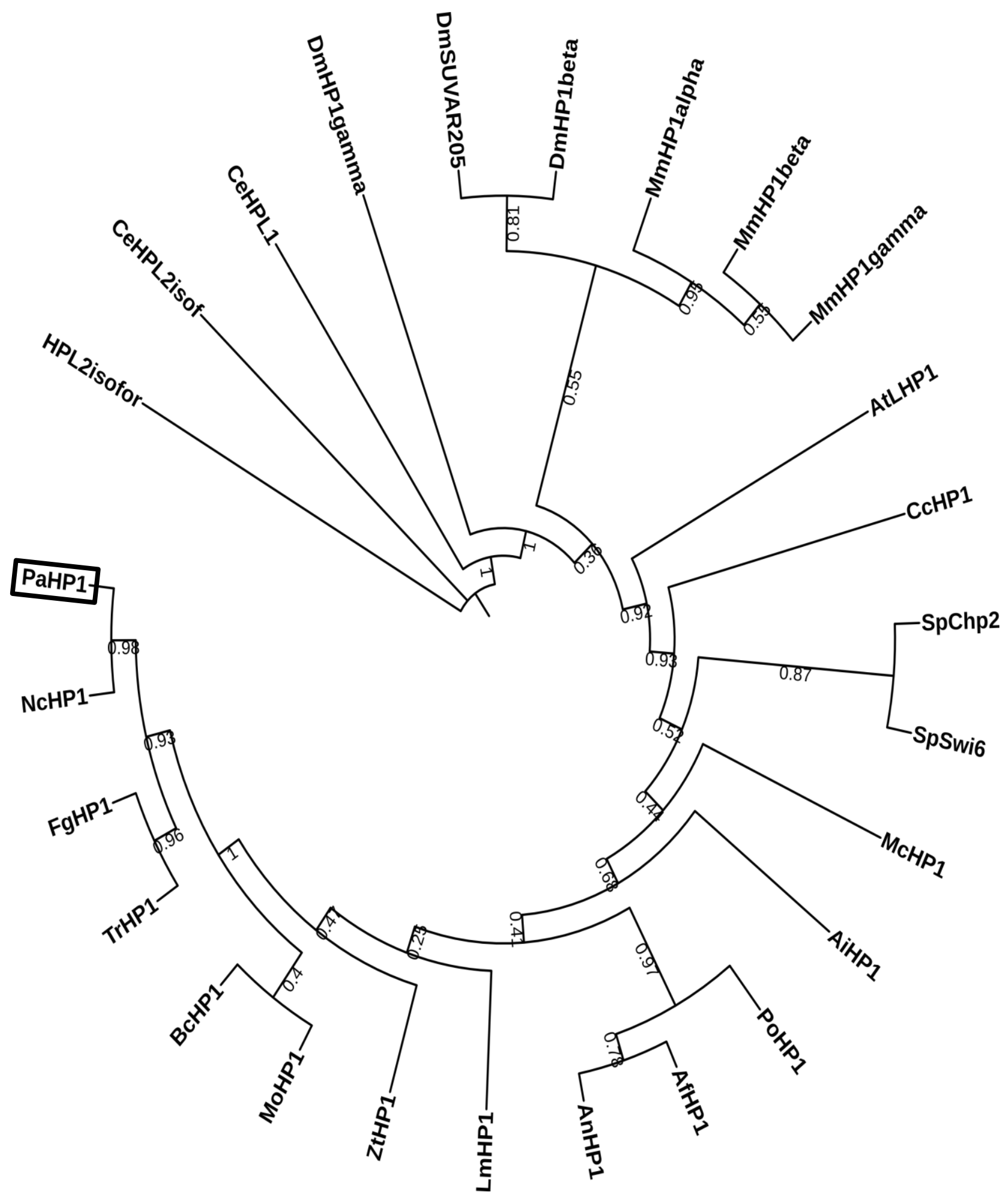


Fig. S12 A
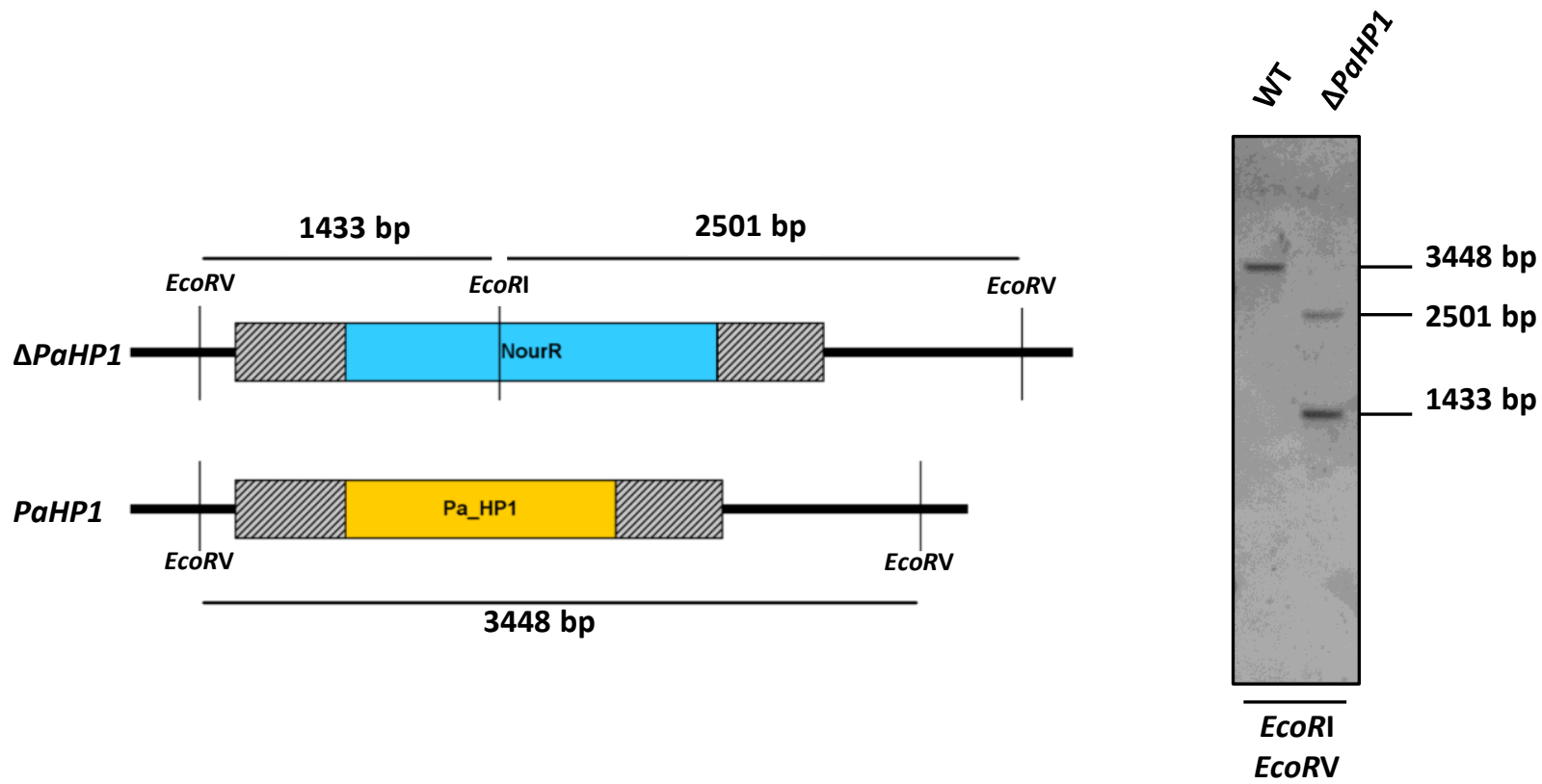

Fig. S12 B
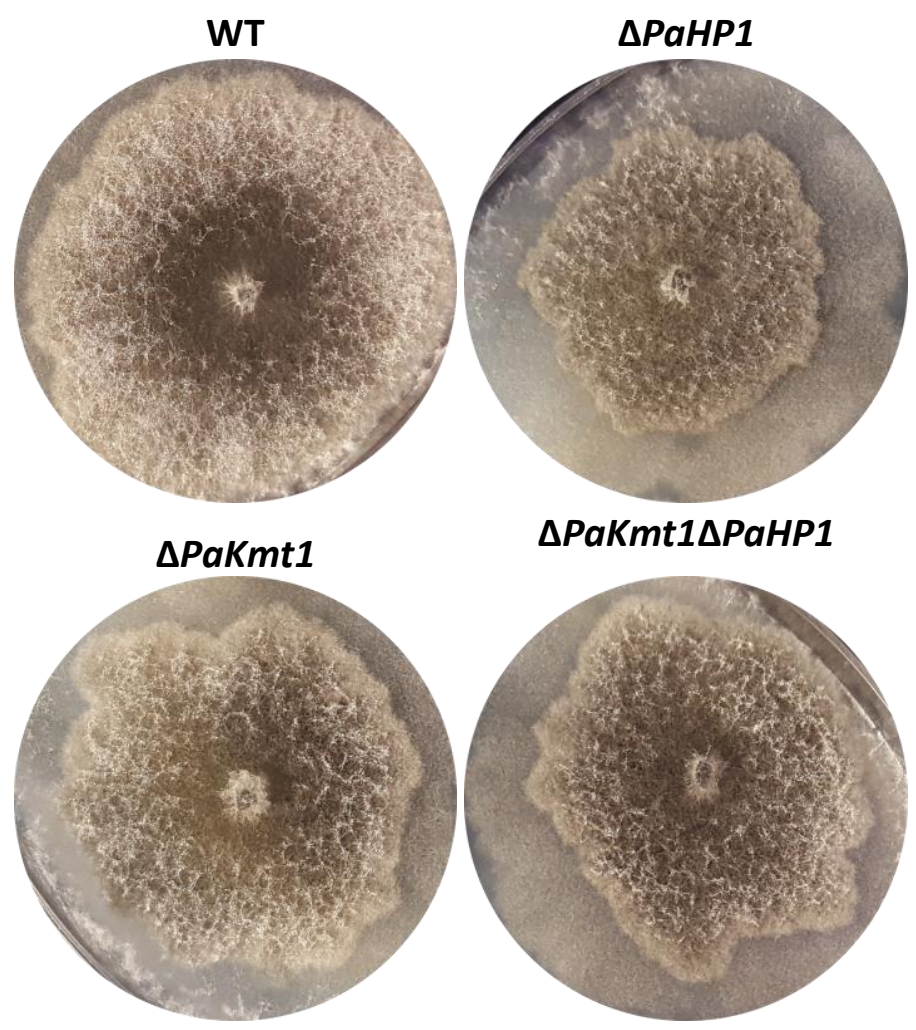

$\triangle P a K m t 1 \triangle P a H P 1$

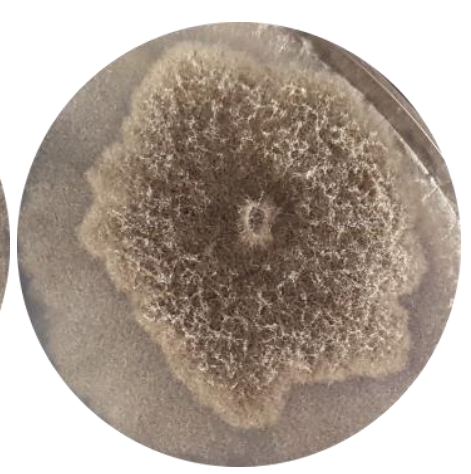


Fig. S12 C

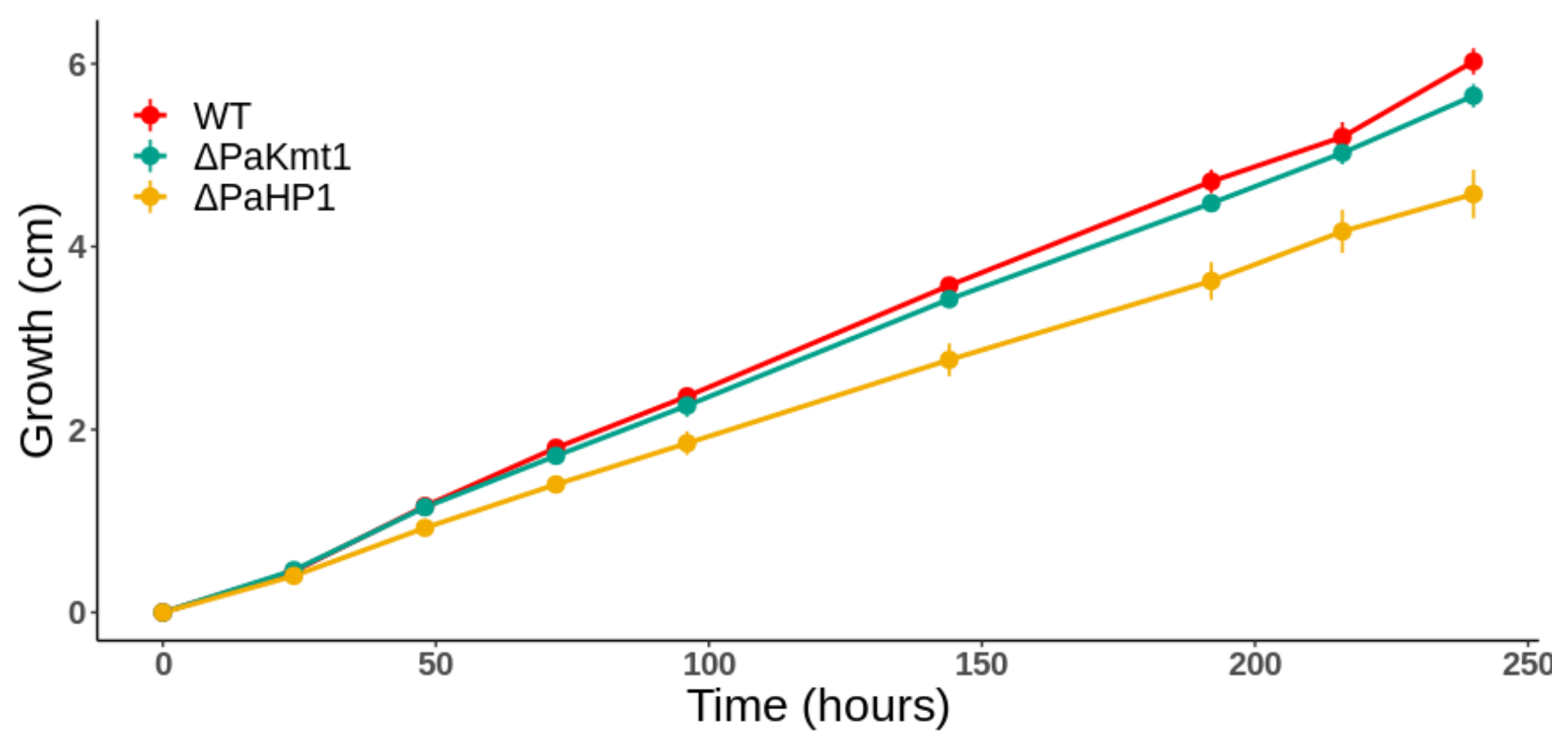


Fig. S12 D
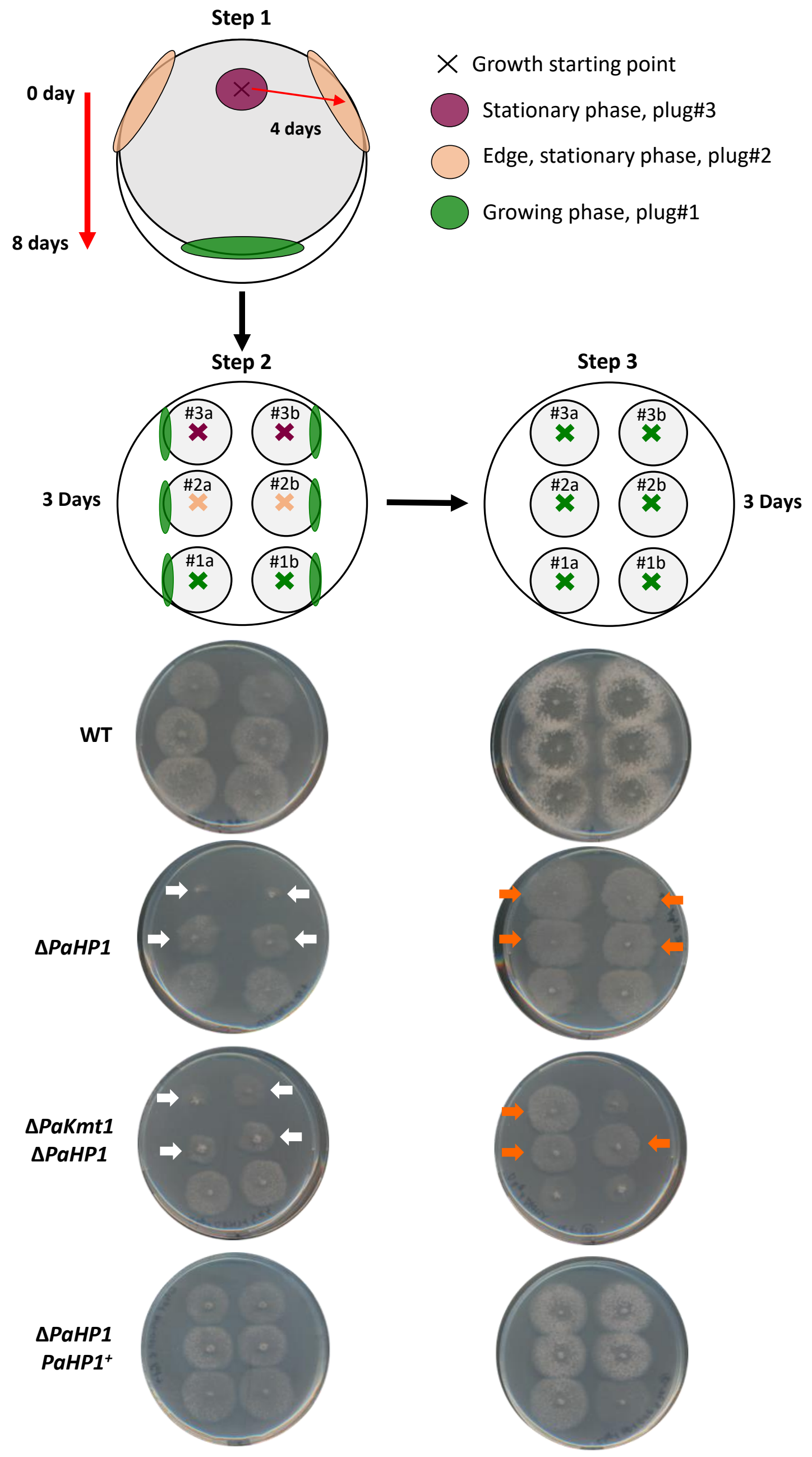
Fig. S12 E

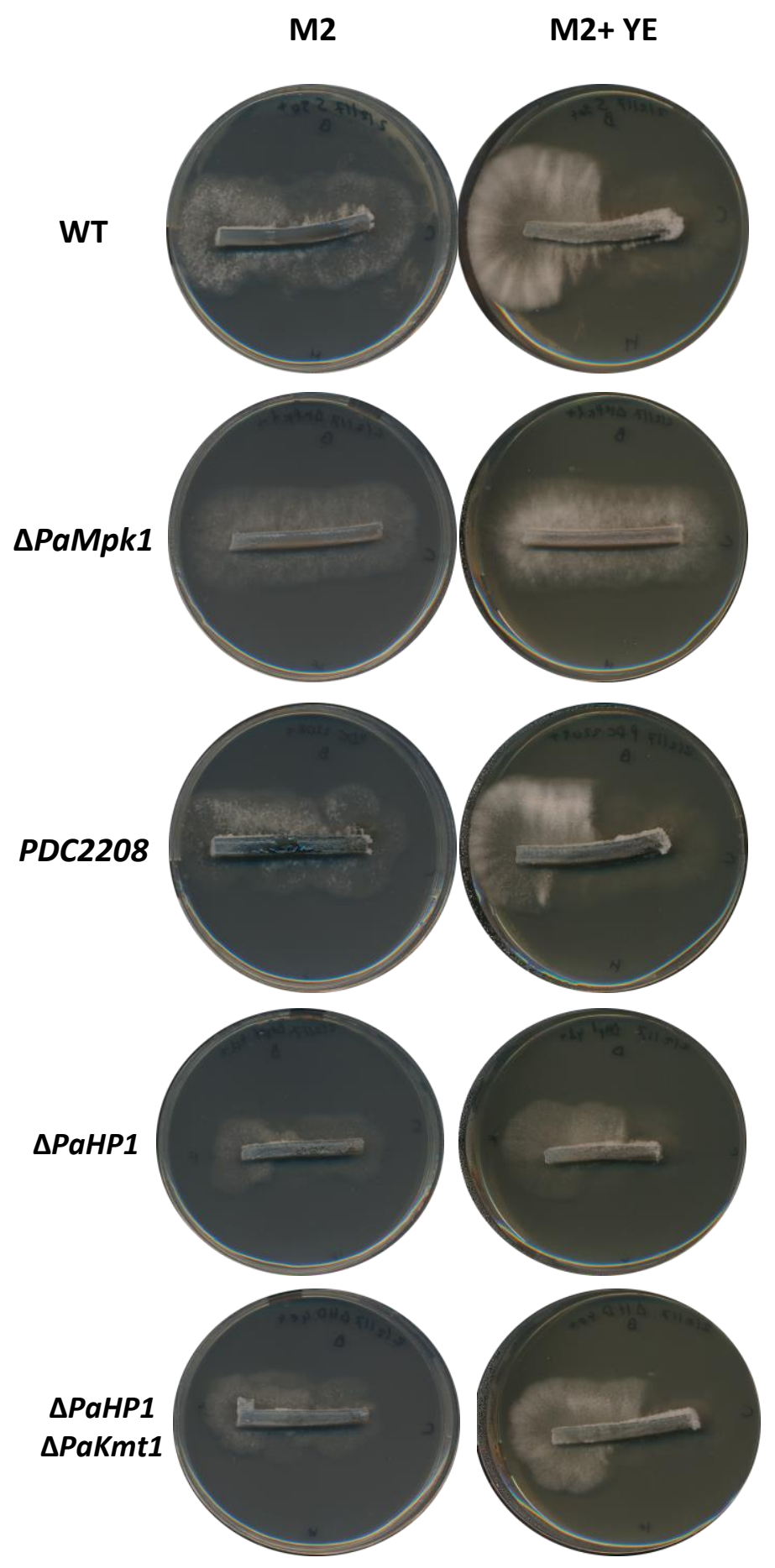


Fig. S13

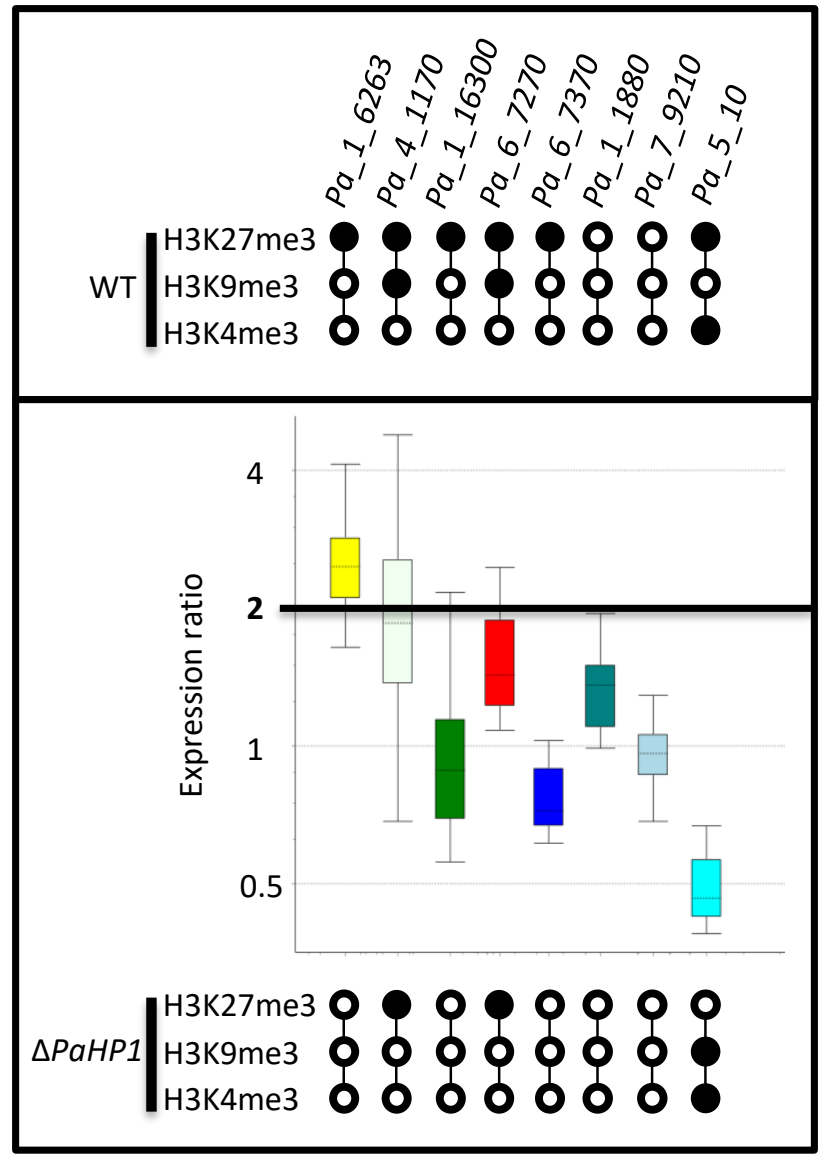


Fig. S14

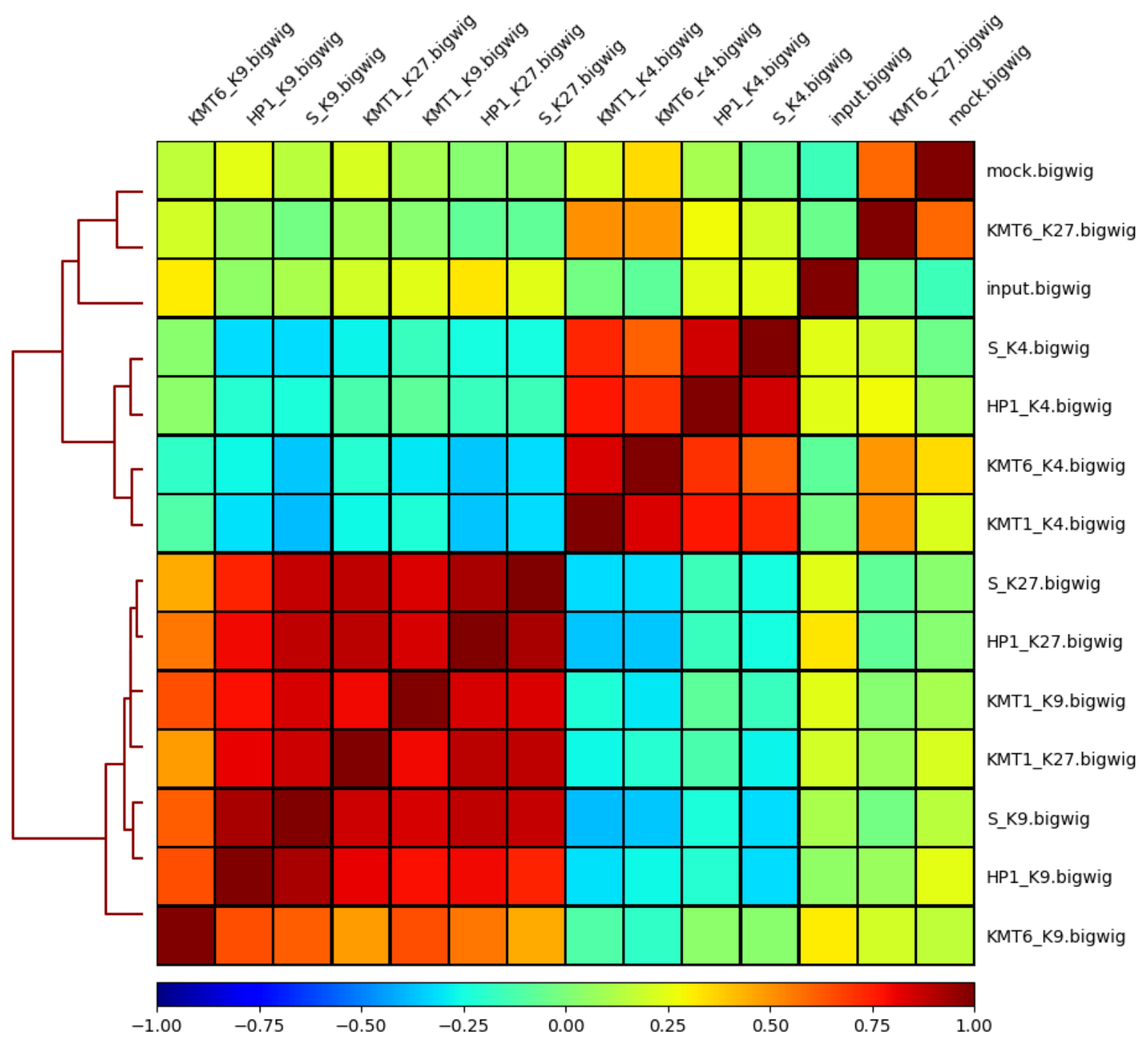




\section{Table S1}

\begin{tabular}{|c|c|c|c|c|c|c|c|c|c|c|}
\hline & & \multicolumn{2}{|c|}{ Genome occupancy } & \multicolumn{2}{|c|}{ Peaks detected } & \multicolumn{2}{|c|}{ Peaks size } & \multicolumn{3}{|c|}{ Marked features } \\
\hline \multirow{4}{*}{ нзК 4mе3 } & WT & 3,84 & 10,98 & 3050,00 & 100,00 & 1259,52 & 100,00 & 3503,00 & 63,00 & 49,00 \\
\hline & $\triangle P a H P 1$ & 3,66 & 10,47 & 3138,00 & 102,89 & 1167,59 & 92,70 & 3587,00 & 64,00 & 52,00 \\
\hline & $\triangle$ PaKmt1 & 4,15 & 11,85 & 3307,00 & 108,43 & 1253,66 & 99,53 & 3793,00 & 67,00 & 58,00 \\
\hline & $\triangle$ РаKMT6 & 4,19 & 11,98 & 3433,00 & 112,56 & 1220,96 & 96,94 & 3983,00 & 85,00 & 69,00 \\
\hline \multirow{2}{*}{ нзкямез } & $\triangle$ PaKmt1 & - & - & - & - & - & - & - & - & - \\
\hline & $\triangle$ РаКMT6 & 0,06 & 0,18 & 85,00 & 7,80 & 730,72 & 77,32 & 2,00 & 129,00 & 4,00 \\
\hline \multirow{3}{*}{ H3К27me3 } & WT & 6,75 & 19,28 & 1941,00 & 100,00 & 3476,75 & 100,00 & 2992,00 & 1042,00 & 307,00 \\
\hline & $\triangle P a H P 1$ & 1,80 & 5,15 & 1443,00 & 74,34 & 1250,34 & 35,96 & 1027,00 & 551,00 & 239,00 \\
\hline & $\triangle P a K m t 1$ & 1,56 & 4,45 & 1326,00 & 68,32 & 1174,28 & 33,78 & 1003,00 & 538,00 & 259,00 \\
\hline
\end{tabular}


bioRxiv preprint doi: https://doi.org/10.1101/2020.08.21.261065; this version posted August 21, 2020. The copyright holder for this preprint (which was not certified by peer review) is the author/funder. All rights reserved. No reuse allowed without permission.

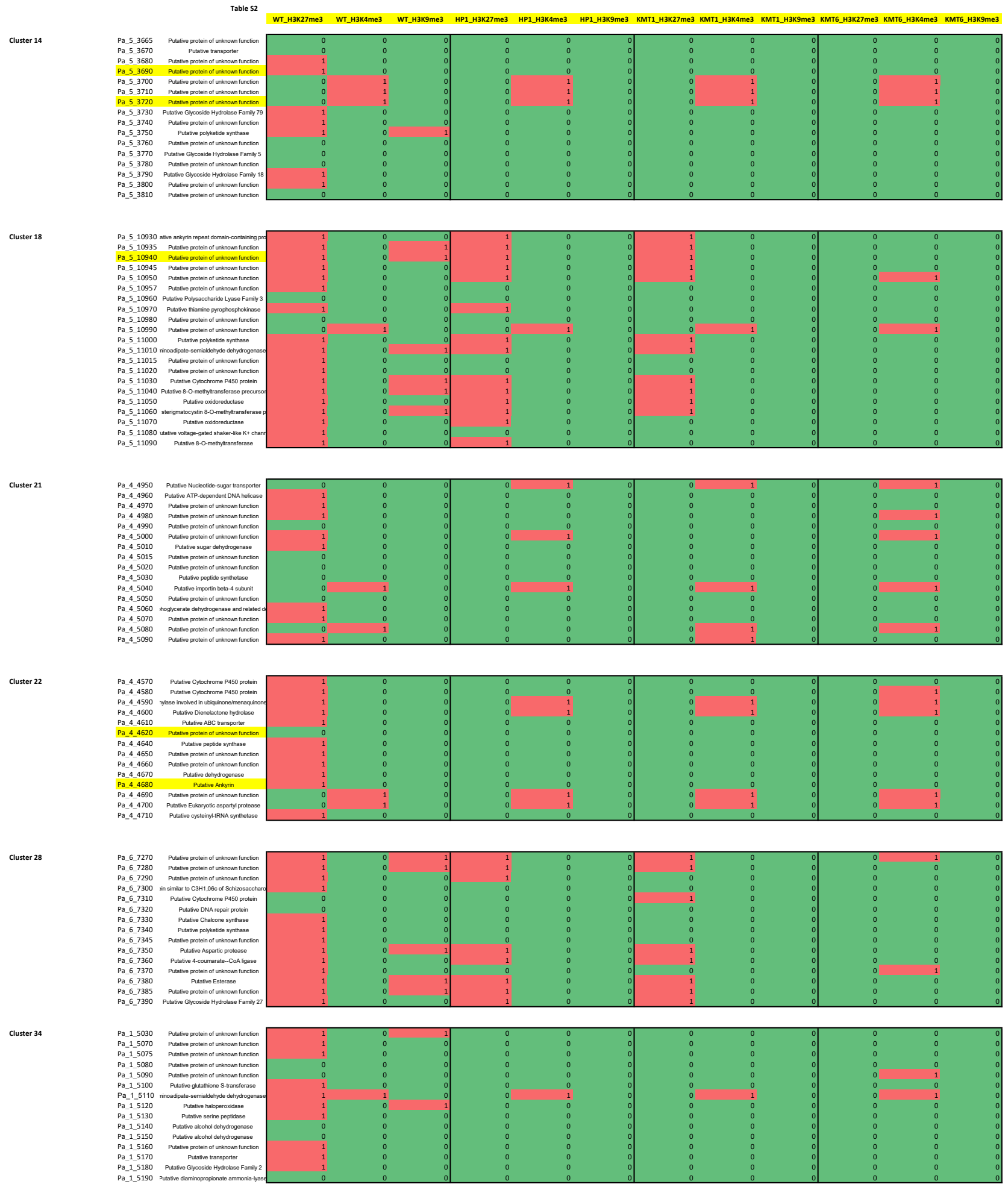


bioRxiv preprint doi: https://doi.org/10.1101/2020.08.21.261065; this version posted August 21, 2020. The copyright holder for this preprint (which was not certified by peer review) is the author/funder. All rights reserved. No reuse allowed without permission.

\section{Table S3}

replicate number

1-1

$1-2$

1-3

1-4

1-5

2-1

$2-2$

2-3

2-4

2-5

3-1

3-2

3-3

3-4

3-5

4-1

4-2

4-3

4-4

4-5
P. anserina strain wild-type

wild-type

wild-type

wild-type

wild-type

$\Delta K m+1$

$\Delta K m+1$

$\Delta K m+1$

$\Delta K m+1$

$\Delta K m+1$

$\Delta K m+6$

$\Delta K m t 6$

$\Delta K m+6$

$\Delta K m+6$

$\Delta K m+6$

$\triangle H p 1$

$\triangle H p 1$

$\triangle H p 1$

$\triangle H p 1$

$\Delta H p 1$ mating type

mat+

mat+

mat+

mat+

mat+

mat+

mat+

mat+

mat+

mat+

mat+

mat+

mat+

mat+

mat+

mat+

mat+

mat+

mat+

mat+ culture condition

$60 \mathrm{~h}, 27^{\circ} \mathrm{C}$, liquid medium as for ChIPseq, see

M \& M for details 
bioRxiv preprint doi: https://doi.org/10.1101/2020.08.21.261065; this version posted August 21, 2020. The copyright holder for this preprint (which was not certified by peer review) is the author/funder. All rights reserved. No reuse allowed without permission.

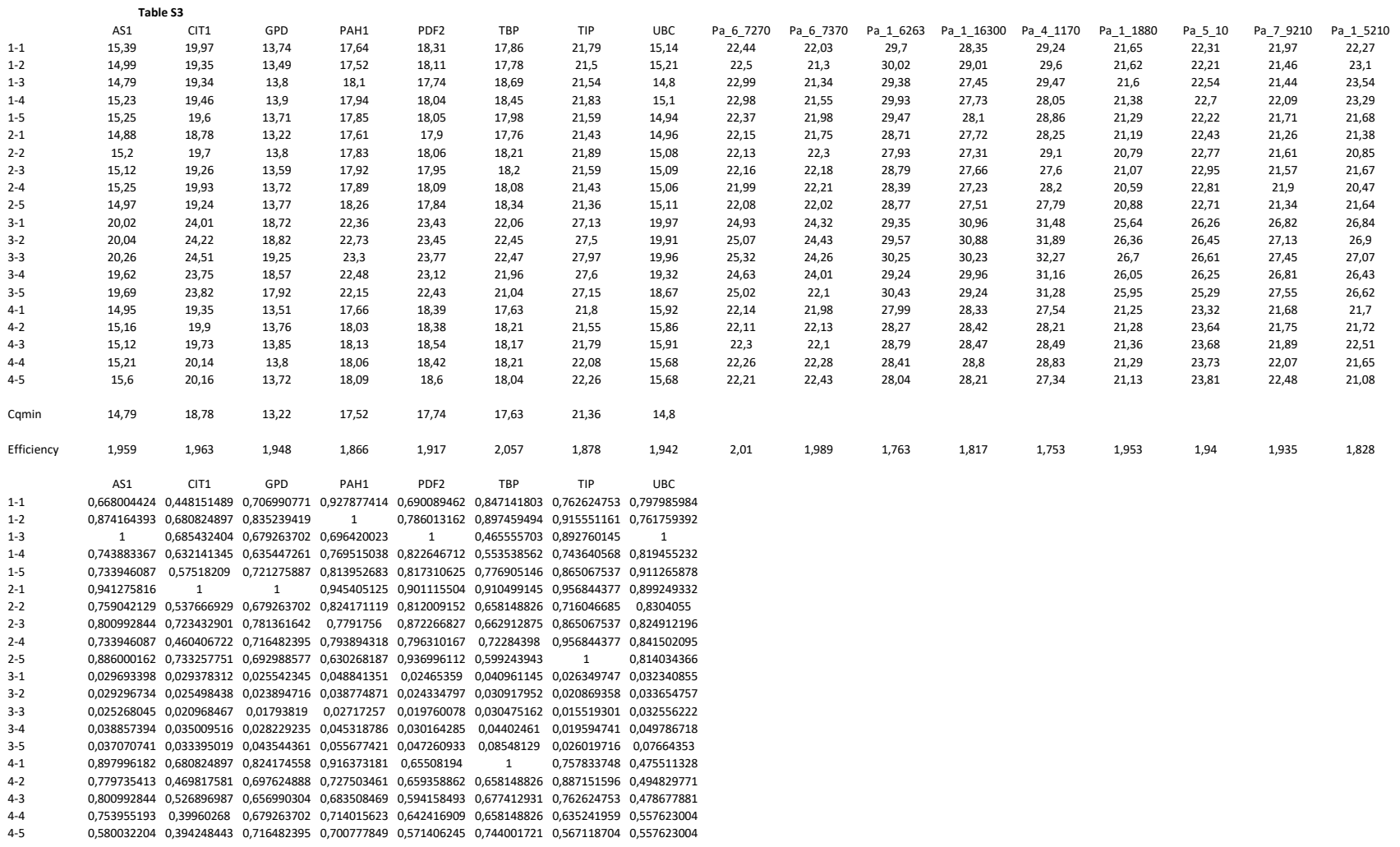
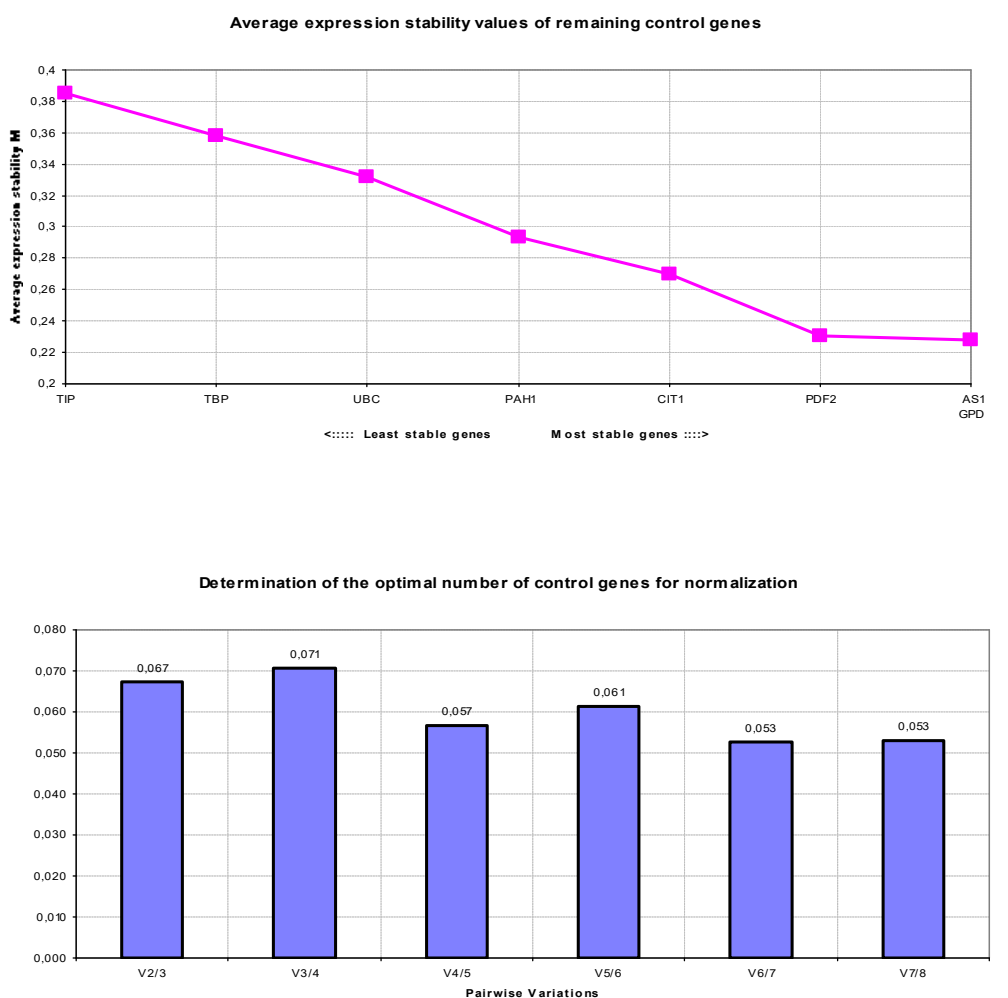
Table S3

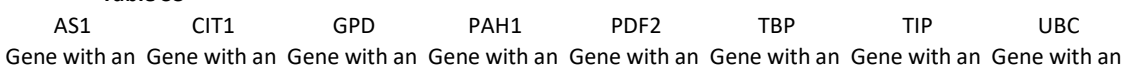

Pa_6_7270

Pa_6 7370 Pa_16263 Pa 116300

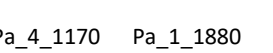

$\underset{N / A}{\text { N } 1 / A 80}$

Pa_7_9210 Pa_10 10 5210 N/A Gene with an Gene with an Gene with an

N/A
N/A
38,78
N/A

N/A $\quad$ N/A

N/A N/A

N/A

36,27

36,84

34,94

N/A

$\mathrm{N} / \mathrm{A}$

36,14

$33,85 \quad N / A$

$1-4$

39,25

$\begin{array}{lll}35,55 & \mathrm{~N} / \mathrm{A} & 34,19 \\ 37,18 & 33,53 & 33,53\end{array}$

$2-2$

$\mathrm{N} / \mathrm{A}$

33,14

37,23

35,01

34,57

33,96

33,57

32,74

30,21
31,31

32,11

32,37

$\begin{array}{cccc}35,43 & 37,18 & 33,53 & 33,53 \\ 35,32 & \mathrm{~N} / \mathrm{A} & \mathrm{N} / \mathrm{A} & \mathrm{N} / \mathrm{A}\end{array}$

$\begin{array}{lllll}\mathrm{N} / \mathrm{A} & \mathrm{N} / \mathrm{A} & 35,28 & \mathrm{~N} / \mathrm{A} & \mathrm{N} / \mathrm{A}\end{array}$

$\begin{array}{lllll}38,68 & 32,77 & 33,69 & 31,87 & 32,2\end{array}$

$\begin{array}{lllll}38,54 & 34,49 & 33,96 & 32,35 & 34,57\end{array}$

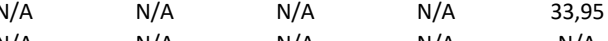

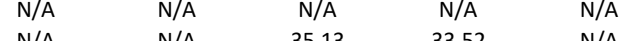

$\begin{array}{lllll}N / A & N / A & 35,13 & 33,52 & N / A\end{array}$

$\begin{array}{lcccc}\text { N/A } & \text { N/A } & \text { N/A } & 34,16 & \text { N/A } \\ \text { N/A } & 34,47 & 34,69 & 32,54 & \text { N/A }\end{array}$

$\begin{array}{ccccc}\mathrm{N} / \mathrm{A} & 34,47 & 34,69 & 32,54 & \mathrm{~N} / \mathrm{A} \\ 40,01 & 33,75 & 34,26 & 32,77 & 33,23\end{array}$

$\begin{array}{lllll}37,88 & 31,96 & 31,6 & 30,19 & 31,17\end{array}$

$\begin{array}{lllll}38,04 & 33,74 & 33,14 & 30,89 & 33,32\end{array}$ 
Table S4 A

\begin{tabular}{|c|c|c|}
\hline Experiments & Name & Sequence $5^{\prime}>3^{\prime}$ \\
\hline \multirow{2}{*}{ PaKmt1 RT-PCR } & 5s-DIM5 & CCCAGTCGAACAACCTCACCAAC \\
\hline & 3s-DIM5 & CCCTGTCAAGCCAGCAGTTTTG \\
\hline \multirow{6}{*}{$\triangle P a H P 1$ allele } & HP1L-2_MkF & CCAAACAAACCACCCCTCGTCGGTTCAGGGCAGGGTCGTTAAATAG \\
\hline & HP1L-3_MkR & GCCATCCCTCTGCTCGAAAACATCGAACTGGATCTCAACAGCGGTAAG \\
\hline & HP1L-1F & САСТССТCGССТСАТСTTCC \\
\hline & Mk_HP1L-2R & CTATTTAACGACCCTGCCCTGAACCGACGAGGGGTGGTTTGTTTGG \\
\hline & Mk_HP1L-3F & CTTACCGCTGTTGAGATCCAGTTCGATGTTTTCGAGCAGAGGGATGGC \\
\hline & HP1L-4R & AGGTTGGCAAATGTGGGAGC \\
\hline \multirow{6}{*}{$\Delta$ PaKmt1 allele } & DIM5-1F & TTTGTCCTTGTTTTTGGGTATGC \\
\hline & Mk_DIM5L-2R & CTATTTAACGACCCTGCCCTGAACCGTTCGTTGACGATGGTGATGG \\
\hline & Mk_DIM5L-3F & CTTACCGCTGTTGAGATCCAGTTCGATGTGGATGAACAAGAGAAACAAACG \\
\hline & DIM5-4R & CTCACAACAACAAGACTCCAACG \\
\hline & DIM5L-2_MkF & CCATCACCATCGTCAACGAAGTTCAGGGCAGGGTCGTTAAATAG \\
\hline & DIM5L-3_MkR & CGTTTGTTTCTCTTGTTCATCCACATCGAACTGGATCTCAACAGCGGTAAG \\
\hline \multirow{6}{*}{$\triangle$ PaKmt6 allele } & KMT6-1F & ACACCGCGAGTATTGATAAACC \\
\hline & Mk_KMT6-2R & CTATTTAACGACCCTGCCCTGAACCGGTCAAATCCACCACAАTTCTCC \\
\hline & Mk_KMT6-3F & CTTACCGCTGTTGAGATCCAGTTCGATGACGGAATTGCTTGACACTAAGG \\
\hline & KMT6-4R & GAGGTGATAGGTGACGATGAGG \\
\hline & KMT6-2_MkF & GGAGAATTGTGGTGGATTTGACCGGTTCAGGGCAGGGTCGTTAAATAG \\
\hline & KMT6-2_MkR & CCTTAGTGTCAAGCAATTCCGTCATCGAACTGGATCTCAACAGCGGTAAG \\
\hline \multirow{5}{*}{$\begin{array}{c}\text { PaHP1-GFP-HA } \\
\text { allele }\end{array}$} & FC3-BamH1 & AAGGATCCGGAGATGCTTGTCCGTCCCTCC \\
\hline & FC4-GFP & GCCCTTGCTCACCATGGAGTTGGCAGGGGGTGAGTCG \\
\hline & FC5-HP1 & CССССTGCСАACTCCATGGTGAGCAAGGGCGAGGAGC \\
\hline & FC6-HindIII & TTAAGCTTCTTGTACAGCTCGTCCATGCCGAGAGTGAT \\
\hline & FC21-BamH1 & AAGGATCCATGCCACCAGGTATGCTTCC \\
\hline \multirow{5}{*}{$\begin{array}{c}\text { PaKmt1-mCH-HA } \\
\text { allele }\end{array}$} & Dim5mChFBamH1 & AAGGATCCGAGCCTTGAGGATGTCTTTAGC \\
\hline & $\operatorname{Dim} 5 \mathrm{mChR}$ & TTGATGATGGCCATCCATAAGAACCCCCTACACTTC \\
\hline & mChDim5F & AGGGGGTTCTTATGGATGGCCATCATCAAGGAGTTC \\
\hline & mChDim5RHindIII & TTAAGCTTCTTGTACAGCTCGTCCATGC \\
\hline & FC22-BamH1 & AAGGATCCATGGAGGAGGCAATGAAACAGC \\
\hline \multirow{4}{*}{$\begin{array}{c}\text { PaKmt6-GFP-HA } \\
\text { allele }\end{array}$} & FC73-GA1KMT6 & AAAGCTGGAGCTCCACCGCGGTGGCGAATGGCATGTATGGTCTG \\
\hline & FC74-GA1KMT6 & CСTTGCTCACСАТСТССТTСТСАТСССТАТАТСТС \\
\hline & FC75-GA1GFP & GGATGAGAAGGAGATGGTGAGCAAGGGCGAG \\
\hline & FC76-GA1GFP & GATATCGAATTCCTGCAGCCCGGGGCCCTTGTACAGCTCGTCCATGC \\
\hline \multirow{2}{*}{ PaKmt6-HA allele } & FC72-BamH1 & TTGGATCCCTCCTTCTCATCССTATATCTCC \\
\hline & FC65-Not1 & TTGCGGCCGCGAATGGCATGTATGGTCTGTGC \\
\hline
\end{tabular}


Table S4 B

\begin{tabular}{|c|c|c|c|c|c|}
\hline $\begin{array}{c}\text { Type } \\
\text { of } \\
\text { gene }^{a}\end{array}$ & $\begin{array}{c}\text { Gene } \\
\text { number }\end{array}$ & $\begin{array}{c}\text { Gene } \\
\text { name or } \\
\text { function }\end{array}$ & $\begin{array}{l}\text { Primer } \\
\text { name }\end{array}$ & Primer sequence $5^{\prime}>3^{\prime} b$ & $\begin{array}{l}\text { Amplicon } \\
\text { size }\end{array}$ \\
\hline \multirow[t]{2}{*}{ HKG } & $P a \_11200$ & Actin & FC77-Actin & CTCCCATCAACCCCAAGAGC & $86 \mathrm{bp}$ \\
\hline & & & FC78-Actin & GATGGAGACGTAGAAGGCGG & \\
\hline \multirow[t]{2}{*}{$\mathrm{HKG}$} & $P a 580$ & 80 & FC125-580 & AGCTACTGTTGTCGTGCTGG & $54 \mathrm{bp}$ \\
\hline & & & FC126-580 & TCGATGAAGAATACGGCGGC & \\
\hline \multirow[t]{2}{*}{ HKG } & $P a \_1 \_16650$ & AS1 & AS1f & CAACATGGCTGACGAATAC/AACGC & 115 bp \\
\hline & & & AS1r & GGAGGTCAGGTCAAGGAGA/GCATC & \\
\hline \multirow[t]{2}{*}{$\mathrm{HKG}$} & $\mathrm{Pa} 3 \quad 6780$ & CIT1 & ClT1f & CTCCTCCAAGACCCAG/ACCCTC & $100 \mathrm{bp}$ \\
\hline & & & CIT1r & GACCTTGGAGCCATGCTCC/TTTC & \\
\hline \multirow[t]{2}{*}{$\mathrm{HKG}$} & $P a \_3 \_5110$ & $G P D$ & GPDf & CATTGAGCCCAAGTACGCT/GAG & $113 \mathrm{bp}$ \\
\hline & & & GPDr & GTCGCGCTCAGTGTAGAACTTGA & \\
\hline \multirow[t]{2}{*}{ HKG } & $\mathrm{Pa} 2$ 2_6460 & PAH1 & PAH1f & GATCTGGTTCCAGAACCG/ACGTG & $247 \mathrm{bp}$ \\
\hline & & & PAH1r & GCAGTTGAGATGATGAATCA/AAAC & \\
\hline \multirow{2}{*}{ HKG } & $\mathrm{Pa} 76690$ & PDF2 & PDFf & GCAGACAGGTTCGAAAAG/ATTG & $294 \mathrm{bp}$ \\
\hline & & & PDFr & CAGATGATCAATGGTT/TCTTGC & \\
\hline \multirow[t]{2}{*}{ HKG } & $\mathrm{Pa}$ _4_8980 & TBP & TBPf & CACACCCACTCTTCA/GAACATT & $106 \mathrm{bp}$ \\
\hline & & & TBPr & ACGCTTGGGGTTGTA/CTCAGC & \\
\hline \multirow[t]{2}{*}{$\mathrm{HKG}$} & $\mathrm{Pa} \mathrm{Z}_{7}{ }_{84990}$ & TIP41 & TIPf & GTTTGCGGAGGTGAAGAAG/AA & $146 \mathrm{bp}$ \\
\hline & & & TIPr & CCGTCTCACCCTCGAGAC & \\
\hline \multirow[t]{2}{*}{$H K G$} & $\mathrm{~Pa} 4$ 4_7790 & $U B C$ & UBCf & GGCCATCCCCATCCATCAAC & $107 \mathrm{bp}$ \\
\hline & & & $\mathrm{UBCr}$ & GGTGATGGTCTTGCCAGTGA/GA & \\
\hline \multirow[t]{2}{*}{ Gol } & $P a \_6 \_7270$ & 7270 & $7270 f 1$ & TAGTCAACATGAAGGGAAGGA & $91 \mathrm{bp}$ \\
\hline & & & 7270r1 & AGTGTAGTAGCGGCTGTCTGT & \\
\hline \multirow[t]{2}{*}{ Gol } & $\mathrm{Pa} 67370$ & 7370 & $7370 f 1$ & CACCATCGCAGACCTCAA/CC & 206 bp \\
\hline & & & 7370r1 & GCTCGGTTTGGTGCCAGG & \\
\hline \multirow[t]{2}{*}{ Gol } & $\mathrm{Pa} 1$ 16263 & 6263 & $6263 f 1$ & TTGTCAACTTTCAGCCT/CC & $227 \mathrm{bp}$ \\
\hline & & & $6263 r 1$ & AAGGGACAAGTAAAACGG/CCT & \\
\hline \multirow[t]{2}{*}{ Gol } & $P a \_1 \_16300$ & 16300 & $16300 f 1$ & CACTTGGACACTCCTG/TCTA & $93 \mathrm{bp}$ \\
\hline & & & 16300r1 & GGTGCCGCTGCCGTCGTA & \\
\hline \multirow[t]{2}{*}{ Gol } & $\mathrm{Pa} 4$ 41170 & 1170 & $1170 f 4$ & TCTGCGAAGGCGAGGAGT & $153 \mathrm{bp}$ \\
\hline & & & $1170 r 4$ & GGGTGATGACGGTGAAAGG & \\
\hline \multirow[t]{2}{*}{ Gol } & $P a \_1 \_1880$ & 1880 & $1880 \mathrm{f} 1$ & AACTATTGCCAGCATTGAGG & $129 \mathrm{bp}$ \\
\hline & & & 1880r1 & AGGTTCTTGCGGTAGGTGA & \\
\hline \multirow[t]{2}{*}{ Gol } & Pa_5_10 & 10 & $10 f 1$ & AGCAGGCCGAGGCATTAA & $91 \mathrm{bp}$ \\
\hline & & & $10 \mathrm{r} 1$ & CCCGCAGCAACATAACCAT & \\
\hline \multirow[t]{2}{*}{ Gol } & $\mathrm{Pa} 79210$ & 9210 & $9210 f 1$ & AATGACGCCGTTTACACCT & $173 \mathrm{bp}$ \\
\hline & & & 9210r1 & GAATCCTCACCACCACTCG & \\
\hline \multirow[t]{2}{*}{ Gol } & $\mathrm{Pa}$ _1_5210 & 5210 & $5210 f 1$ & AGACATTGGCATCAAGAGTAGA & $190 \mathrm{bp}$ \\
\hline & & & 5210r2 & GCAACAGAGGCAGTGGAG & \\
\hline
\end{tabular}

a: HKG: housekeeping gene, used as candidate for reference gene; Gol: gene of interest.

b: slash (/) indicates two consecutive exons. 
bioRxiv preprint doi: https://doi.org/10.1101/2020.08.21.261065; this version posted August 21, 2020. The copyright holder for this preprint (which was not certified by peer review) is the author/funder. All rights reserved. No reuse allowed without permission.

Table S5

SU(VAR)3-9 histone methylase homologues

NcDIM5 XP_957479.2

AnKMT1 CBF88005.1

SpClr4 NP_595186.1

AiKMT1 RPA77146.1

LmKMT1 XP_003835706.1

FgKMT1 PCD21270.1

MoKMT1 XP_003709573.1

ZtKMT1 XP_003856939.1

BcKMT1 XP_001550416.2

EfKMT1 AHF96251.1

AfKMT1 XP_752474.2

CCKMT1 KXN65171.1

MCKMT1 OAD01306.1

PoKMT1 EPS25700.1

TrKMT1 XP_006968782.1

MmSUV39H2 NP_073561.2

MmSUV39H1 NP 035644.1

MmSETDB1 NP_001157113.1

MmGLP NP_001012536.2

MmG9a NP_665829.1

DISUVAR39 NP_524357.2

DmG9a NP_569834.1

DISETDB1 NP_611966.3

CeMET2 NP 498848.4

CESET25 NP_499738.3

AtSUVH4 AAK28969.1

AtSUVR4 OAP05594.1

AtSUVH5 NP_001324386.1

AtSUVH6 NP_850030.1

EZH2 histone methylase homologues

NCSET7 XP_965043.2

AiKMT6 RPA83442.1

LmKMT6 XP_003838658.1

FgKMT6 XP_011316633.1

MoKMT6 XP_003718975.1

ZtKMT6 XP_003853053.1

BcKMT6 XP_001559248.2

PgKMT6 XP_003321175.2

EfKMT6 AHF96252.1

CnEzh2 XP_772865.1

PjKMT6 CCJ29054.1

TrKMT6 ETS00912.1

NMEZH2 NP_031997.2

DMEZ NP_001137932.1

CeMES2 NP_496992.3

AtCLF CAA71599.1

AtSWN NP_567221.1

AtMEA ACN86175.1

Heterochromatin Protein 1 homologues

NcHP1 XP_957632.1

AnHP1 CBF85797.1

SpSwi6 NP_593449.1

SpChp2 NP_596808.1

AiHP1 RPA84120.1

LmHP1 XP_003839601.1

FmHP1 XP_011319962.1

MoHP1 XP_003712460.1

ZtHP1 SMQ49659.1

BcHP1 XP_001554784.1

AfHP1 XP_750646.1

McHP1 OAD06672.1

CcHP1 KXN74185.1

PoHp1 EPS33934.1

TrHP1 ETS00691.1

DmSUVAR205 NP_476755.1

DmHP1beta NP_001162713.1

DmHP1gamma NP_651093.1

CeHPL2isoA NP_001022652.1

CeHPL2isoB NP_001022653.1

CeHPL1 NP_510199.1

MmHP1alpha NP_001345879.1

MmHP1beta NP_031648.1

MmHP1gamma NP_031650.3

AtLHP1 NP_001330017.1 
Table S6

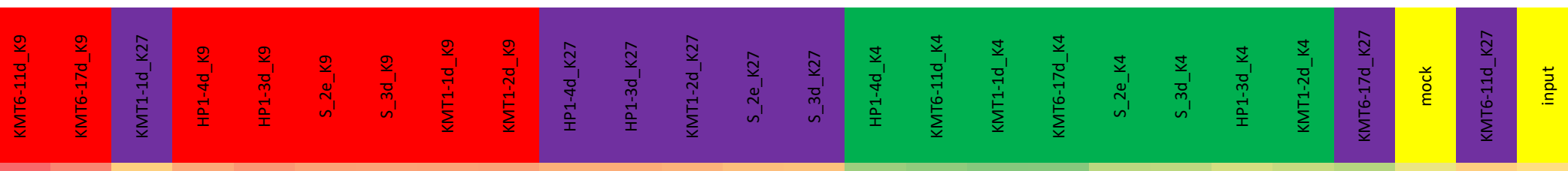

$\begin{array}{llllllllllllllllllllllllllll}1 & 0,834 & 0,378 & 0,617 & 0,731 & 0,656 & 0,651 & 0,66 & 0,672 & 0,575 & 0,592 & 0,553 & 0,491 & 0,469 & -0,165 & -0,21 & -0,253 & -0,249 & -0,047 & -0,041 & 0,051 & -0,006 & -0,069 & 0,138 & 0,395 & 0,294\end{array}$ $\begin{array}{lllllllllllllllllllllllllllllllll}0,834 & 1 & 0,315 & 0,48 & 0,555 & 0,51 & 0,523 & 0,569 & 0,576 & 0,481 & 0,486 & 0,455 & 0,397 & 0,392 & -0,029 & -0,078 & -0,088 & -0,077 & 0,122 & 0,126 & 0,183 & 0,142 & 0,111 & 0,173 & 0,495 & 0,316\end{array}$ $\begin{array}{lllllllllllllllllllllllllllllll}0,378 & 0,315 & 1 & 0,767 & 0,667 & 0,745 & 0,736 & 0,663 & 0,688 & 0,717 & 0,697 & 0,708 & 0,73 & 0,726 & 0,005 & -0,035 & -0,024 & -0,021 & -0,281 & -0,288 & -0,148 & -0,229 & 0,161 & 0,415 & 0,324 & 0,013\end{array}$ $\begin{array}{lllllllllllllllllllllllllllllllllll}0,617 & 0,48 & 0,767 & 1 & 0,843 & 0,867 & 0,83 & 0,703 & 0,721 & 0,701 & 0,681 & 0,667 & 0,677 & 0,651 & -0,116 & -0,149 & -0,107 & -0,131 & -0,303 & -0,315 & -0,205 & -0,283 & 0,092 & 0,355 & 0,237 & -0,076\end{array}$ $\begin{array}{llllllllllllllllllllllllllllllllll}0,731 & 0,555 & 0,667 & 0,843 & 1 & 0,92 & 0,919 & 0,758 & 0,793 & 0,84 & 0,847 & 0,824 & 0,782 & 0,761 & -0,294 & -0,333 & -0,38 & -0,364 & -0,316 & -0,3 & -0,157 & -0,248 & -0,168 & 0,128 & 0,196 & 0,174\end{array}$ $\begin{array}{llllllllllllllllllllllllllllllll}0,656 & 0,51 & 0,745 & 0,867 & 0,92 & 1 & 0,968 & 0,823 & 0,848 & 0,864 & 0,848 & 0,85 & 0,871 & 0,845 & -0,272 & -0,326 & -0,323 & -0,338 & -0,323 & -0,324 & -0,182 & -0,271 & -0,124 & 0,167 & 0,18 & 0,081\end{array}$ $\begin{array}{lllllllllllllllllllllllllllllllll}0,651 & 0,523 & 0,736 & 0,83 & 0,919 & 0,968 & 1 & 0,822 & 0,854 & 0,897 & 0,883 & 0,886 & 0,896 & 0,878 & -0,275 & -0,326 & -0,353 & -0,346 & -0,303 & -0,294 & -0,147 & -0,239 & -0,151 & 0,135 & 0,185 & 0,152\end{array}$ $\begin{array}{llllllllllllllllllllllllllll}0,66 & 0,569 & 0,663 & 0,703 & 0,758 & 0,823 & 0,822 & 1 & 0,964 & 0,821 & 0,809 & 0,813 & 0,822 & 0,795 & -0,195 & -0,265 & -0,252 & -0,288 & -0,15 & -0,167 & 0,005 & -0,073 & -0,133 & 0,1 & 0,279 & 0,235\end{array}$ $\begin{array}{lllllllllllllllllllllllllllllll}0,672 & 0,576 & 0,688 & 0,721 & 0,793 & 0,848 & 0,854 & 0,964 & 1 & 0,858 & 0,851 & 0,852 & 0,852 & 0,829 & -0,195 & -0,26 & -0,268 & -0,289 & -0,166 & -0,175 & -0,003 & -0,087 & -0,134 & 0,106 & 0,28 & 0,259\end{array}$ $\begin{array}{lllllllllllllllllllllllllllllll}0,575 & 0,481 & 0,717 & 0,701 & 0,84 & 0,864 & 0,897 & 0,821 & 0,858 & 1 & 0,941 & 0,945 & 0,916 & 0,911 & -0,266 & -0,315 & -0,379 & -0,348 & -0,255 & -0,236 & -0,063 & -0,156 & -0,198 & 0,041 & 0,202 & 0,306\end{array}$ $\begin{array}{lllllllllllllllllllllllllllllll}0,592 & 0,486 & 0,697 & 0,681 & 0,847 & 0,848 & 0,883 & 0,809 & 0,851 & 0,941 & 1 & 0,945 & 0,902 & 0,904 & -0,276 & -0,314 & -0,392 & -0,356 & -0,258 & -0,235 & -0,043 & -0,143 & -0,215 & 0,02 & 0,215 & 0,342\end{array}$ $\begin{array}{llllllllllllllllllllllllllllllllll}0,553 & 0,455 & 0,708 & 0,667 & 0,824 & 0,85 & 0,886 & 0,813 & 0,852 & 0,945 & 0,945 & 1 & 0,921 & 0,92 & -0,271 & -0,312 & -0,392 & -0,355 & -0,251 & -0,229 & -0,047 & -0,142 & -0,227 & 0,012 & 0,19 & 0,331\end{array}$ $\begin{array}{llllllllllllllllllllllllllllllllll}0,491 & 0,397 & 0,73 & 0,677 & 0,782 & 0,871 & 0,896 & 0,822 & 0,852 & 0,916 & 0,902 & 0,921 & 1 & 0,957 & -0,26 & -0,311 & -0,344 & -0,336 & -0,274 & -0,266 & -0,084 & -0,176 & -0,192 & 0,043 & 0,162 & 0,229\end{array}$ $\begin{array}{llllllllllllllllllllllllllll}0,469 & 0,392 & 0,726 & 0,651 & 0,761 & 0,845 & 0,878 & 0,795 & 0,829 & 0,911 & 0,904 & 0,92 & 0,957 & 1 & -0,223 & -0,269 & -0,319 & -0,3 & -0,238 & -0,223 & -0,031 & -0,128 & -0,181 & 0,027 & 0,176 & 0,251\end{array}$ $\begin{array}{lllllllllllllllllllllllllllllllll}-0,165 & -0,029 & 0,005 & -0,116 & -0,294 & -0,272 & -0,275 & -0,195 & -0,195 & -0,266 & -0,276 & -0,271 & -0,26 & -0,223 & 1 & 0,854 & 0,744 & 0,794 & 0,655 & 0,641 & 0,574 & 0,59 & 0,447 & 0,33 & 0,236 & -0,013\end{array}$

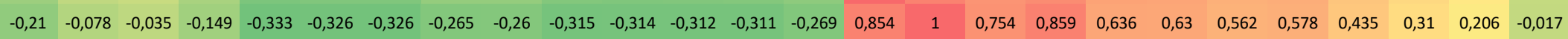

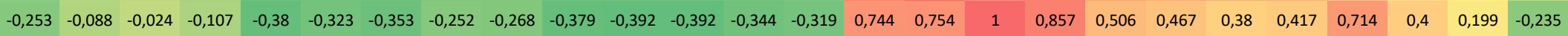
$\begin{array}{lllllllllllllllllllllllllllllll}-0,249 & -0,077 & -0,021 & -0,131 & -0,364 & -0,338 & -0,346 & -0,288 & -0,289 & -0,348 & -0,356 & -0,355 & -0,336 & -0,3 & 0,794 & 0,859 & 0,857 & 1 & 0,568 & 0,55 & 0,465 & 0,492 & 0,617 & 0,395 & 0,253 & -0,1\end{array}$ $\begin{array}{lllllllllllllllllllllllllllllllll}-0,047 & 0,122 & -0,281 & -0,303 & -0,316 & -0,323 & -0,303 & -0,15 & -0,166 & -0,255 & -0,258 & -0,251 & -0,274 & -0,238 & 0,655 & 0,636 & 0,506 & 0,568 & 1 & 0,942 & 0,846 & 0,881 & 0,182 & -0,02 & 0,209 & 0,214\end{array}$ $\begin{array}{lllllllllllllllllllllllllllllllll}-0,041 & 0,126 & -0,288 & -0,315 & -0,3 & -0,324 & -0,294 & -0,167 & -0,175 & -0,236 & -0,235 & -0,229 & -0,266 & -0,223 & 0,641 & 0,63 & 0,467 & 0,55 & 0,942 & 1 & 0,858 & 0,887 & 0,154 & -0,043 & 0,207 & 0,262\end{array}$ $\begin{array}{llllllllllllllllllllllllllllll}0,051 & 0,183 & -0,148 & -0,205 & -0,157 & -0,182 & -0,147 & 0,005 & -0,003 & -0,063 & -0,043 & -0,047 & -0,084 & -0,031 & 0,574 & 0,562 & 0,38 & 0,465 & 0,846 & 0,858 & 1 & 0,898 & 0,079 & -0,079 & 0,256 & 0,35\end{array}$ $\begin{array}{lllllllllllllllllllllllllllllll}-0,006 & 0,142 & -0,229 & -0,283 & -0,248 & -0,271 & -0,239 & -0,073 & -0,087 & -0,156 & -0,143 & -0,142 & -0,176 & -0,128 & 0,59 & 0,578 & 0,417 & 0,492 & 0,881 & 0,887 & 0,898 & 1 & 0,102 & -0,086 & 0,234 & 0,321\end{array}$ $\begin{array}{llllllllllllllllllllllllllllllll}-0,069 & 0,111 & 0,161 & 0,092 & -0,168 & -0,124 & -0,151 & -0,133 & -0,134 & -0,198 & -0,215 & -0,227 & -0,192 & -0,181 & 0,447 & 0,435 & 0,714 & 0,617 & 0,182 & 0,154 & 0,079 & 0,102 & 1 & 0,554 & 0,37 & -0,208\end{array}$ $\begin{array}{llllllllllllllllllllllllllll}0,138 & 0,173 & 0,415 & 0,355 & 0,128 & 0,167 & 0,135 & 0,1 & 0,106 & 0,041 & 0,02 & 0,012 & 0,043 & 0,027 & 0,33 & 0,31 & 0,4 & 0,395 & -0,02 & -0,043 & -0,079 & -0,086 & 0,554 & 1 & 0,449 & -0,149\end{array}$ mock

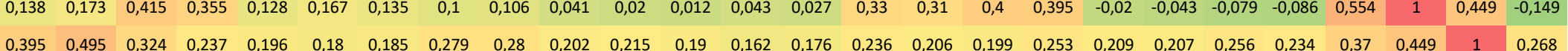
$\begin{array}{clllllllllllllllllllllllllllllllll}\text { input } & 0,294 & 0,316 & 0,013 & -0,076 & 0,174 & 0,081 & 0,152 & 0,235 & 0,259 & 0,306 & 0,342 & 0,331 & 0,229 & 0,251 & -0,013 & -0,017 & -0,235 & -0,1 & 0,214 & 0,262 & 0,35 & 0,321 & -0,208 & -0,149 & 0,268 & 1 & 0\end{array}$ 
Table S7

\begin{tabular}{|c|c|c|c|c|c|c|c|c|c|c|c|c|c|c|}
\hline & 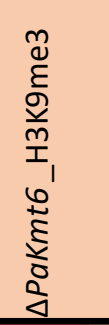 & 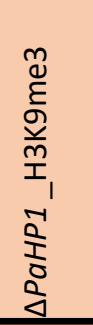 & 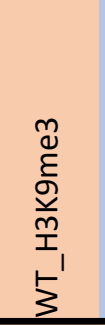 & 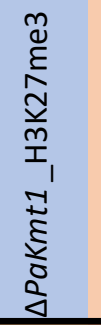 & 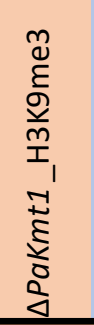 & 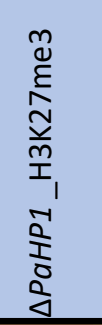 & 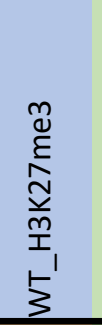 & 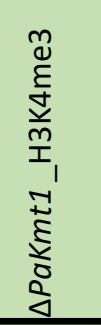 & 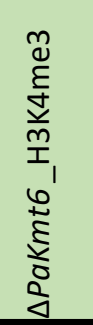 & 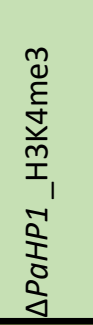 & 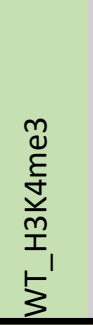 & 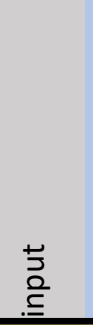 & 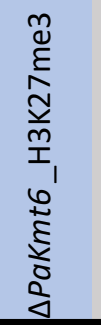 & 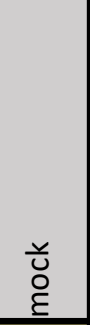 \\
\hline$\triangle$ PaKmt6_H3K9me3 & 1.00 & 0.65 & 0.62 & 0.49 & 0.65 & 0.57 & 0.46 & -0.11 & -0.18 & 0.04 & 0.04 & 0.31 & 0.21 & 0.16 \\
\hline$\triangle P a H P 1 \_H 3 K 9 m e 3$ & 0.65 & 1.00 & 0.92 & 0.82 & 0.78 & 0.80 & 0.75 & -0.31 & -0.26 & -0.21 & -0.32 & 0.05 & 0.08 & 0.26 \\
\hline WT_H3K9me3 & 0.62 & 0.92 & 1.00 & 0.86 & 0.85 & 0.89 & 0.88 & -0.38 & -0.36 & -0.23 & -0.31 & 0.12 & -0.02 & 0.15 \\
\hline$\triangle P a K m t 1 \_H 3 K 27 m e 3$ & 0.49 & 0.82 & 0.86 & 1.00 & 0.80 & 0.89 & 0.88 & -0.26 & -0.21 & -0.12 & -0.27 & 0.21 & 0.09 & 0.22 \\
\hline$\triangle$ PaKmt1_H3K9me3 & 0.65 & 0.78 & 0.85 & 0.80 & 1.00 & 0.85 & 0.84 & -0.22 & -0.29 & -0.08 & -0.16 & 0.25 & 0.03 & 0.10 \\
\hline$\triangle P a H P 1 \_$H3K27me3 & 0.57 & 0.80 & 0.89 & 0.89 & 0.85 & 1.00 & 0.93 & -0.36 & -0.35 & -0.16 & -0.24 & 0.33 & -0.06 & 0.03 \\
\hline WT_H3K27me3 & 0.46 & 0.75 & 0.88 & 0.88 & 0.84 & 0.93 & 1.00 & -0.32 & -0.32 & -0.15 & -0.25 & 0.25 & -0.06 & 0.03 \\
\hline$\triangle$ PaKmt1_H3K4me3 & -0.11 & -0.31 & -0.38 & -0.26 & -0.22 & -0.36 & -0.32 & 1.00 & 0.84 & 0.77 & 0.74 & -0.02 & 0.51 & 0.23 \\
\hline$\triangle$ PaKmt6_H3K4me3 & -0.18 & -0.26 & -0.36 & -0.21 & -0.29 & -0.35 & -0.32 & 0.84 & 1.00 & 0.71 & 0.62 & -0.07 & 0.49 & 0.36 \\
\hline$\triangle P a H P 1$ _H3K4me3 & 0.04 & -0.21 & -0.23 & -0.12 & -0.08 & -0.16 & -0.15 & 0.77 & 0.71 & 1.00 & 0.86 & 0.24 & 0.29 & 0.10 \\
\hline WT_H3K4me3 & 0.04 & -0.32 & -0.31 & -0.27 & -0.16 & -0.24 & -0.25 & 0.74 & 0.62 & 0.86 & 1.00 & 0.25 & 0.21 & -0.03 \\
\hline input & 0.31 & 0.05 & 0.12 & 0.21 & 0.25 & 0.33 & 0.25 & -0.02 & -0.07 & 0.24 & 0.25 & 1.00 & -0.05 & -0.15 \\
\hline$\triangle$ PaKmt6_H3K27me3 & 0.21 & 0.08 & -0.02 & 0.09 & 0.03 & -0.06 & -0.06 & 0.51 & 0.49 & 0.29 & 0.21 & -0.05 & 1.00 & 0.60 \\
\hline mock & 0.16 & 0.26 & 0.15 & 0.22 & 0.10 & 0.03 & 0.03 & 0.23 & 0.36 & 0.10 & -0.03 & -0.15 & 0.60 & 1.00 \\
\hline
\end{tabular}

